\title{
Thermodynamics of the spin-1/2 Heisenberg-Ising chain at high temperatures: a rigorous approach
}

\author{
Frank Göhmann 1 \\ Fakultät für Mathematik und Naturwissenschaften, Bergische Universität Wuppertal, 42097 Wuppertal, Germany. \\ Salvish Goomanee' ${ }^{2}$, Karol K. Kozlowski3 \\ Univ Lyon, ENS de Lyon, Univ Claude Bernard Lyon 1, CNRS, Laboratoire de Physique, F-69342 Lyon, France \\ Junji Suzuki \\ Department of Physics, Shizuoka University, Ohya 836, Shizuoka, Japan.
}

\begin{abstract}
This work develops a rigorous setting allowing one to prove several features related to the behaviour of the Heisenberg-Ising (or XXZ) spin-1/2 chain at finite temperature $T$. Within the quantum inverse scattering method the physically pertinent observables at finite $T$, such as the per-site free energy or the correlation length, have been argued to admit integral representations whose integrands are expressed in terms of solutions to auxiliary non-linear integral equations. The derivation of such representations was based on numerous conjectures: the possibility to exchange the infinite volume and the infinite Trotter number limits, the existence of a real, non-degenerate, maximal in modulus Eigenvalue of the quantum transfer matrix, the existence and uniqueness of solutions to the auxiliary non-linear integral equations, as well as the possibility to take the infinite Trotter number limit on their level. We rigorously prove all these conjectures for temperatures large enough. As a by product of our analysis, we obtain the large- $T$ asymptotic expansion for a subset of sub-dominant Eigenvalues of the quantum transfer matrix and thus of the associated correlation lengths. This result was never obtained previously, not even on heuristic grounds.
\end{abstract}

\section{Introduction and main results}

Quantum statistical mechanics deals with the description of the large volume behaviour of interacting particle systems coupled to a heat bath of temperature $T$. In many situations of interest to physics, the particle degrees of freedom are attached to a finite lattice $\Lambda \subset \mathbb{Z}^{d}$. The interactions between these degrees of freedom are captured by the Hamiltonian $\mathrm{H}$, an operator on the Hilbert space $\mathfrak{h}$ of the model which for a finite lattice $\Lambda$ has a tensor-product structure $\mathfrak{h}=\bigotimes_{a \in \Lambda} \mathfrak{h}_{a}$. Here $\mathfrak{h}_{a}$ plays the role of a local quantum space where the degrees of freedom associated with the lattice site $a \in \Lambda$ evolve. The most basic physically interesting quantity characterising such models is the per-site free energy

$$
-T \lim _{\Lambda \rightarrow \mathbb{Z}^{d}} \frac{1}{|\Lambda|} \ln \operatorname{tr}_{\mathfrak{b}}\left[\mathrm{e}^{-\frac{\mathrm{H}}{T}}\right] .
$$

\footnotetext{
1e-mail: goehmann@uni-wuppertal.de

${ }^{2}$ e-mail: salvish.goomanee@ens-lyon.fr

${ }^{3}$ e-mail: karol.kozlowski@ens-lyon.fr

${ }^{4}$ e-mail: suzuki.junji@shizuoka.ac.jp
} 
In this expression $|\Lambda|$ stands for the number of points in $\Lambda$. It turns out that for quite general Hilbert spaces and Hamiltonians, one may rigorously prove the existence of the above limit, provided that $\Lambda \rightarrow \mathbb{Z}^{d}$ in some appropriate sense [28]. However, obtaining an explicit and thorough characterisation of the limit turned out to be a notoriously hard problem.

So far, rigorous and explicit descriptions of the per-site free energy could only be obtained for one dimensional, $v i z$. when $d=1$, quantum systems and, even in such a simplified setting, only for an extremely limited number of cases. Basically the only rigorous results concern models equivalent to free fermions [24, 34], viz. non-interacting models, and the non-linear Schrödinger model [10]. In both cases the models belong to the class of quantum integrable models for which one may rely on powerful algebraic tools, originating from the theory of quantum groups, to obtain a certain amount of information on the spectra, Eigenvectors and many other observables. Still, the models for which the free energy could be computed rigorously are very specific, even among the quantum integrable models. This implicates, in particular, that the techniques of proofs developed for these models have low chances to be applicable to more complex quantum integrable models. It is the purpose of this paper to develop new strategies and techniques of proofs which will have a large scope of applicability. In this work, we will focus on the XXZ spin-1/2 chain, but more generally, we expect our approach to work for any quantum integrable model associated with a fundamental $R$-matrix.

The XXZ spin-1/2 chain is an archetypical example of a quantum integrable model. It refers to the Hamiltonian operator

$$
\mathrm{H}=J \sum_{a=1}^{L}\left\{\sigma_{a}^{x} \sigma_{a+1}^{x}+\sigma_{a}^{y} \sigma_{a+1}^{y}+\Delta\left(\sigma_{a}^{z} \sigma_{a+1}^{z}+1\right)\right\}-\frac{h}{2} \sum_{a=1}^{L} \sigma_{a}^{z} .
$$

Here $J>0$ represents the so-called exchange interaction, $\Delta \in \mathbb{R}$ is the anisotropy parameter, $h>0$ the external magnetic field and $L \in 2 \mathbb{N}$ corresponds to the number of sites. $H$ acts on the Hilbert space $\mathfrak{h}_{X X Z}=\bigotimes_{a=1}^{L} \mathfrak{h}_{a}$ with $\mathfrak{h}_{a} \simeq \mathbb{C}^{2}, \sigma^{\alpha}, \alpha \in\{x, y, z\}$, are the Pauli matrices, and the operator $\sigma_{a}^{\alpha}$ acts as the Pauli matrix $\sigma^{\alpha}$ on $\mathfrak{h}_{a}$ and as the identity on all the other spaces:

$$
\sigma_{a}^{\alpha}=\underbrace{\mathrm{id} \otimes \cdots \otimes \mathrm{id}}_{a-1} \otimes \sigma^{\alpha} \otimes \underbrace{\mathrm{id} \otimes \cdots \otimes \mathrm{id}}_{L-a} .
$$

Finally, the model is subject to periodic boundary conditions, viz. $\sigma_{L+1}^{\alpha}=\sigma_{1}^{\alpha}$.

The per-site free energy of the XXZ chain is defined as

$$
f=-T \lim _{L \rightarrow+\infty}\left\{\frac{1}{L} \ln \operatorname{tr}_{\mathfrak{h}_{X X Z}}\left[\mathrm{e}^{-\frac{1}{T} \mathrm{H}}\right]\right\} .
$$

As already mentioned, the existence of the limit in (1.4) follows from well-known results in the theory of thermodynamic limits, see e.g. [28]. However, such existence results do not provide one with any direct characterisation of the value of this limit. First approaches based on the integrability of the XXZ chain model and aiming at a characterisation of this limit go back to the works of Gaudin [16] and Takahashi [35]. Those works provided a formal computation of the assumedly dominant contribution, in the large- $L$ limit, to the partition function $\operatorname{tr}_{\mathfrak{h}_{X X Z}}\left[\mathrm{e}^{-\frac{1}{T} \mathrm{H}}\right]$ by generalising the arguments first invoked by Yang and Yang [38] in the context of studying the thermodynamics of another quantum integrable model, the one-dimensional non-linear Schrödinger model [3, 25]. While the approach of Yang and Yang could, in fine, be put on rigorous grounds by Dorlas, Lewis and Pulé [10] for the non-linear Schrödinger model, it appears that doing so in the case of the XXZ spin-chain is hardly possible. Indeed, the proof of [10] invokes, at some stage, the completeness of the Bethe states, a set of eigenstates of an integrable model's Hamiltonian which is parameterised by roots of a coupled system of algebraic equations in many variables, the Bethe Ansatz equations [2]. Whilst completeness of Bethe states could be proven to hold for 
the non-linear Schrödinger model [9], it turns out that completeness issues are, by far more, subtle for the XXZ chain [26]. Gaudin and Takahashi based their calculations on the so-called string hypothesis, assuming that in the large- $L$ limit all solutions of the Bethe Ansatz equations can be classified according to certain patterns of roots in the complex plane, called "string solutions" and discovered by Bethe in his original work [2]. Although the result

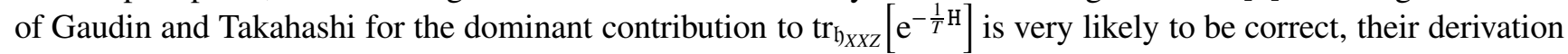
based on the string hypothesis is certainly not. It is well-established for a while [4,7,11,26] that that the so-called "string solutions" do not provide one with a complete set of Eigenvectors for the XXZ chain and, in some cases, do not give rise to solutions of the Bethe equations.

Thus, an alternative approach to the thermodynamics had to be devised. An important step forward in this direction was made by Koma [22,23]. By implementing the Trotter discretisation of the Boltzman statistic operator first proposed by Suzuki [33], and further developed by Suzuki and Inoue [34], Koma proposed a Trotter approximant of the finite- $L$ partition function of the XXX [22] and then of the XXZ [23] chain. By this one means a representation of the type

$$
\operatorname{tr}_{\mathfrak{h}_{X X Z}}\left[\mathrm{e}^{-\frac{1}{T} \mathrm{H}}\right]=\lim _{N \rightarrow+\infty} \operatorname{tr}_{\mathfrak{h}_{\mathfrak{a}}}\left[\mathcal{T}_{N, T}^{L}\right] .
$$

More precisely, Koma rewrote the original finite- $L$ partition function as resulting from taking an infinite Trotter limit $N \rightarrow+\infty$ of the trace of the $L^{\text {th }}$-power of an operator $\mathcal{T}_{N, T}$ acting on an auxiliary Hilbert space $\mathfrak{h}_{\mathfrak{q}}$. Koma's transfer matrix $\mathcal{T}_{N, T}$ corresponds to the transfer matrix of a vertex model related to the product of two Ising models and acts on an auxiliary Hilbert space $\mathfrak{h}_{\mathfrak{q}}$ whose dimension blows up exponentially with $N$. Koma built on the setting of [33,34] so as to establish the validity of the commutativity of the $L \rightarrow+\infty$ and $N \rightarrow+\infty$ limits when substituting (1.5) into (1.4). He also proved that the transfer matrix $\mathcal{T}_{N, T}$ admits a non-degenerate, real, maximal in modulus Eigenvalue $\Lambda_{\max }\left(\mathcal{T}_{N, T}\right)$. This led to the representation

$$
f=-T \lim _{N \rightarrow+\infty}\left\{\ln \left[\Lambda_{\max }\left(\mathcal{T}_{N, T}\right)\right]\right\} .
$$

Within Koma's approach, the Eigenvalue $\Lambda_{\max }\left(\mathcal{T}_{N, T}\right)$ was expressed in terms of a particular solution to the Bethe Ansatz equations. However, Koma only managed to treat rigorously the calculation of the Trotter limit when $\Delta=0$, which is a trivial situation and which was dealt with, by much simpler means, in [34]. For general $\Delta$, Koma did not manage to establish a way of effectively taking the Trotter limit in (1.6). He could only perform a numerical analysis [22] in the case $\Delta=1$. Later, Takahashi [36] proposed a formal scheme for taking the infinite Trotter number limit of the Bethe Ansatz equations obtained by Koma, but that did not lead to any closed formula for $f$, nor was it the result of rigorous handlings.

The above discussion stresses the serious problems arising within Koma's transfer matrix approach relating to taking care, in an efficient way, of the infinite Trotter number limit. Furthermore, Koma's transfer matrix does not appear to exhibit enough algebraic structure so as to allow one for conforming it to a larger class of problems such as the computation of thermal correlation functions, which are the objects of main interest to the physics of the model. For that purpose, as observed in [18], it is more adapted to use a different quantum transfer matrix $t_{\mathfrak{q}}$ directly related to a staggered six-vertex model, whose construction was pioneered in [32] and then further improved in [20], where $t_{\mathrm{q}}$ was introduced as a member of a commuting family of 'column-to-column' transfer matrices. The latter construction has proved to be most convenient in the context of integrable lattice models. Over the years it was used to push rather far, many concrete calculations of various observables associated with the finite temperature XXZ spin-1/2 chain [12,-14, 17,-19]. Those results, which stress the wide scope of applicability of the quantum transfer matrix, constitute an important motivation for putting the handlings based on the use of $t_{q}$ on rigorous grounds.

In the approach based on $t_{q}$, it holds that

$$
\operatorname{tr}_{\mathfrak{h}_{X X Z}}\left[\mathrm{e}^{-\frac{1}{T} \mathrm{H}}\right]=\lim _{N \rightarrow+\infty} \operatorname{tr}_{\mathfrak{h}_{\mathfrak{q}}}\left[\mathrm{t}_{\mathfrak{q}}^{L}\right]
$$


see, e.g. [18] for an exposure thereof in a modern language. The quantum transfer matrix acts on the "quantum' space $\mathfrak{h}_{\mathfrak{q}}=\bigotimes_{a=1}^{2 N} \mathfrak{h}_{a}$, with $\mathfrak{h}_{a} \simeq \mathbb{C}^{2}$. It is defined as the trace over the auxiliary space $\mathfrak{h}_{0}$ of the quantum monodromy matrix

$$
\mathrm{t}_{\mathfrak{q}}=\operatorname{tr}_{\mathfrak{b}_{0}}\left[\mathrm{~T}_{\mathrm{q} ; 0}(0)\right]
$$

where the latter object is defined as an ordered product of R-matrices:

$$
\mathrm{T}_{\mathrm{q} ; 0}(\xi)=\mathrm{R}_{2 N, 0}^{\mathrm{t}_{2 N}}\left(-\frac{\aleph}{N}-\xi\right) \mathrm{R}_{0,2 N-1}\left(\xi-\frac{\aleph}{N}\right) \cdots \mathrm{R}_{2,0}^{\mathrm{t}_{2}}\left(-\frac{\aleph}{N}-\xi\right) \mathrm{R}_{0,1}\left(\xi-\frac{\aleph}{N}\right) \cdot \mathrm{e}^{\frac{h}{2 T} \sigma_{0}^{z}} \quad \text { with } \quad \aleph=\frac{J}{T} \sinh (\eta) .
$$

Above, $\mathrm{R}$ represents the R-matrix of the six-vertex model

$$
\mathrm{R}(\lambda)=\frac{1}{\sinh (\eta)}\left(\begin{array}{cccc}
\sinh (\eta+\lambda) & 0 & 0 & 0 \\
0 & \sinh (\lambda) & \sinh (\eta) & 0 \\
0 & \sinh (\eta) & \sinh (\lambda) & 0 \\
0 & 0 & 0 & \sinh (\eta+\lambda)
\end{array}\right)
$$

while the notation $\mathrm{R}_{a b}$ stands for the embedding of $\mathrm{R}$ into an operator on $\mathfrak{h}_{0} \otimes \mathfrak{h}_{\mathfrak{q}}$ which acts as the identity operator on all spaces $\mathfrak{h}_{k}, k \neq a, b$ and as $\mathrm{R}(\lambda)$ on the spaces $\mathfrak{h}_{a} \otimes \mathfrak{h}_{b}$. Finally, the superscript $\mathfrak{t}_{a}$ stands for the partial transposition with respect to the space $\mathfrak{h}_{a}$.

Here and in the following, for definiteness, we choose the parameterisation

$$
\Delta=\cosh (\eta) \quad \eta=-\mathrm{i} \zeta \quad \text { and } \quad \zeta \in] 0 ; \pi[
$$

corresponding to the regime $-1<\Delta<1$. We shall always assume, without further notice, that $\zeta$ is generic. However, with minor modifications, the whole analysis developed in the following also carries through to the regimes $|\Delta| \geq 1$.

\subsection{Main results of the paper}

On formal grounds, the calculation of the free energy based on the quantum transfer matrix $t_{q}$ follows the same strategy as with Koma's Trotter approximant $\mathcal{T}_{N, L}$. One first assumes the exchangeability of the thermodynamic $(L \rightarrow+\infty)$ and the Trotter $(N \rightarrow+\infty)$ limits. Then, one assumes that $t_{\mathrm{q}}$ admits a maximal in modulus, real non-degenerate Eigenvalue $\widehat{\Lambda}_{\text {max }}$. The two conjectures put together, allow one to write the per-site free energy of the model as

$$
f=-T \lim _{N \rightarrow+\infty}\left\{\ln \left[\widehat{\Lambda}_{\max }\right]\right\} .
$$

On the basis of Bethe Ansatz calculations and a certain amount of numerical input, one then argues an integral representation for $\widehat{\Lambda}_{\max }$ which is given in terms of a solution $\widehat{\mathfrak{A}}$ to an auxiliary non-linear integral equation. By assuming that it is licit to take the infinite Trotter limit formally on the level of this non-linear integral equation, one obtains an integral representation for $f$. This strategy was developed in [5,21]. While all of the above assumptions were supported by thorough numerical calculations, no mathematically rigorous proof was ever given. The main progress achieved in this work consists in establishing rigorously, for $T$ large enough:

i) the exchangeability of the Trotter and infinite volume limits;

ii) the existence of a maximal in modulus Eigenvalue of the quantum transfer matrix which, furthermore, is real and non-degenerate; 
iii) the well-definiteness of the class of non-linear integral equations describing the Eigenvalues of $t_{q}$, as well as the existence and uniqueness of their solutions;

iv) the rigorous identification of the non-linear integral equation describing the dominant Eigenvalue of the quantum transfer matrix.

Points i)-iv), all put together, allow one to establish the below theorem which crowns the efforts of this paper. In order to state the theorem, we shall agree that $\mathcal{D}_{z_{0}, \alpha} \subset \mathbb{C}$ stands for the open disc of radius $\alpha$ centred at $z_{0}, c . f$. (1.20). Also, $\mathcal{B}_{r}$ stands for the space of holomorphic functions on the strip of width $\frac{1}{2} \min (\zeta, \pi-\zeta)$ around $\mathbb{R}$ whose $L^{\infty}$-norm on this strip is bounded by $r, c . f .(1.21$.

Theorem 1.1. There exist $T_{0}>0$ and $\epsilon>0$ such that for any $T>T_{0}$, the per-site free energy of the XXZ chain defined by (1.4) admits the integral representation

$$
-\frac{f}{T}=\frac{h}{2 T}-\frac{2 J}{T} \cos (\zeta)-\oint_{\partial \mathcal{D}_{0, \epsilon}} \frac{\sin (\zeta) \mathcal{L} \mathrm{n}\left[1+\mathrm{e}^{\mathfrak{A}}\right](u)}{\sinh (u-\mathrm{i} \zeta) \sinh (u)} \cdot \frac{\mathrm{d} u}{2 \pi}
$$

in which $\mathfrak{A}$ is the unique solution to the non-linear integral equation on $\mathcal{B}_{\mathrm{c}}$

$$
\mathfrak{A}(\xi)=-\frac{1}{T}\left\{h-\frac{2 J \sin ^{2}(\zeta)}{\sinh (\xi) \sinh (\xi-\mathrm{i} \zeta)}\right\}+\oint_{\partial \mathcal{D}_{0, \epsilon}} \frac{\sin (2 \zeta) \cdot \mathcal{L} \mathrm{n}\left[1+\mathrm{e}^{\mathfrak{A}}\right](u)}{\sinh (\xi-u+\mathrm{i} \zeta) \sinh (\xi-u-\mathrm{i} \zeta)} \cdot \frac{\mathrm{d} u}{2 \pi} .
$$

Here, the logarithm is defined as

$$
\mathcal{L} \mathrm{n}\left[1+\mathrm{e}^{\mathfrak{A}}\right](\xi)=\int_{\epsilon}^{\xi} \frac{\mathfrak{A}^{\prime}(u)}{1+\mathrm{e}^{-\mathfrak{H}(u)}} \cdot \frac{\mathrm{d} u}{2 \mathrm{i} \pi}+\ln \left[1+\mathrm{e}^{\mathfrak{I}(\epsilon)}\right]
$$

in which the integral runs from $\epsilon$ to $\xi \in \partial \mathcal{D}_{0, \epsilon}$ in positive direction along $\partial \mathcal{D}_{0, \epsilon}$ and $\ln$ stands for the principal branch of the logarithm with $\arg \in[-\pi ; \pi[$. The claim of uniqueness and existence of solutions to (1.14) is part of the statement of the theorem.

Finally, the per-site free energy admits the high-T asymptotic expansion:

$$
-\frac{f}{T}=\ln 2-\frac{J \cos (\zeta)}{T}+\mathrm{O}\left(T^{-2}\right) .
$$

The expansion (1.16) was already obtained in the literature, without the rigorous justification, through various methods [6, 27], up to order $\mathrm{O}\left(T^{-5}\right)$ for the XXZ chain in a magnetic field and up to order $\mathrm{O}\left(T^{-100}\right)$ for the XXX chain at zero magnetic field [29]. In fact, taking for granted the conclusions of Theorem 1.1, it is not a problem to build on the non-linear integral equation (1.14) so as to push the expansion (1.16) to very high orders in $T^{-1}$, this with the help of formal computer algebra.

The techniques developed in this work allow one, in fact, to obtain a much larger range of results. Indeed, while the dominant Eigenvalue $\widehat{\Lambda}_{\max }$ of the quantum transfer matrix provides a means to access the per-site free energy through (1.12), the other Eigenvalues $\widehat{\Lambda}_{\text {ex; } k}$ provide an access to the correlation lengths $\xi_{k}$ of the model. These control the speed of the exponential decay of multi-point correlation functions seen as a function of the distances separating the local operators, see e.g. [12,13] for more details. The correlation lengths are defined as

$$
\mathrm{e}^{-\frac{1}{\xi_{k}}}=\lim _{N \rightarrow+\infty}\left\{\frac{\widehat{\Lambda}_{\mathrm{ex} ; k}}{\widehat{\Lambda}_{\max }}\right\} \text {. }
$$


Our analysis allows us to show that, for temperatures large enough, and for a rather large subset of the Eigenvalues $\widehat{\Lambda}_{\text {ex; } k}$ the limit (1.17) exists and is characterised in terms of the unique solution to a non-linear integral equation. See Proposition 5.3. Theorem 5.4 and Proposition 6.2 for more details. These results allow one, in the end, to characterise rigorously the large-temperature behaviour of a certain subset of the correlation lengths.

Theorem 1.2. Fix integers $n_{x}, n_{y}$ and pick some $\varrho>0$ small enough. Let $\left\{y_{a}\right\}_{1}^{n_{y}}$ correspond to a solution to the system

$$
(-1)^{n_{x}-n_{y}+1} \prod_{b=1}^{n_{y}}\left\{\sinh \left(\mathrm{i} \zeta+y_{b}-y_{a}\right)\right\} \cdot\left(\sinh \left(\mathrm{i} \zeta+y_{a}\right)\right)^{n_{x}}=\prod_{b=1}^{n_{y}}\left\{\sinh \left(\mathrm{i} \zeta+y_{a}-y_{b}\right)\right\} \cdot\left(\sinh \left(\mathrm{i} \zeta-y_{a}\right)\right)^{n_{x}}
$$

for $a=1, \ldots, n_{y}$, such that the following three subsidiary constraints are satisfied

1. $y_{a} \neq y_{b} \pm \mathrm{i} \zeta, \quad y_{a} \neq y_{b} \quad \operatorname{modi} \pi \mathbb{Z} \quad$ for $\quad a, b \in \llbracket 1 ; n_{y} \rrbracket$;

2. $\left|(-1)^{n_{x}-n_{y}} \prod_{b=1}^{n_{y}} \frac{\sinh \left(\mathrm{i} \zeta+y_{b}\right)}{\sinh \left(\mathrm{i} \zeta-y_{b}\right)}+1\right|>\varrho$;

3. for $a=1, \ldots, n_{y}$,

$$
y_{a} \in\left\{z \in \mathbb{C}:|\mathfrak{J}(z)| \leq \frac{\pi}{2}, z \notin \mathcal{D}_{ \pm \mathrm{i} \zeta_{\mathrm{m}}, \varrho} \cup\{0\}\right\} \quad \text { with } \quad \zeta_{\mathrm{m}}=\min \{\zeta, \pi-\zeta\} .
$$

Finally, let $h_{1}, \ldots, h_{n_{x}} \in \mathbb{Z}$ be pairwise distinct. Then, there exists a correlation length $\xi_{k}$ whose large-T asymptotics take the form

$$
\mathrm{e}^{-\frac{1}{\xi_{k}}} \cdot \mathrm{e}^{-\frac{f}{T}}=\frac{1}{T^{n_{x}}} \prod_{a=1}^{n_{x}}\left\{\frac{-2 \mathrm{i} J}{\left(2 h_{a}+1+n_{x}-n_{y}\right) \pi-\sum_{a=1}^{n_{y}} \theta_{+}\left(-y_{a}\right)}\right\} \prod_{a=1}^{n_{y}} \frac{\sinh \left(y_{a}-\mathrm{i} \zeta\right)}{\sinh \left(y_{a}\right)}
$$

where $-\mathrm{i} \theta$ is a determination of the logarithm of $\lambda \mapsto \sinh (\mathrm{i} \zeta+\lambda) / \sinh (\mathrm{i} \zeta-\lambda)$ and the + subscript in $\theta_{+}$indicates that one should take the + boundary value if $-y_{a}$ is located on the logarithm's cut.

This theorem thus provides a classification of a subset of the correlation lengths of the spin-1/2 XXZ chain in terms of solutions to the Bethe Ansatz equations (1.18) describing the spectrum of a spin-1 XXZ chain of length $n_{x}, c . f$. [30, 31]. Also, note that the choice of the determination of the logarithm in Theorem 1.2] is irrelevant in that it simply results in shifts, by a global integer, of the integers $h_{1}, \ldots, h_{n_{x}}$.

\subsection{Notations}

- Given $\alpha>0, z_{0} \in \mathbb{C}$, we shall denote

$$
\mathcal{S}_{\alpha}=\{z \in \mathbb{C}:|\mathfrak{I}(z)|<\alpha\} \quad \text { and } \quad \mathcal{D}_{z_{0}, \alpha}=\left\{z \in \mathbb{C}:\left|z-z_{0}\right|<\alpha\right\} .
$$

- Given an open subset $U \subset \mathbb{C}, O(U)$ stands for the ring of holomorphic functions on $U$, and for any function $g$ on $U$, we denote $\|g\|_{L^{\infty}(U)}=$ supess $_{u \in U}|g(u)|$.

- Given $r>0$, set

$$
\mathcal{B}_{r}=\left\{\xi \in O\left(\mathcal{S}_{\zeta_{\mathrm{m}} / 2}\right):\|\xi\|_{L^{\infty}\left(\mathcal{S}_{\zeta_{\mathrm{m}} / 2}\right)} \leq r\right\} \quad \text { with } \quad \zeta_{\mathrm{m}}=\min (\zeta, \pi-\zeta) .
$$


- For integers $a<b, \llbracket a ; b \rrbracket=\{a, a+1, \ldots, b\}$.

- Consider $n$ complex numbers $z_{1}, \ldots, z_{n} \in \mathbb{C}$, distinct or not. If some roots coincide, the total number of roots equal to a given complex number $z$ is called their multiplicity and denoted $k_{z}$. One then defines the set

$$
\left\{z_{a}\right\}_{1}^{n}=\left\{\left(\lambda, k_{\lambda}\right): \lambda \in\left\{z_{1}, \ldots, z_{n}\right\}\right\}
$$

in which $\left\{z_{1}, \ldots, z_{n}\right\}$ stands for the usual set build up from the numbers $z_{1}, \ldots, z_{n}$.

- Given $\Omega$ a finite set and a map $n:(\Omega, x) \rightarrow\left(\mathbb{Z}, n_{x}\right)$, the weighted cardinality $|A|$ of the set $A=\left\{\left(x, n_{x}\right): x \in\right.$ $\Omega\}$ is defined by $|A|=\sum_{x \in \Omega} n_{x}$.

- Given a function $f$ on the set $\Omega$ and $A$ defined as above, we agree upon the shorthand notation

$$
\sum_{\lambda \in A} f(\lambda) \equiv \sum_{x \in \Omega} n_{x} f(x) \quad \text { and } \quad \prod_{\lambda \in A} f(\lambda) \equiv \prod_{x \in \Omega}\{f(x)\}^{n_{x}} .
$$

- Given two sets $A=\left\{\left(x, n_{x}\right): x \in \Omega_{A}\right\}$ and $B=\left\{\left(y, n_{y}\right): x \in \Omega_{B}\right\}$, one defines their algebraic sum $\oplus$ and difference $\ominus$ as

$$
A \oplus B=\left\{\left(x, n_{x}+m_{x}\right): x \in \Omega_{A} \cup \Omega_{B}\right\}, \quad A \ominus B=\left\{\left(x, n_{x}-m_{x}\right): x \in \Omega_{A} \cup \Omega_{B}\right\}
$$

in which one understands that the maps $n$ and $m$ are extended as $n_{x}=0$, resp. $m_{x}=0$, on $\Omega_{B} \backslash \Omega_{A}$, resp. $\Omega_{A} \backslash \Omega_{B}$. Furthermore, if $\Omega_{A}, \Omega_{B}$ are two sets, then $\Omega_{A} \ominus \Omega_{B} \equiv A \ominus B$, where $A, B=\left\{(x, 1): x \in \Omega_{A, B}\right\}$

- These notation allow one to introduce compact notations for sums and products. Given a function $f$ on $\Omega_{A} \cup \Omega_{B}$ parameterising sets $A, B$ as above,our conventions imply

$$
\sum_{\lambda \in A \ominus B} f(\lambda)=\sum_{x \in \Omega_{A}} n_{x} f(x)-\sum_{y \in \Omega_{B}} m_{y} f(y), \quad \prod_{\lambda \in A \ominus B} f(\lambda)=\frac{\prod_{x \in \Omega_{A}}\{f(x)\}^{n_{x}}}{\prod_{y \in \Omega_{B}}\{f(y)\}^{m_{y}}} .
$$

- Given a point $x \in \mathbb{C},\{x\}^{\oplus n}$ denotes the set $\{(x, n)\}$, meaning that one should understand

$$
\sum_{t \in\{x\}^{\oplus n}} f(t) \equiv n f(x) .
$$

- Given an operator $\mathrm{A},\|\mathrm{A}\| \|$ stands for its norm, $\sigma(\mathrm{A})$ for its spectrum and $r_{S}(\mathrm{~A})$ for its spectral radius.

- In stands for the principal branch of the logarithm continued to $\mathbb{R}^{-} \backslash\{0\}$ with the convention $\arg (z) \in[-\pi ; \pi[$.

\subsection{Outline of the paper}

The paper is organised as follows. In Section 2 we develop a setting allowing one to establish, for $T$ large enough, various structural properties of the spectrum of the quantum transfer matrix. In Section 3 we apply these results to the proof of properties i)-ii) stated earlier on. Then, in Section 4, we recall the connection, at finite Trotter number, between the Bethe Ansatz approach to the characterisation of the spectrum of $t_{q}$ and non-linear integral equations. In Section 5 we develop the rigorous treatment, for $T$ large enough, of these non-linear integral equations. In Section 6, we gather all of the previous results so as to establish Theorem 1.1. As a byproduct we also characterise the expression, at high $T$, of the model's correlation lengths. Quite remarkably, these appear to be governed by solutions to the Bethe Ansatz equation for the spin-1 XXZ chain. Finally, in Section 7, we illustrate our analysis by providing numerical results for the solution sets to the Bethe Ansatz equations describing the spectrum of the quantum transfer matrix at finite Trotter number. 


\section{Traces of powers of the quantum transfer matrix at high temperatures}

It is useful to recall that given an elementary matrix $\mathrm{E}^{i k}$ acting on $\mathcal{L}\left(\mathbb{C}^{2}\right), \mathrm{E}_{a}^{i k}$ stands for its canonical embedding into an operator on $\mathfrak{h}_{0} \otimes \mathfrak{h}_{\mathfrak{q}}$ acting non-trivially only on the $a^{\text {th }}$ factor in the tensor product:

$$
\mathrm{E}_{a}^{i k}=\underbrace{\mathrm{id} \otimes \cdots \otimes \mathrm{id}}_{a} \otimes \mathrm{E}^{i k} \otimes \underbrace{\mathrm{id} \otimes \cdots \otimes \mathrm{id}}_{2 N-a} .
$$

This notation allows one to write down embeddings of operators on $\mathbb{C}^{2} \otimes \mathbb{C}^{2}$ into operators on $\mathfrak{h}_{0} \otimes \mathfrak{h}_{\mathfrak{q}}$. For instance, $\mathrm{P}_{a b}$ stands for the embedding of the permutation operator $\mathrm{P}$ on $\mathbb{C}^{2} \otimes \mathbb{C}^{2}$ into operators on $\mathfrak{h}_{0} \otimes \mathfrak{h}_{\mathfrak{q}}: \mathrm{P}_{a b}$ acts as $\mathrm{P}$ on $\mathfrak{h}_{a} \otimes \mathfrak{h}_{b}$ and as the identity operator on the other spaces in the tensor product decomposition of $\mathfrak{h}_{0} \otimes \mathfrak{h}_{\mathfrak{q}}$. This operator, along with its partial transpose on the $a^{\text {th }}$ space $\mathrm{P}_{a b}^{\mathrm{t}_{a}}$, can be recast in terms of the elementary matrices introduced above as

$$
\mathrm{P}_{a b}=\sum_{i, k=1}^{2} \mathrm{E}_{a}^{i k} \mathrm{E}_{b}^{k i}, \quad \mathrm{P}_{a b}^{\mathrm{t}_{a}}=\sum_{i, k=1}^{2} \mathrm{E}_{a}^{i k} \mathrm{E}_{b}^{i k}
$$

The explicit expression for the six-vertex R-matrix (1.10) ensures that

$$
\mathrm{R}_{a b}\left(-\frac{\kappa}{N}\right)=\mathrm{P}_{a b}+\mathrm{N}_{a b},
$$

where

$$
\mathrm{N}_{a b}=\sum_{i, k=1}^{2} n_{i k} \cdot \mathrm{E}_{a}^{i i} \mathrm{E}_{b}^{k k} \quad \text { with }\left\{\begin{array}{l}
n_{11}=n_{22}=\cosh \left(\frac{\aleph}{N}\right)-1-\operatorname{coth}(\eta) \sinh \left(\frac{\aleph}{N}\right) \\
n_{12}=n_{21}=-\sinh \left(\frac{\aleph}{N}\right) / \sinh (\eta)
\end{array} .\right.
$$

Define the operators

$$
\Pi_{\ell}=\mathrm{P}_{2 \ell 0}^{\mathrm{t}_{2 \ell}} \mathrm{P}_{02 \ell-1}
$$

and

$$
\mathrm{W}_{\ell}=\mathrm{R}_{2 \ell 0}^{\mathrm{t}_{2 \ell}}\left(-\frac{\aleph}{N}\right) \cdot \mathrm{R}_{02 \ell-1}\left(-\frac{\aleph}{N}\right)-\Pi_{\ell} .
$$

Further, given $\ell \geq m$, define

$$
\Omega_{\ell ; m}=\left\{\begin{array}{ccc}
\Pi_{\ell} \cdots \Pi_{m+1} \cdot \mathrm{e}^{\frac{h}{2 T} \sigma_{0}^{z} \delta_{m 0}} & \text { if } \quad \ell \geq m+1 \\
\mathrm{e}^{\frac{h}{2 T} \sigma_{0}^{z} \delta_{m 0}} & \text { if } \quad \ell=m
\end{array},\right.
$$

where $\delta_{a b}$ stands for the Kronecker symbol.

Upon expressing each factor associated to a pair of spaces $\mathfrak{h}_{2 \ell-1} \otimes \mathfrak{h}_{2 \ell}$ in $(1.9)$ as $\mathfrak{W}_{\ell}+\Pi_{\ell}$ one obtains that

$$
\mathrm{T}_{\mathrm{q} ; 0}(0)=\Omega_{N ; 0}+\sum_{n=1}^{N} \sum_{\ell \in \mathcal{L}_{N}^{(n)}} \boldsymbol{O}_{\ell} \quad \text { with } \quad \boldsymbol{O}_{\ell}=\Omega_{N ; \ell_{n}} \cdot \mathrm{W}_{\ell_{n}} \cdot \Omega_{\ell_{n}-1 ; \ell_{n-1}} \cdots \mathrm{W}_{\ell_{1}} \cdot \Omega_{\ell_{1}-1 ; 0},
$$

$\mathrm{T}_{\mathrm{q} ; 0}(\xi)$ as given in (1.9) and where the summation runs through

$$
\mathcal{L}_{N}^{(n)}=\left\{\boldsymbol{\ell}=\left(\ell_{1}, \ldots, \ell_{n}\right): 1 \leq \ell_{1}<\cdots<\ell_{n} \leq N\right\} .
$$


The decomposition (2.8) entails an analogous expansion for the quantum transfer matrix

$$
\mathrm{t}_{\mathrm{q}}=\omega_{N ; 0}+\delta \mathrm{t}_{\mathrm{q}},
$$

where

$$
\omega_{N ; 0}=\operatorname{Tr}_{0}\left[\Omega_{N ; 0}\right]
$$

and

$$
\delta \mathrm{t}_{\mathrm{q}}=\sum_{n=1}^{N} \sum_{\boldsymbol{\ell} \in \mathcal{L}_{N}^{(n)}} 0_{\ell} \quad \text { with } \quad 0_{\boldsymbol{\ell}}=\operatorname{tr}_{0}\left[\boldsymbol{O}_{\boldsymbol{\ell}}\right]
$$

It appears useful for further purposes to introduce a convention for writing vectors in $\mathfrak{h}_{\mathfrak{q}}=\bigotimes_{a=1}^{2 N} \mathfrak{h}_{a}$. Given vectors $\boldsymbol{v}_{1}, \ldots, \boldsymbol{v}_{2 N}$ in $\mathfrak{h}_{1}, \ldots, \mathfrak{h}_{2 N}$, we shall write

$$
\prod_{a=1}^{2 N} \boldsymbol{v}_{a}^{(a)}=\boldsymbol{v}_{1} \otimes \cdots \otimes \boldsymbol{v}_{2 N}
$$

viz. $\boldsymbol{v}_{a}^{(a)}$ stands for the $a^{\text {th }}$ vector appearing in the full tensor product. Further, we shall denote by $\boldsymbol{e}_{a}, a=1,2$, the canonical basis of $\mathbb{C}^{2}$ :

$$
\boldsymbol{e}_{1}=\left(\begin{array}{l}
1 \\
0
\end{array}\right) \quad, \quad \boldsymbol{e}_{2}=\left(\begin{array}{l}
0 \\
1
\end{array}\right) .
$$

Lemma 2.1. The operator $\omega_{N ; 0}$ introduced in (2.11) has rank 1 and takes the form

$$
\omega_{N ; 0}=\boldsymbol{v} \cdot \boldsymbol{w}^{\mathrm{t}} \quad \text { with }\left\{\begin{array}{rl}
\boldsymbol{v} & =\sum_{i \in\{1,2\}^{N}} \mathrm{e}^{\frac{h}{2 T} \varepsilon_{i N}} \prod_{s=1}^{N}\left\{\boldsymbol{e}_{i_{s}}^{(2 s)} \boldsymbol{e}_{i_{s-1}}^{(2 s-1)}\right\} \\
\boldsymbol{w} & =\sum_{j \in\{1,2\}^{N}} \prod_{s=1}^{N}\left\{\boldsymbol{e}_{j_{s}}^{(2 s)} \boldsymbol{e}_{j_{s}}^{(2 s-1)}\right\}
\end{array},\right.
$$

where $\varepsilon_{i}=(-1)^{i-1}$ and where we made use of periodic boundary conditions for the indices of $i=\left(i_{1}, \ldots, i_{N}\right)$, viz. $i_{0} \equiv i_{N}$. Furthermore, it holds that

$$
(\boldsymbol{w}, \boldsymbol{v})=2 \cosh \left(\frac{h}{2 T}\right), \quad\|\boldsymbol{v}\|^{2}=2^{N} \cosh \left(\frac{h}{T}\right) \quad \text { and } \quad\|\boldsymbol{w}\|^{2}=2^{N} .
$$

\section{Proof -}

Upon inserting the expression for the permutation operators in terms of elementary matrices (2.2), one obtains that the operator $\Omega_{\ell ; m}$ introduced in (2.7) can be expressed as

$$
\Omega_{\ell ; m}=\sum_{\substack{i_{s}, k_{s} \in\{1,2\} \\ s=2 m+1, \ldots, 2 \ell}} \prod_{s=2 m+1}^{2 \ell}\left\{\mathrm{E}_{s}^{i_{s} k_{s}}\right\} \cdot \mathrm{E}_{0}^{i_{2 \ell} k_{2 \ell}} \mathrm{E}_{0}^{k_{2 \ell-1} i_{2 \ell-1}} \cdots \mathrm{E}_{0}^{k_{2 m+1} i_{2 m+1}} \cdot \mathrm{e}^{\frac{h}{2 T} \varepsilon_{2 m+1} \delta_{m 0}}
$$

The algebra of elementary matrices $\mathrm{E}^{a b} \mathrm{E}^{c d}=\delta_{b c} \mathrm{E}^{a d}$ then allows one to simplify the product of elementary matrices over the auxiliary space 0 as

$$
\mathrm{E}_{0}^{i_{2 \ell} k_{2 \ell}} \mathrm{E}_{0}^{k_{2 \ell-1} i_{2 \ell-1}} \cdots \mathrm{E}_{0}^{k_{2 m+1} i_{2 m+1}}=\mathrm{E}_{0}^{i_{2} i_{2 m+1}} \cdot \prod_{s=m+1}^{\ell}\left\{\delta_{k_{2 s} k_{2 s-1}}\right\} \cdot \prod_{s=m+2}^{\ell}\left\{\delta_{i_{2 s-1} i_{2 s-2}}\right\} .
$$


The Kronecker symbols allow one to get rid of the summation over the odd labeled variables $i_{2 s-1}, s=m+2, \ldots, \ell$, and $k_{2 s-1}, s=m+1, \ldots, \ell$. Then, relabeling in the sum the even labeled variables as

$$
\left\{k_{2 s}, i_{2 s}\right\} \hookrightarrow\left\{k_{s}^{\prime}, i_{s}^{\prime}\right\}, \quad s=m+1, \ldots, \ell \quad \text { and } \quad i_{2 m+1} \hookrightarrow i_{m}^{\prime},
$$

one obtains

$$
\Omega_{\ell ; m}=\sum_{\left\{i_{s}\right\}_{m}^{\ell}} \sum_{\left\{k_{s}\right\}_{m+1}^{\ell}} \prod_{s=m+1}^{\ell}\left\{\mathrm{E}_{2 s}^{i_{s} k_{s}} \mathrm{E}_{2 s-1}^{i_{s-1} k_{s}}\right\} \cdot \mathrm{E}_{0}^{i i_{m}} \cdot \mathrm{e}^{\frac{h}{2 T} \varepsilon_{i_{m}} \delta_{m 0}} .
$$

Above, it is undercurrent that each $i_{s}$ or $k_{s}$ runs through the set $\{1,2\}$. At this stage, one can readily take the trace over the auxiliary space, hence leading to

$$
\omega_{N ; 0}=\sum_{\left\{i_{s}, k_{s}\right\}_{1}^{N}} \mathrm{e}^{\frac{h}{2 T} \varepsilon_{i}} \cdot \prod_{s=1}^{N}\left\{\mathrm{E}_{2 s}^{i_{s} k_{s}} \mathrm{E}_{2 s-1}^{i_{s-1} k_{s}}\right\}
$$

Here, we stress that the dependence on the id operator on the auxiliary space $\mathfrak{h}_{0}$ has been projected out in the elementary matrices appearing above. Finally, observe that an elementary matrix on $\mathcal{M}_{2}(\mathbb{C})$ can be recast as $\mathrm{E}^{a b}=\boldsymbol{e}_{a} \cdot\left(\boldsymbol{e}_{b}\right)^{\mathrm{t}}$. This decomposition entails that

$$
\prod_{s=1}^{N}\left\{E_{2 s}^{i_{s} k_{s}} E_{2 s-1}^{i_{s-1} k_{s}}\right\}=\prod_{s=1}^{N}\left\{\boldsymbol{e}_{i_{s}}^{(2 s)} \boldsymbol{e}_{i_{s-1}}^{(2 s-1)}\right\} \cdot\left\{\prod_{s=1}^{N} \boldsymbol{e}_{k_{s}}^{(2 s)} \boldsymbol{e}_{k_{s}}^{(2 s-1)}\right\}^{t} .
$$

The above factorisation allows one for a separation of the sums over the $i_{a}$ 's and the $k_{a}$ 's and leads to the claimed form of $\omega_{N ; 0}$. Finally, the value of the scalar product of $\boldsymbol{v}, \boldsymbol{w}$ along with their norms follows from direct calculations.

Observe that one has the representation

$$
\mathrm{W}_{\ell}=\sum_{i, k=1}^{2} \mathrm{M}_{\ell}^{i k} \mathrm{E}_{0}^{i k}, \quad \text { where } \quad \mathrm{M}_{\ell}^{i k}=\underbrace{\mathrm{id} \otimes \cdots \otimes \mathrm{id}}_{2 \ell-2} \otimes \mathrm{M}^{i k} \otimes \underbrace{\mathrm{id} \otimes \cdots \otimes \mathrm{id}}_{2 N-2 \ell}
$$

and

$$
\mathbb{M}^{i k}=\sum_{\alpha=1}^{4} \boldsymbol{v}_{i k ; \alpha} \cdot\left(\boldsymbol{w}_{i k ; \alpha}\right)^{\mathrm{t}}
$$

for some explicitly computable vectors $\boldsymbol{v}_{i k ; \alpha}, \boldsymbol{w}_{i k ; \alpha}$ which are normalised such that

$$
\left\|\boldsymbol{v}_{i k ; \alpha}\right\|=\frac{1}{2} \quad \text { and } \quad\left\|\boldsymbol{w}_{i k ; \alpha}\right\| \leq C_{\boldsymbol{w}}\left|\frac{\boldsymbol{\aleph}}{N}\right|
$$

for a constant $C_{w}>0$ and any $\alpha \in \llbracket 1 ; 4 \rrbracket$.

Lemma 2.2. Let $\boldsymbol{\ell}=\left(\ell_{1}, \ldots, \ell_{n}\right)$. Then the operator $O_{\ell}$ defined in (2.12) can be decomposed as

$$
O_{\ell}=\sum_{i, \widehat{j}_{\ell}} \sum_{\alpha_{\ell}} x_{i}^{\left(\alpha_{\ell}, \ell\right)} \cdot\left(y_{i, \hat{j}_{\ell}}^{\left(\alpha_{\ell}, \ell\right)}\right)^{t}
$$


Above, one sums over vectors

$$
\alpha_{\ell}=\left(\alpha_{\ell_{1}}, \ldots, \alpha_{\ell_{n}}\right) \in \llbracket 1 ; 4 \rrbracket^{n},
$$

and

$$
\boldsymbol{i}=\left(i_{1}, \ldots, i_{N}\right) \in\{1,2\}^{N} \quad, \quad \widehat{\boldsymbol{j}}_{\ell_{n}}=\left(j_{1}, \ldots, j_{\ell_{1}-1}, \widehat{j_{\ell_{1}}}, j_{\ell_{1}+1}, \ldots, \widehat{j_{\ell_{n}}}, \ldots, j_{N}\right) \in\{1,2\}^{N-n}
$$

The indicates the coordinates which are omitted. Furthermore, one has

$$
\begin{aligned}
& \boldsymbol{x}_{\boldsymbol{i}}^{\left(\boldsymbol{\alpha}_{\ell}, \ell\right)}=\mathrm{e}^{\frac{h}{2 T} \varepsilon_{i_{N}}} \prod_{\substack{s=1 \\
\neq \ell_{1}, \ldots, \ell_{n}}}^{N}\left\{\boldsymbol{e}_{i_{s}}^{(2 s)} \boldsymbol{e}_{i_{s-1}}^{(2 s-1)}\right\} \cdot \prod_{r=1}^{n} \boldsymbol{v}_{i_{\ell_{r}} i_{\ell_{r}-1} ; \alpha_{\ell_{r}}}^{\left(\ell_{r}\right)} \\
& \boldsymbol{y}_{\boldsymbol{i}, \hat{\boldsymbol{j}}_{\ell}}^{\left(\boldsymbol{\alpha}_{\ell}, \ell\right)}=\prod_{\substack{s=1 \\
\neq \ell_{1}, \ldots, \ell_{n}}}^{N}\left\{\boldsymbol{e}_{j_{s}}^{(2 s)} \boldsymbol{e}_{j_{s}}^{(2 s-1)}\right\} \cdot \prod_{r=1}^{n} \boldsymbol{w}_{i_{\ell_{r}} i_{\ell_{r}-1} ; \alpha_{\ell_{r}}}^{\left(\ell_{r}\right)}
\end{aligned}
$$

Note that, in the above expression for $\boldsymbol{x}_{\boldsymbol{i}}^{\left(\boldsymbol{\alpha}_{\ell}, \ell\right)}$, we use periodic boundary conditions for the indices of $\boldsymbol{i}$, viz. $i_{N} \equiv i_{0}$.

In (2.29), (2.30) $v_{i j ; \alpha}^{(\ell)}$, resp. $\boldsymbol{w}_{i j ; \alpha}^{(\ell)}$, means that the vector $\boldsymbol{v}_{i j ; \alpha}$, resp. $\boldsymbol{w}_{i j ; \alpha}$, appears on the position reserved to the spaces $\mathfrak{h}_{2 \ell-1} \otimes \mathfrak{h}_{2 \ell}$ in the tensor product decomposition of the full vector.

Proof - Inserting the expressions for each operator and using the representation (2.20) for $\Omega_{\ell ; m}$ yields that

$$
\begin{aligned}
& \boldsymbol{O}_{\boldsymbol{\ell}}=\sum_{\left\{\left\{i_{s}^{(r)}\right\}_{s=\ell_{r}}^{\ell_{r+1}-1}\right\}_{r=0}^{n}} \sum_{\left\{\left\{j_{s}^{(r)}\right\}_{s=\ell_{r+1}}^{\ell_{r+1}-1}\right\}_{r=0}^{n}} \sum_{\left\{q_{t}, p_{t}\right\}_{1}^{n}} \prod_{r=0}^{n} \prod_{s=\ell_{r}+1}^{\ell_{r+1}-1}\left\{\mathrm{E}_{2 s}^{i_{s}^{(r)} j_{s}^{(r)}} \mathrm{E}_{2 s-1}^{i_{s-1}^{(r)} j_{s}^{(r)}}\right\} \cdot \prod_{t=1}^{n} \mathrm{M}_{\ell_{t}}^{q_{t} p_{t}} \\
& \times \mathrm{E}_{0}^{i_{N}^{(n)} i_{\ell_{n}}^{(n)}} \mathrm{E}_{0}^{q_{n} p_{n}} \mathrm{E}_{0}^{i_{\ell_{n-1}}^{(n-1)} i_{\ell_{n-1}}^{(n-1)}} \mathrm{E}_{0}^{q_{n-1} p_{n-1}} \cdots \mathrm{E}_{0}^{q_{1} p_{1}} \mathrm{E}_{0}^{i_{\ell_{1}-1}^{(0)} i_{0}^{(0)}} \mathrm{e}^{\frac{h}{2 T} \varepsilon_{i_{0}}^{(0)}} .
\end{aligned}
$$

Here, we agree upon $\ell_{0}=0$ and $\ell_{n+1}=N+1$. The last line can be simplified as

$$
\mathrm{E}_{0}^{i_{N}^{(n)} i_{\ell_{n}}^{(n)}} \mathrm{E}_{0}^{q_{n} p_{n}} \mathrm{E}_{0}^{i_{\ell_{n-1}}^{(n-1)} i_{\ell_{n-1}^{(n-1)}}^{(n-1}} \mathrm{E}_{0}^{q_{n-1} p_{n-1}} \cdots \mathrm{E}_{0}^{q_{1} p_{1}} \mathrm{E}_{0}^{i_{\ell_{1}-1}^{(0)} i_{0}^{(0)}}=\mathrm{E}_{0}^{i_{N}^{(n)} i_{0}^{(0)}} \cdot \prod_{s=1}^{n}\left\{\delta_{\ell_{\ell_{s}}^{(s)}} q_{s}\right\} \cdot \prod_{s=1}^{n}\left\{\delta_{p_{s} i_{\ell_{s}-1}^{(s-1)}}\right\} .
$$

These Kronecker symbols allow one to compute the sums over the $p_{t}$ and $q_{t}$ indices. In order to have a more compact expression it is useful to change the summation variables as

$$
\begin{array}{ccccc}
i_{a}=i_{a}^{(r)} & \text { if } & \ell_{r} \leq a \leq \ell_{r+1}-1 & \text { with } & a \in \llbracket 0 ; N \rrbracket \\
j_{a}=j_{a}^{(r)} & \text { if } & \ell_{r} \leq a \leq \ell_{r+1}-1 & \text { with } & a \in \llbracket 1 ; N \rrbracket \backslash\left\{\ell_{1}, \ldots, \ell_{n}\right\}
\end{array}
$$

Then, one has

$$
\boldsymbol{O}_{\boldsymbol{\ell}}=\sum_{\left\{i_{a}\right\}_{a=0}^{N}} \sum_{\substack{\left\{j_{a}\right\}_{a=1}^{N} \\ a \neq \ell_{1}, \ldots, \ell_{n}}} \prod_{\substack{s=1 \\ \neq \ell_{1}, \ldots, \ell_{n}}}^{N}\left\{\mathrm{E}_{2 s}^{i_{s} j_{s}} \mathrm{E}_{2 s-1}^{i_{s-1} j_{s}}\right\} \cdot \prod_{r=1}^{n}\left\{\mathrm{M}_{\ell_{r}}^{i_{\ell} i_{r}-1}\right\} \cdot \mathrm{E}_{0}^{i_{N} i_{0}} \mathrm{e}^{\frac{h}{2 T} \varepsilon_{i}}
$$


At this stage, one may readily take the trace over the auxiliary space $\mathfrak{h}_{0}$. Upon projecting out the dependence on the id operator on $\mathfrak{h}_{0}$, it remains to observe that

$$
\begin{aligned}
\prod_{\substack{s=1 \\
\neq \ell_{1}, \ldots, \ell_{n}}}^{N}\left\{\mathrm{E}_{2 s}^{i_{s} j_{s}} \mathrm{E}_{2 s-1}^{i_{s-1} j_{s}}\right\} \cdot \prod_{r=1}^{n}\left\{\mathrm{M}_{\ell_{r}}^{i_{\ell_{r}} i_{\ell_{r}-1}}\right\} \\
\quad=\sum_{\alpha_{\ell}} \prod_{\substack{s=1 \\
\neq \ell_{1}, \ldots, \ell_{n}}}^{N}\left\{\boldsymbol{e}_{i_{s}}^{(2 s)} \boldsymbol{e}_{i_{s-1}}^{(2 s-1)}\right\} \cdot \prod_{r=1}^{n} \boldsymbol{v}_{i_{\ell_{r}} i_{\ell_{r-1}} ; \alpha_{\ell_{r}}}^{\left(\ell_{r}\right)} \cdot\left\{\prod_{\substack{s=1 \\
\neq \ell_{1}, \ldots, \ell_{n}}}^{N}\left\{\boldsymbol{e}_{j_{s}}^{(2 s)} \boldsymbol{e}_{j_{s}}^{(2 s-1)}\right\} \cdot \prod_{r=1}^{n} \boldsymbol{w}_{i_{\ell_{r}} i_{\ell_{r}-1} ; \alpha_{\ell_{r}}}^{\left(\ell_{r}\right)}\right\}^{\mathrm{t}}
\end{aligned}
$$

where $\alpha_{\ell}$ is as introduced in (2.27). This entails the claim.

Given two vectors $\ell \in \mathbb{N}^{n}, \boldsymbol{r} \in \mathbb{N}^{m}$, introduce the sets

$$
\mathcal{S}_{\ell}=\left\{\ell_{1}, \ldots, \ell_{n}\right\} \quad, \quad \mathcal{S}_{\boldsymbol{r}}=\left\{r_{1}, \ldots, r_{m}\right\} .
$$

Further define

$$
\begin{array}{ll}
\mathcal{S}_{\ell \cap r}=\mathcal{S}_{\ell} \cap \mathcal{S}_{r} & , \quad \mathcal{S}_{\ell \cup r}=\mathcal{S}_{\ell} \cup \mathcal{S}_{r} \\
\mathcal{S}_{\ell}^{\mathrm{c}}=\mathcal{S}_{\ell} \backslash \mathcal{S}_{\ell \cap r} & , \quad
\end{array}
$$

Lemma 2.3. Let

$$
\mathcal{S}_{\ell \cup r}=\left\{t_{1}, \ldots, t_{u}\right\} \quad \text { with } \quad 1 \leq t_{1}<\cdots<t_{u} \leq N .
$$

Then

$$
\begin{array}{r}
O_{\boldsymbol{\ell}} \cdot O_{\boldsymbol{r}}=\sum_{\boldsymbol{i}, \widehat{\boldsymbol{q}}_{\boldsymbol{r}}} \sum_{\boldsymbol{\alpha}_{\ell}, \boldsymbol{\beta}_{\boldsymbol{r}}}\left(2 \cosh \left[\frac{h}{2 T}\right]\right)^{\delta_{n, 0} \delta_{m, 0}} \cdot \boldsymbol{x}_{\boldsymbol{i}}^{\left(\boldsymbol{\alpha}_{\ell}, \boldsymbol{\ell}\right)} \cdot\left\{\sum_{\left\{p_{t_{a}}\right\}_{a=1}^{u}} \mathrm{e}^{\frac{h}{2 T} \varepsilon_{p_{t_{u}}}} \prod_{t_{k} \in \mathcal{S}_{\ell \cap r}}\left(\boldsymbol{w}_{i_{t_{k}} i_{t_{k}-1} ; \alpha_{t_{k}}}, \boldsymbol{v}_{p_{t_{k}} p_{t_{k-1}} ; \beta_{t_{k}}}\right)\right. \\
\left.\times \prod_{t_{k} \in \mathcal{S}_{\ell}^{\mathrm{c}}}\left(\boldsymbol{w}_{i_{t_{k}} i_{t_{k}-1} ; \alpha_{t_{k}}}, \boldsymbol{e}_{p_{t_{k-1}}} \otimes \boldsymbol{e}_{p_{t_{k}}}\right) \cdot \prod_{t_{k} \in \mathcal{S}_{\boldsymbol{r}}^{\mathrm{c}}}\left(\boldsymbol{u}, \boldsymbol{v}_{p_{t_{k}} p_{t_{k-1}} ; \beta_{t_{k}}}\right)\right\} \cdot\left(\boldsymbol{y}_{\boldsymbol{p}_{t}, \widehat{\boldsymbol{q}}_{\boldsymbol{r}}}^{\left(\boldsymbol{\beta}_{\boldsymbol{r}}, \boldsymbol{r}\right)}\right)^{\mathrm{t}} .
\end{array}
$$

Above, we use periodic boundary conditions on the indices of $t_{a}$, viz. $t_{0}=t_{u}$, we agree upon

$$
\boldsymbol{u}=\boldsymbol{e}_{1} \otimes \boldsymbol{e}_{1}+\boldsymbol{e}_{2} \otimes \boldsymbol{e}_{2},
$$

and have set

$$
\boldsymbol{p}_{t}=(\underbrace{p_{t_{u}}, \ldots, p_{t_{u}}}_{t_{1}-1}, \underbrace{p_{t_{1}}, \ldots, p_{t_{1}}}_{t_{2}-t_{1}}, \ldots, \underbrace{p_{t_{u-1}}, \ldots, p_{t_{u-1}}}_{t_{u}-t_{u-1}}, \underbrace{p_{t_{u}}, \ldots, p_{t_{u}}}_{N-t_{u}+1}) .
$$

Proof-

Obviously, one has

$$
0_{\ell} \cdot 0_{r}=\sum_{i, \widehat{j}_{\ell}} \sum_{\alpha_{\ell}} \sum_{p, \widehat{q}_{r}} \sum_{\beta_{r}} x_{i}^{\left(\alpha_{\ell}, \ell\right)} \cdot\left(y_{i, \widehat{j}_{\ell}}^{\left(\alpha_{\ell}, \ell\right)}, x_{p}^{\left(\beta_{r}, r\right)}\right) \cdot\left(y_{p, \widehat{q}_{r}}^{\left(\beta_{r}, r\right)}\right)^{t} .
$$


It further holds that

$$
\begin{aligned}
\left(\boldsymbol{y}_{\boldsymbol{i}, \widehat{\boldsymbol{j}}_{\boldsymbol{\ell}}}^{\left(\boldsymbol{\alpha}_{\boldsymbol{\ell}}, \boldsymbol{\ell}\right)}, \boldsymbol{x}_{\boldsymbol{p}}^{\left(\boldsymbol{\beta}_{\boldsymbol{r}}, \boldsymbol{r}\right)}\right)=\mathrm{e}^{\frac{h}{2 T} \varepsilon_{p_{N}}} \prod_{\substack{s=1 \\
\notin \mathcal{S}}}^{N}\left\{\delta_{j_{s} p_{s-1}} \delta_{p_{s} p_{s-1}}\right\} & \prod_{k \in \mathcal{S}_{\boldsymbol{\ell} \cap r}}\left(\boldsymbol{w}_{i_{k} i_{k-1} ; \alpha_{k}}, \boldsymbol{v}_{p_{k} p_{k-1} ; \beta_{k}}\right) \\
\times & \prod_{k \in \mathcal{S}_{\boldsymbol{\ell}}^{\mathrm{c}}}\left(\boldsymbol{w}_{i_{k} i_{k-1} ; \alpha_{k}}, \boldsymbol{e}_{p_{k-1}} \otimes \boldsymbol{e}_{p_{k}}\right) \cdot \prod_{s \in \mathcal{S}_{\boldsymbol{r}}^{\mathrm{C}}}\left(\boldsymbol{e}_{j_{s}} \otimes \boldsymbol{e}_{j_{s}}, \boldsymbol{v}_{p_{s} p_{s-1} ; \beta_{s}}\right) .
\end{aligned}
$$

The product over Kronecker symbols allows one to compute most of the sums over the $p_{a} \mathrm{~s}$ and $j_{a} \mathrm{~s}$ occurring in (2.42). We first treat the case when $\mathcal{S}_{\ell \cup r} \neq \emptyset$. To start with, consider the product

$$
\prod_{\substack{s=1 \\ \notin \mathcal{S}_{\mathcal{S} \cup r}}}^{N} \delta_{p_{s} p_{s-1}}=\prod_{s=1}^{t_{1}-1} \delta_{p_{s} p_{s-1}} \cdot \prod_{v=2}^{u} \prod_{s=t_{v-1}+1}^{t_{v}-1} \delta_{p_{s} p_{s-1}} \cdot \prod_{s=t_{u}+1}^{N} \delta_{p_{s} p_{s-1}} .
$$

Thus, the above string of Kronecker deltas will set

$$
p_{s}=p_{t_{v}} \quad \text { for } \quad s \in \llbracket t_{v} ; t_{v+1}-1 \rrbracket \quad v=1, \ldots, u-1 \quad \text { and } \quad p_{s}=p_{t_{u}} \quad \text { for } \quad s \in \llbracket 1 ; t_{1}-1 \rrbracket \cup \llbracket t_{u} ; N \rrbracket .
$$

Note that the disjoint interval for the $p_{t_{u}}$ variable comes from the boundary conditions $p_{0}=p_{N}$ on the indices of $\boldsymbol{p}$. Thus, the summation over $\boldsymbol{p}$ reduces to one over $p_{t_{1}}, \ldots, p_{t_{u}}$.

Recall that the indices $\ell_{1}, \cdots, \ell_{n}$ are absent in $\widehat{\boldsymbol{j}}_{\ell}$. Thus,

$$
\prod_{\substack{s=1 \\ \notin \mathcal{S}}}^{N} \delta_{j_{s} p_{s}}
$$

leaves free only the variables $j_{a}$ with $a \in \mathcal{S}_{r}^{\mathrm{c}}$. These variables only appear in the last scalar product in (2.43). Hence, by linearity one can pull the summation over each such variable into the corresponding scalar product. This yields the vectors $\boldsymbol{u}$ appearing in the last line of (2.39).

Finally, when $\mathcal{S}_{\ell \cup r}=\emptyset$, the summation over $\widehat{\boldsymbol{j}}_{\ell}$ can be explicitly performed, while the summation over then $p_{a}$ s reduces to the summation over $p_{N}$. Then, due to the presence of the weight factor $\mathrm{e}^{\frac{h}{2 T} \varepsilon_{p_{N}}}$ in $(2.43)$, one obtains $2 \cosh [h / 2 T]$.

One can straightforwardly generalise the above result to the computation of any product of $0_{\ell}$ operators for any choice of vectors $\boldsymbol{\ell}$.

Corollary 2.1. Let $n_{1}, \ldots, n_{M} \in \mathbb{N}$ be given and consider, for every $n_{s}>0$, a vector $\boldsymbol{\ell}^{(s)}=\left(\ell_{1}^{(s)}, \ldots, \ell_{n_{s}}^{(s)}\right)$ with components $1 \leq \ell_{1}^{(s)}<\cdots<\ell_{n_{s}}^{(s)} \leq N$. Further, denote

$$
\mathcal{S}_{\boldsymbol{\ell}^{(k)} \cup \boldsymbol{\ell}^{(k+1)}}=\left\{t_{1 ; k+1}, \ldots, t_{u_{k+1} ; k+1}\right\} .
$$

Then, upon denoting $t_{0 ; k+1}=t_{u_{k+1} ; k+1}$, it holds that

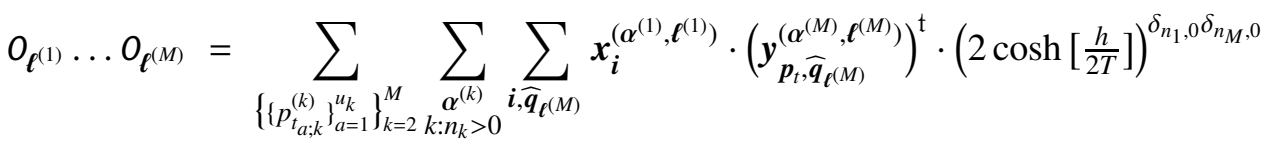

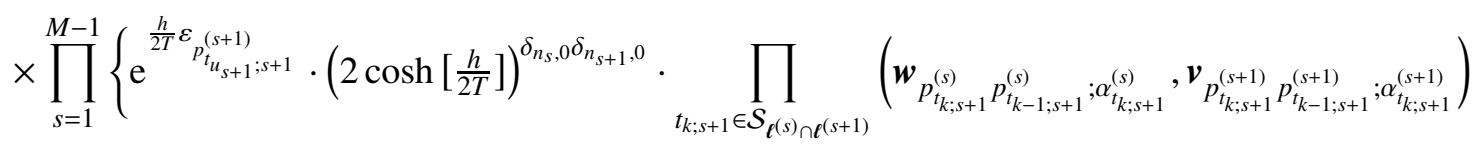

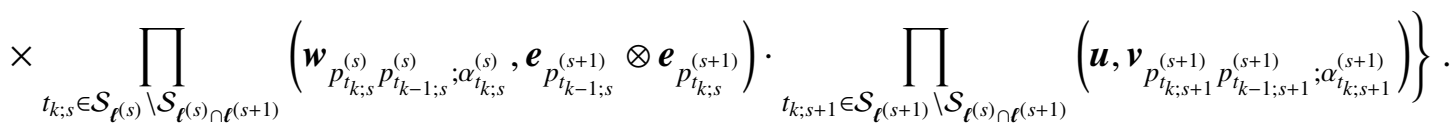


Here, one parameterises

$$
\boldsymbol{\alpha}^{(k)}=\left(\alpha_{t_{1 ; k}}^{(k)}, \ldots, \alpha_{t_{u_{k} ; k}}^{(k)}\right)
$$

and agrees upon the convention

$$
p_{t_{a ; k}}^{(1)}=i_{t_{a ; k}} \quad \text { and } \quad p_{t}=(\underbrace{p_{t_{u_{M}, M}}^{(M)}, \ldots, p_{t_{u_{M}, M}}^{(M)}}_{t_{1, M}-1}, \underbrace{p_{t_{1, M}}^{(M)}, \ldots, p_{t_{1, M}}^{(M)}}_{t_{2, M}-t_{1, M}}, \ldots, \underbrace{p_{t_{u_{M}-1, M}}^{(M)}, \ldots, p_{t_{u_{M}-1, M}}^{(M)}}_{t_{u_{M}, M}-t_{u_{M}-1, M}}, \underbrace{(M)}_{N-t_{u_{M}, M}+1}, \ldots, p_{t_{u_{M}, M}, M}^{(M)}) .
$$

Likewise, one has

$$
\begin{aligned}
& \operatorname{tr}_{\mathfrak{h}_{\mathfrak{q}}}\left[O_{\boldsymbol{\ell}^{(1)}} \ldots O_{\boldsymbol{\ell}^{(M)}}\right]=\sum_{\left\{\left\{p_{t_{a ; k}}^{(k)}\right\}_{a=1}^{u_{k}}\right\}_{k=1}^{M}} \sum_{\substack{\boldsymbol{\alpha}^{(k)} \\
k: n_{k}>0}} \prod_{s=1}^{M}\left(2 \cosh \left[\frac{h}{2 T}\right]\right)^{\delta_{n_{s}, 0} \delta_{n_{s+1}, 0}}
\end{aligned}
$$

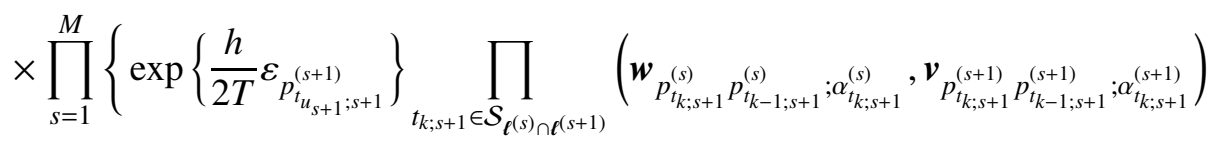

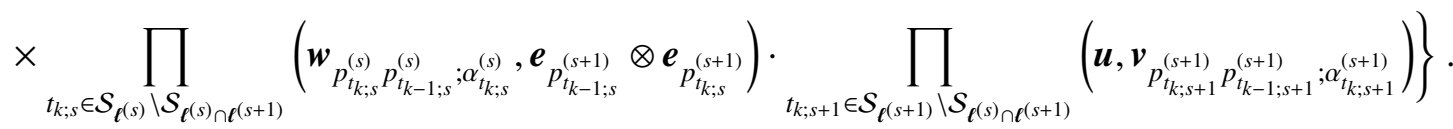

Proposition 2.2. There exists an $N$-independent constant $C>0$ such that the spectral radius of the operator $\delta t_{\mathrm{q}}$ appearing in the decomposition of the quantum transfer matrix (2.10) is bounded as

$$
r_{S}\left(\delta t_{\mathrm{q}}\right) \leq C \cdot|\boldsymbol{\aleph}| .
$$

Furthermore, there exist constants $C, C^{\prime}>0$ such that, for any $n \in \mathbb{N}$,

$$
\left|\operatorname{tr}_{\mathfrak{b}_{\mathfrak{q}}}\left[\omega_{N ; 0} \cdot\left(\delta t_{\mathfrak{q}}\right)^{n}\right]\right| \leq C^{\prime} \cdot(C \cdot|\mathbf{N}|)^{n},
$$

where $\omega_{N ; 0}$ has been introduced in 2.11).

More generally, there exist constants $C, C^{\prime}>0$ such that, for any $\ell_{1}, \ldots, \ell_{n} \in \mathbb{N}$,

$$
\left|\operatorname{tr}_{\mathfrak{h}_{\mathfrak{q}}}\left[\omega_{N ; 0} \cdot\left(\delta t_{\mathfrak{q}}\right)^{\ell_{1}} \omega_{N ; 0} \cdots \omega_{N ; 0} \cdot\left(\delta t_{\mathfrak{q}}\right)^{\ell_{n}}\right]\right| \leq\left(C^{\prime}\right)^{n} \cdot \prod_{a=1}^{n}(C \cdot|\boldsymbol{\aleph}|)^{\ell_{a}} .
$$

\section{Proof -}

It follows from the expansion (2.12) that

$$
\left(\delta \mathrm{t}_{\mathfrak{q}}\right)^{M}=\sum_{\substack{\left\{n_{s}\right\}_{s=1}^{M} \\ n_{s}=1}}^{N} \sum_{\substack{\left\{\ell^{(s)}\right\}_{s=1}^{M} \in \mathcal{\ell}_{N}^{(s)} \\ \boldsymbol{\ell}^{\left(n_{s}\right)}}} 0_{\boldsymbol{\ell}^{(1)}} \ldots 0_{\boldsymbol{\ell}^{(M)}}
$$

The expression obtained for the products of operators $0_{\boldsymbol{\ell}^{(1)}} \ldots 0_{\boldsymbol{\ell}^{(M)}}$ in Corollary 2.1, eqn. (2.48), leads to the bound

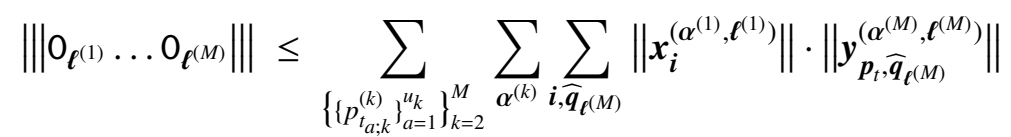

$$
\begin{aligned}
& \times \prod_{s=1}^{M-1}\left\{\exp \left\{\frac{h}{2 T} \varepsilon_{p_{t_{u s+1} ; s+1}^{(s+1)}}\right\} \cdot \prod_{t_{k ; s} \in \mathcal{S}_{\ell^{(s)}}}\left\|\boldsymbol{w}_{p_{t_{k ; s}}^{(s)}} p_{t_{k-1 ; s}}^{(s)} ; \alpha_{t_{k ;}}^{(s)}\right\| \cdot \prod_{t_{k ; s+1} \in \mathcal{S}_{\ell^{(s+1)}}}\left\{2\left\|\boldsymbol{v}_{p_{t_{k} ; s+1}^{(s+1)} p_{t_{k-1 ; s+1}}^{(s+1)} ; \alpha_{t_{k ; s+1}}^{(s+1)}}\right\|\right\}\right\} .
\end{aligned}
$$


Here we took into account that $n_{k}>0$ for any $k$ and $\|\boldsymbol{u}\|=2$. Thus, upon recalling the estimates (2.25) on $\boldsymbol{w}_{i j ; \alpha}$, $\boldsymbol{v}_{i j ; \alpha}$ and the expression for the vectors $\boldsymbol{x}_{i}^{(\alpha, \ell)}(2.29)$ and $\boldsymbol{y}_{\boldsymbol{p}, \overline{\boldsymbol{q}}_{\boldsymbol{r}_{m}}}^{(\alpha, \ell)}(2.30)$, one obtains

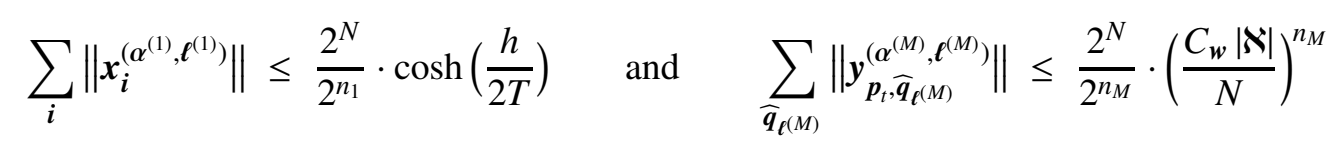

and thus

$$
\left\|\mid 0_{\boldsymbol{\ell}^{(1)}} \ldots 0_{\boldsymbol{\ell}^{(M)}}\right\| \leq \leq \prod_{k=1}^{M}\left\{4^{n_{k}}\right\} \cdot 2^{2 N-n_{M}-n_{1}} \cdot \cosh \left(\frac{h}{2 T}\right) \cdot\left(\frac{C_{\boldsymbol{w}}|\boldsymbol{\aleph}|}{N}\right)^{n_{M}} \cdot \prod_{s=1}^{M-1}\left\{2^{u_{s}} \cosh \left(\frac{h}{2 T}\right) \cdot\left(\frac{C_{\boldsymbol{w}}|\boldsymbol{\aleph}|}{N}\right)^{n_{s}}\right\} .
$$

Since $u_{k} \leq n_{k}+n_{k+1}$, this eventually leads to

$$
\left\|\left|0_{\boldsymbol{\ell}^{(1)}} \ldots 0_{\boldsymbol{\ell}^{(M)}}\right|\right\| \leq 2^{2 N} \cdot\left[\cosh \left(\frac{h}{2 T}\right)\right]^{M} \times \prod_{s=1}^{M}\left\{16 \cdot \frac{C_{\boldsymbol{w}}|\boldsymbol{\kappa}|}{N}\right\}^{n_{s}} .
$$

Inserting the latter bound into the series and using that

$$
\sum_{1 \leq \ell_{1}<\cdots<\ell_{n} \leq N} 1 \leq \frac{1}{n !} \sum_{\ell_{1}, \ldots, \ell_{n}=1}^{N} 1=\frac{N^{n}}{n !}
$$

implies the estimate

$$
\left\|\left|\left(\delta \mathrm{t}_{\mathfrak{q}}\right)^{M} \|\right| \leq 2^{2 N} \cdot\left[\cosh \left(\frac{h}{2 T}\right)\right]^{M} \sum_{\substack{\left\{n_{s}\right\}_{s=1}^{M} \\ n_{s}=1}}^{N} \prod_{s=1}^{M} \frac{\left\{16 \cdot C_{\boldsymbol{w}}|\boldsymbol{\aleph}|\right\}^{n_{s}}}{n_{s} !} \leq 2^{2 N}\left\{\cosh \left(\frac{h}{2 T}\right)\left(\mathrm{e}^{|\boldsymbol{N}| \widetilde{C}}-1\right)\right\}^{M},\right.
$$

for some $N$-independent $\widetilde{C}>0$. Hence

$$
r_{S}\left(\delta \mathrm{t}_{\mathrm{q}}\right)=\limsup _{M \rightarrow+\infty}\left\{\|\left.\left|\left(\delta \mathrm{t}_{\mathrm{q}}\right)^{M}\right|\right|^{\frac{1}{M}}\right\} \leq \cosh \left(\frac{h}{2 T}\right)\left(\mathrm{e}^{|\boldsymbol{N}| \widetilde{C}}-1\right) .
$$

This readily entails the bound (2.52).

The second bound (2.53) is obtained in an analogous way upon using the representation (2.51) and specialising the dimension $n_{1}$ associated with the vector $\boldsymbol{\ell}^{(1)}$ to zero. The same strategy yields (2.54) as well.

\section{Existence of a dominant Eigenvalue and commutativity of limits}

\subsection{Bound on the dominant and subdominant Eigenvalues}

Since the quantum transfer matrix $t_{\mathfrak{q}}$ has real-valued entries, its characteristic polynomial has real coefficients which entails that the Eigenvalues of the quantum transfer matrix $t_{q}$ are either real or appear in complex conjugate pairs.

Proposition 3.1. The largest modulus Eigenvalue $\widehat{\Lambda}_{\max }$ of the quantum transfer matrix $t_{\mathrm{q}}$ is non-degenerate, real and satisfies the estimate

$$
\widehat{\Lambda}_{\max }=2+\mathrm{O}\left(T^{-1}\right) \text {, }
$$

this uniformly in $N$. All the other Eigenvalues $\widehat{\Lambda}_{a}, a=1, \ldots, 2^{2 N}-1$ repeated according to their multiplicities, satisfy

$$
\widehat{\Lambda}_{a}=\mathrm{O}\left(T^{-1}\right) \quad \text { uniformly in } N .
$$




\section{Proof-}

Proposition 2.2 ensures that the operator $\delta \mathrm{t}_{\mathrm{q}}$ appearing in $\mathrm{t}_{\mathrm{q}}=\omega_{N ; 0}+\delta \mathrm{t}_{\mathrm{q}}$ has its spectral radius such that

$$
r_{S}\left(\delta \mathrm{t}_{\mathrm{q}}\right)=\mathrm{O}\left(T^{-1}\right), \quad \text { uniformly in } N \text {. }
$$

Then, $\lambda-\delta \mathrm{t}_{\mathrm{q}}$ is invertible for any $\lambda \notin \sigma\left(\delta \mathrm{t}_{\mathrm{q}}\right)$, the spectrum of $\delta \mathrm{t}_{\mathrm{q}}$. Thus, for such $\lambda \mathrm{s}$, it holds that

$$
\operatorname{det}\left[\lambda-\mathrm{t}_{\mathrm{q}}\right]=\operatorname{det}\left[\lambda-\delta \mathrm{t}_{\mathrm{q}}\right] \cdot \operatorname{det}\left[\operatorname{id}-\left(\lambda-\delta \mathrm{t}_{\mathrm{q}}\right)^{-1} \boldsymbol{\omega}_{N ; 0}\right]=\operatorname{det}\left[\lambda-\delta \mathrm{t}_{\mathrm{q}}\right] \cdot\left\{1-\left(\boldsymbol{w},\left[\lambda-\delta \mathrm{t}_{\mathrm{q}}\right]^{-1} \boldsymbol{v}\right)\right\},
$$

where we used the explicit expression for $\omega_{N ; 0}$ given in Lemma 2.1. The estimates obtained in Proposition 2.2 , (2.53), can be recast as

$$
\left|\left(w,\left(\delta \mathrm{t}_{\mathrm{q}}\right)^{n} v\right)\right| \leq\left(\frac{C}{T}\right)^{n}
$$

for some $N$-independent constant $C>0$. Thus, the series

$$
\mathcal{S}(\lambda)=\frac{1}{\lambda} \sum_{n \geq 0}\left(\boldsymbol{w},\left(\lambda^{-1} \delta \mathrm{t}_{\mathfrak{q}}\right)^{n} \boldsymbol{v}\right)
$$

converges uniformly on the set

$$
\left\{\lambda \in \mathbb{C}:|\lambda|>C(1+\epsilon) T^{-1}\right\}, \quad \text { this for any } \epsilon>0 .
$$

Furthermore, since the series

$$
\frac{1}{\lambda} \sum_{n \geq 0}\left(\frac{\delta \mathrm{t}_{\mathfrak{q}}}{\lambda}\right)^{n}
$$

converges in the operator norm to $\left[\lambda-\delta \mathrm{t}_{\mathrm{q}}\right]^{-1}$ on the set

$$
\left\{\lambda \in \mathbb{C}:|\lambda|>|| \delta \mathrm{t}_{\mathrm{q}} \| \mid\right\}, \quad \text { one has that } \mathcal{S}(\lambda)=\left(\boldsymbol{w},\left[\lambda-\delta \mathrm{t}_{\mathrm{q}}\right]^{-1} \boldsymbol{v}\right)
$$

on this set. Thus, since $\lambda \mapsto\left[\lambda-\delta \mathrm{t}_{\mathrm{q}}\right]^{-1}$ is analytic on $\mathbb{C} \backslash \sigma\left(\delta \mathrm{t}_{\mathrm{q}}\right)$, by uniqueness of the analytic continuation, it holds that

$$
\mathcal{S}(\lambda)=\left(\boldsymbol{w},\left[\lambda-\delta \mathrm{t}_{\mathrm{q}}\right]^{-1} \boldsymbol{v}\right) \quad \text { for any }|\lambda|>C \cdot(1+\epsilon) \cdot T^{-1} .
$$

The bound (3.5) also entails that

$$
\mathcal{S}(\lambda)=\frac{1}{\lambda} \cdot \overbrace{(\boldsymbol{w}, \boldsymbol{v})}^{=2 \cosh \left(\frac{h}{2 T}\right)}+\mathrm{O}\left(\frac{1}{T \lambda^{2}}\right)
$$

with a differentiable remainder.

Let $\mathcal{S}_{0}(\lambda)=2 / \lambda$. Then, using (3.11), it is easy to see that for some $C>0$ large enough

$$
\left|\mathcal{S}(\lambda)-\mathcal{S}_{0}(\lambda)\right|<\left|1-\mathcal{S}_{0}(\lambda)\right| \quad \text { for } \lambda \in \partial \mathcal{D}_{2, \frac{C}{T}} .
$$

Since $1-\mathcal{S}_{0}(\lambda)$ admits a unique zero in $\mathcal{D}_{2, \frac{C}{T}}$ at $\lambda=2$ and since $1-\mathcal{S}_{0}(\lambda)$ does not vanish on $\partial \mathcal{D}_{2, \frac{C}{T}}$, it follows by the Rouché theorem that $1-\mathcal{S}(\lambda)$ admits a unique zero in $\mathcal{D}_{2, \frac{C}{T}}$. An analogous reasoning based on (3.5) and applied to the domain $\mathbb{C} \backslash\left\{\mathcal{D}_{2, \frac{C}{T}} \cup \mathcal{D}_{0, \frac{C^{\prime}}{T}}\right\}$ with $C^{\prime}>0$ large enough implies that $1-\mathcal{S}$ has no zeroes in this domain. Thus, since the zeroes of $\lambda \mapsto \operatorname{det}\left[\lambda-\delta \mathrm{t}_{\mathrm{q}}\right]$ belong to the spectrum $\sigma\left(\delta \mathrm{t}_{\mathfrak{q}}\right)$ of the operator $\delta \mathrm{t}_{\mathfrak{q}}$ and since $\sigma\left(\delta \mathrm{t}_{\mathrm{q}}\right) \subset \mathcal{D}_{0, \frac{C^{\prime \prime}}{T}}$ for some $C^{\prime \prime}$, it follows that the characteristic polynomial $\operatorname{det}\left[\lambda-\mathrm{t}_{\mathrm{q}}\right]$ has a non-degenerate zero in the disk $\mathcal{D}_{2, \frac{C}{T}}$ and all its other zeroes are contained in the disk $\mathcal{D}_{0, \frac{\widetilde{C}}{T}}$ with constants $C, \widetilde{C}$ being $N$-independent. This entails the claim. 


\subsection{Commutativity of the limits}

In this subsection, we establish a theorem allowing one to exchange the Trotter and the thermodynamic limits when computing the free energy. One of the ingredients of the proof is the lemma below which was established by M. Suzuki in [33].

\section{Lemma 3.1. Suzuki [33]}

Let $a_{N, L}$ be a sequence in $\mathbb{C}$ such that

- for any $L \in \mathbb{N}, \lim _{N \rightarrow+\infty} a_{N, L}=\alpha_{L}$;

- $\lim _{L \rightarrow+\infty} \alpha_{L}=\alpha$;

- $\lim _{L \rightarrow+\infty} a_{N, L}=z_{N}$, with a convergence holding uniformly in $N$.

Then, $\lim _{N \rightarrow+\infty} z_{N}$ exists and equals $\alpha$.

Theorem 3.2. There exists $T_{0}>0$ such that, for any $T \geq T_{0}$,

$$
\lim _{L \rightarrow+\infty} \lim _{N \rightarrow+\infty} \frac{1}{L} \ln \operatorname{tr}_{\mathfrak{b}_{\mathfrak{q}}}\left[t_{\mathfrak{q}}^{L}\right]=\lim _{N \rightarrow+\infty} \lim _{L \rightarrow+\infty} \frac{1}{L} \ln \operatorname{tr}_{\mathfrak{b}_{\mathfrak{q}}}\left[t_{\mathfrak{q}}^{L}\right] .
$$

Proof-

The aim is to apply Lemma (3.1) to the sequence

$$
\tau_{N, L}=\frac{1}{L} \ln \operatorname{tr}_{\mathfrak{h}_{\mathfrak{q}}}\left[\mathrm{t}_{\mathfrak{q}}^{L}\right] \text {. }
$$

The equality

$$
\lim _{N \rightarrow+\infty} \tau_{N, L}=\frac{1}{L} \ln \operatorname{tr}_{\mathfrak{h}_{X X Z}}\left[\mathrm{e}^{-\frac{1}{T} \mathrm{H}}\right]
$$

holds from the very way the quantum transfer matrix is built. Indeed, one can show through elementary algebra based on the quantum inverse scattering method (see [18] for details) that

$$
\operatorname{tr}_{\mathfrak{h}_{\mathfrak{q}}}\left[\mathrm{t}_{\mathfrak{q}}^{L}\right]=\operatorname{tr}_{\mathfrak{h}_{X X Z}}\left[\mathrm{e}^{-\frac{1}{T} \mathrm{H}+\mathrm{A}_{N}}\right]
$$

where $\left\|\mid \mathrm{A}_{N}\right\|=\mathrm{O}\left(N^{-1}\right)$, but with an estimate that is non-uniform in the volume $L$.

As discussed in the introduction, the existence of the limit

$$
-T \lim _{L \rightarrow+\infty} \frac{1}{L} \ln \operatorname{tr}_{\mathfrak{h}_{X X Z}}\left[\mathrm{e}^{-\frac{1}{T} \mathrm{H}}\right]
$$

defining the per-site free energy $f$ follows from standard considerations in rigorous statistical mechanics, see [28].

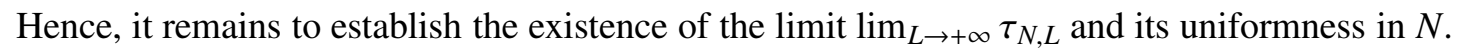

Following the notations and conclusions of Proposition 3.1, one has for $T$ large enough that

$$
\operatorname{tr}_{\mathrm{b}_{\mathfrak{q}}}\left[\mathrm{t}_{\mathrm{q}}^{L}\right]=\widehat{\Lambda}_{\max }^{L}+\sum_{a=1}^{22^{2 N}-1} \widehat{\Lambda}_{a}^{L} .
$$

Thus, it is clear that

$$
\lim _{L \rightarrow+\infty} \tau_{N, L}=\ln \left[\widehat{\Lambda}_{\max }\right] .
$$


In order to establish the uniformness in $N$ of this convergence, one should provide sharp bounds on the sum over the sub-dominant Eigenvalues.

Let $\mathfrak{P}$ denote the projector on the subspace of $\mathfrak{h}_{\mathfrak{q}}$ given as the direct sum of the Eigenspaces of $t_{\mathfrak{q}}$ associated with the subdominant Eigenvalues. Then, it holds that

$$
\operatorname{tr}_{\mathfrak{h}_{\mathfrak{q}}}\left[\left(\mathfrak{P} \mathrm{t}_{\mathfrak{q}} \mathfrak{P}\right)^{L}\right]=\sum_{a=1}^{2 N-1} \widehat{\Lambda}_{a}^{L}
$$

By using that $\mathfrak{P}^{2}=\mathfrak{P}$ and the cyclicity of the trace, one obtains

$$
\operatorname{tr}_{\mathfrak{h}_{\mathfrak{q}}}\left[\left(\mathfrak{B t} \mathrm{t}_{\mathfrak{q}} \mathfrak{P}\right)^{L}\right]=\operatorname{tr}_{\mathfrak{h}_{\mathfrak{q}}}\left[\left(\mathfrak{B} \mathrm{t}_{\mathfrak{q}}\right)^{L}\right] .
$$

The projector $\mathfrak{P}$ can be computed in an explicit form. Namely, standard considerations of functional calculus on the spectrum of an operator and Proposition 3.1 ensure that, for $T$ large enough, it holds that

$$
\mathfrak{P}=\oint_{\partial \mathcal{D}_{0,1}} \frac{\mathrm{d} \lambda}{2 \mathrm{i} \pi} \frac{1}{\lambda-\mathrm{t}_{\mathfrak{q}}} .
$$

Recall that $\mathrm{t}_{\mathrm{q}}=\omega_{N ; 0}+\delta \mathrm{t}_{\mathrm{q}}$ with $r_{S}\left(\delta \mathrm{t}_{\mathrm{q}}\right)=\mathrm{O}\left(T^{-1}\right)$ as established in Proposition 2.2. The latter ensures that the operator $\lambda-\delta \mathrm{t}_{\mathrm{q}}$ is invertible for any $\lambda \in \partial \mathcal{D}_{0,1}$, hence leading to the representation

$$
\frac{1}{\lambda-\mathrm{t}_{\mathrm{q}}}=\frac{1}{\lambda-\delta \mathrm{t}_{\mathrm{q}}} \cdot \frac{1}{\mathrm{id}-\left[\lambda-\delta \mathrm{t}_{\mathrm{q}}\right]^{-1} \cdot \omega_{N ; 0}} .
$$

Furthermore,

$$
\left[\lambda-\delta \mathrm{t}_{\mathrm{q}}\right]^{-1} \cdot \boldsymbol{\omega}_{N ; 0}=\boldsymbol{u} \cdot \boldsymbol{w}^{\mathrm{t}}, \quad \text { with } \quad \boldsymbol{u}=\left[\lambda-\delta \mathrm{t}_{\mathrm{q}}\right]^{-1} \cdot \boldsymbol{v},
$$

and by virtue of (3.10)- (3.11), one has $(\boldsymbol{w}, \boldsymbol{u})=2 \cdot \lambda^{-1}+\mathrm{O}\left(T^{-1}\right)$ uniformly in $\lambda \in \partial \mathcal{D}_{0,1}$ and in $N$. Then, it is easy to check that

$$
\frac{1}{\mathrm{id}-\left[\lambda-\delta \mathrm{t}_{\mathrm{q}}\right]^{-1} \cdot \omega_{N ; 0}}=\mathrm{id}+\frac{1}{1-\mathcal{S}(\lambda)}\left[\lambda-\delta \mathrm{t}_{\mathrm{q}}\right]^{-1} \cdot \omega_{N ; 0}
$$

with $\mathcal{S}$ as defined in (3.10). This yields

$$
\mathfrak{P}=\oint_{\partial \mathcal{D}_{0,1}} \frac{\mathrm{d} \lambda}{2 \mathrm{i} \pi} \frac{1}{\lambda-\delta \mathrm{t}_{\mathrm{q}}} \cdot\left\{\mathrm{id}+\frac{1}{1-\mathcal{S}(\lambda)}\left[\lambda-\delta \mathrm{t}_{\mathrm{q}}\right]^{-1} \cdot \omega_{N ; 0}\right\} .
$$

It is established in the proof of Proposition 3.1 that $1-\mathcal{S}$ admits a unique zero in $\mathbb{C} \backslash \mathcal{D}_{0,1}$ which corresponds to $\widehat{\Lambda}_{\text {max }}$. Thence, by taking the integral (3.26) by the residues lying outside of $\mathcal{D}_{0,1}$, one obtains

$$
\mathfrak{P}=\mathrm{id}+\frac{\left[\widehat{\Lambda}_{\max }-\delta \mathrm{t}_{\mathrm{q}}\right]^{-2} \cdot \omega_{N ; 0}}{\mathcal{S}^{\prime}\left(\widehat{\Lambda}_{\max }\right)} .
$$

Finally, one has the decomposition

$$
\mathfrak{P} \mathrm{t}_{\mathfrak{q}}=\mathfrak{I}_{0}+\delta \mathfrak{I} \quad \text { with }\left\{\begin{array}{l}
\mathfrak{I}_{0}=\mathfrak{P} \cdot \omega_{N ; 0} \\
\delta \mathfrak{I}=\mathfrak{P} \cdot \delta \mathrm{t}_{\mathfrak{q}}
\end{array},\right.
$$


which entails the expansion

$$
\operatorname{tr}_{\mathfrak{h}_{\mathfrak{q}}}\left[\left(\mathfrak{P t} \mathrm{t}_{\mathfrak{q}} \mathfrak{P}\right)^{L}\right]=\sum_{n=0}^{L} \sum_{\ell \in \mathcal{L}_{L}^{(n)}} \operatorname{tr}_{\mathfrak{h}_{\mathfrak{q}}}\left[\mathfrak{I}_{0}^{\ell_{1}-1} \cdot \delta \mathfrak{I} \cdot \mathfrak{I}_{0}^{\ell_{2}-\ell_{1}-1} \cdot \delta \mathfrak{I} \cdots \mathfrak{I}_{0}^{\ell_{n}-\ell_{n-1}-1} \cdot \delta \mathfrak{I} \cdot \mathfrak{I}_{0}^{L-\ell_{n}}\right] .
$$

Here $\boldsymbol{\ell}=\left(\ell_{1}, \ldots, \ell_{n}\right)$ and $\mathcal{L}_{L}^{(n)}$ is as introduced in (2.9).

In order to provide estimates for the summand, it is convenient to first recast $\mathfrak{I}_{0}$. By using that $\mathcal{S}\left(\widehat{\Lambda}_{\max }\right)=1$, one obtains that

$$
-\mathcal{S}^{\prime}\left(\widehat{\Lambda}_{\max }\right)=\frac{1}{\widehat{\Lambda}_{\max }} \cdot\left(1+s_{T}\right) \quad \text { with } \quad s_{T}=\frac{1}{\widehat{\Lambda}_{\max }} \sum_{n \geq 1} n\left(\boldsymbol{w},\left[\widetilde{\delta \mathrm{t}_{\mathrm{q}}}\right]^{n} \boldsymbol{v}\right) \quad \text { and } \quad \widetilde{\delta \mathrm{t}_{\mathrm{q}}}=\frac{\delta \mathrm{t}_{\mathfrak{q}}}{\widehat{\Lambda}_{\max }}
$$

The estimates (2.53) and (3.1) entail that $s_{T}=\mathrm{O}\left(T^{-1}\right)$ uniformly in $N$. Furthermore, one has the rewriting

$$
2 \cosh \left(\frac{h}{2 T}\right) \cdot\left(1+s_{T}\right)^{-1}=\widehat{\Lambda}_{\max }\left(1+w_{T}\right)
$$

where $w_{T}=\mathrm{O}\left(T^{-1}\right)$ owing to $\underline{3.1}$. This yields

$$
\mathfrak{I}_{0}=\left\{\mathrm{id}-\left[\mathrm{id}-{\widetilde{\delta \mathrm{t}_{\mathrm{q}}}}^{-2}\left(1+w_{T}\right)\right\} \cdot \omega_{N ; 0}=-\left\{w_{T}+\widetilde{\delta \mathrm{t}_{\mathrm{q}}} \cdot \frac{2-\widetilde{\delta \mathrm{t}_{\mathrm{q}}}}{\left[\mathrm{id}-\widetilde{\delta \mathrm{t}_{\mathrm{q}}}\right]^{2}} \cdot\left(1+w_{T}\right)\right\} \cdot \omega_{N ; 0} \cdot\right.
$$

This representation for $\mathfrak{I}_{0}$ is already enough so as to bound the summands in (3.29). Since the operator products appear under the trace, as in the proof of Proposition 3.1, one justifies that it is licit to expand all expressions of the form [id $\left.-\widetilde{\delta \mathrm{t}_{\mathrm{q}}}\right]^{-k}$ for some $k \in \mathbb{N}$ into power series in $\widetilde{\delta \mathrm{t}}_{\mathrm{q}}$ and to commute the trace with the summation symbols. Upon expanding each such factor, writing up the issuing sums, and then, in the very end, applying the bounds based on (2.54) followed by various resummations of the resulting sums, one easily concludes that bounding the trace

$$
\operatorname{tr}_{\mathfrak{b}_{\mathfrak{q}}}\left[\mathfrak{I}_{0}^{\ell_{1}-1} \cdot \delta \mathfrak{I} \cdot \mathfrak{I}_{0}^{\ell_{2}-\ell_{1}-1} \cdot \delta \mathfrak{I} \cdots \mathfrak{I}_{0}^{\ell_{n}-\ell_{n-1}-1} \cdot \delta \mathfrak{I} \cdot \mathfrak{I}_{0}^{L-\ell_{n}}\right]
$$

amounts to dropping the trace symbol and replacing each appearance of the operator $\omega_{N ; 0}$ in the product contained under the trace or by a constant $C_{1}>0$, each appearance of $w_{T}$ or $s_{T}$ by $C_{2} / T$ with a constant $C_{2}>0$, and each appearance of $\widetilde{\delta \mathrm{t}_{\mathrm{q}}}$ or $\delta \mathrm{t}_{\mathrm{q}}$ by $C_{3} / T$ with a constant $C_{3}>0$, viz.

$$
\left|\operatorname{tr}_{\mathfrak{r}_{\mathfrak{a}}}\left[\mathfrak{I}_{0}^{\ell_{1}-1} \cdot \delta \mathfrak{I} \cdot \mathfrak{I}_{0}^{\ell_{2}-\ell_{1}-1} \cdot \delta \mathfrak{I} \cdots \mathfrak{I}_{0}^{\ell_{n}-\ell_{n-1}-1} \cdot \delta \mathfrak{I} \cdot \mathfrak{I}_{0}^{L-\ell_{n}}\right]\right| \leq \mathcal{Z}_{0}^{\ell_{1}-1} \cdot \delta \mathcal{Z} \cdot \mathcal{Z}_{0}^{\ell_{2}-\ell_{1}-1} \cdot \delta \mathcal{Z} \cdots \mathcal{Z}_{0}^{\ell_{n}-\ell_{n-1}-1} \cdot \delta \mathcal{Z} \cdot \mathcal{Z}_{0}^{L-\ell_{n}}
$$

where

$$
\mathcal{Z}_{0}=C_{1}\left\{\frac{C_{2}}{T}+\frac{C_{3}}{T} \frac{2+\frac{C_{3}}{T}}{\left[1-\frac{C_{3}}{T}\right]^{2}} \cdot\left(1+\frac{C_{2}}{T}\right)\right\} \quad \text { and } \quad \delta \mathcal{Z}=\left\{1+C_{1} \frac{\left|\widehat{\Lambda}_{\max }\right|^{-1}}{\left(1-\frac{C_{2}}{T}\right) \cdot\left[1-\frac{C_{3}}{T}\right]^{2}}\right\} \cdot \frac{C_{3}}{T} .
$$

This immediately yields, for some $C, C^{\prime}>0$, the estimate

$$
\left|\operatorname{tr}_{\mathfrak{h}_{\mathfrak{q}}}\left[\mathfrak{I}_{0}^{\ell_{1}-1} \cdot \delta \mathfrak{I} \cdot \mathfrak{I}_{0}^{\ell_{2}-\ell_{1}-1} \cdot \delta \mathfrak{I} \cdots \mathfrak{I}_{0}^{\ell_{n}-\ell_{n-1}-1} \cdot \delta \mathfrak{I} \cdot \mathfrak{I}_{0}^{L-\ell_{n}}\right]\right| \leq\left(C^{\prime}\right)^{L-n}\left(\frac{C}{T}\right)^{L} .
$$

Thus, one obtains

$$
\left|\operatorname{tr}_{\mathfrak{f}_{\mathfrak{a}}}\left[\left(\mathfrak{P} \mathrm{t}_{\mathfrak{q}} \mathfrak{P}\right)^{L}\right]\right| \leq \sum_{n=0}^{+\infty} \frac{L^{n}}{n !} \cdot\left[C^{\prime}\right]^{L-n} \cdot\left(\frac{C}{T}\right)^{L}=\left(\frac{C C^{\prime} \mathrm{e}^{\frac{1}{C^{\prime}}}}{T}\right)^{L} .
$$


This bound allows one to obtain the estimate

$$
\left|\tau_{N, L}-\widehat{\Lambda}_{\max }\right|=\frac{1}{L}\left|\ln \left[1+\left(\widehat{\Lambda}_{\max }\right)^{-L} \cdot \sum_{a=1}^{2 N}-1 \widehat{\Lambda}_{a}^{L}\right]\right| \leq \frac{1}{L}\left(\frac{C^{\prime \prime}}{T}\right)^{L}
$$

for some $N$-independent constant $C^{\prime \prime}>0$, hence ensuring a uniform in $N$ convergence in (3.19). One is then able to conclude by virtue of Lemma 3.1 .

\section{The non-linear integral equation based description of the spectrum}

\subsection{Preliminary discussion}

The main advantage of rewriting the partition function as the Trotter limit involving the quantum transfer matrix (1.8) is that one may construct the Eigenvectors of $t_{\mathrm{q}}$ through the Bethe Ansatz [2, 15]. We stress that it has not been established whether the Bethe Ansatz provides one with a complete set of Eigenstates or not, although one may explicitly check that it is so when $\Delta=0$. The question of the completeness of the Bethe Ansatz is however not so relevant in that the computation of $f$ only demands to be able to construct the largest Eigenvalue by means of the Bethe Ansatz. Further information, such as the finite temperature correlation lengths, can also be accessed at least for the sub-dominant Eigenvalues which can be constructed by the Bethe Ansatz.

Consider $M$ complex numbers $\lambda_{1}, \ldots, \lambda_{M} \in \mathbb{C}$, distinct or not. If some of these numbers coincide, the total amount of these that is equal to a given complex number $z$ is called their multiplicity and denoted $k_{z}$. One then defines the set

$$
\left\{\lambda_{a}\right\}_{1}^{M}=\left\{\left(\lambda, k_{\lambda}\right): \lambda \in\left\{\lambda_{1}, \ldots, \lambda_{M}\right\}\right\} .
$$

In the Bethe Ansatz approach, one looks for Eigenstates of $t_{q}$ in the form of combinatorial sums parametrised in terms of $M$ complex numbers $\lambda_{1}, \ldots, \lambda_{M}$,

$$
\boldsymbol{\Psi}\left(\left\{\lambda_{a}\right\}_{a=1}^{M}\right) .
$$

In order for $\Psi\left(\left\{\lambda_{a}\right\}_{a=1}^{M}\right)$ to give rise to an Eigenstate of the quantum transfer matrix $\mathbf{t}_{q}$, the roots $\lambda_{1}, \ldots, \lambda_{M}$ should be admissible, namely satisfy

- $\lambda_{a} \neq \lambda_{b} \pm \mathrm{i} \zeta \bmod \mathrm{i} \pi \mathbb{Z}$ for any $a, b$,

- $\lambda_{a} \notin\{ \pm \boldsymbol{\aleph} / N, \pm \boldsymbol{\kappa} / N \pm \mathrm{i} \zeta\}$ for any $a$,

and, for any $a=1, \ldots, M$, solve the set of Bethe Ansatz equations:

$\mathrm{e}^{-\frac{h}{T}}(-1)^{s} \frac{\partial^{p}}{\partial \xi^{p}}\left\{\prod_{k=1}^{M}\left\{\frac{\sinh \left(\mathrm{i} \zeta-\xi+\lambda_{k}\right)}{\sinh \left(\mathrm{i} \zeta+\xi-\lambda_{k}\right)}\right\} \cdot\left\{\frac{\sinh (\xi-\boldsymbol{\aleph} / N) \sinh (\mathrm{i} \zeta+\xi+\boldsymbol{\aleph} / N)}{\sinh (\xi+\boldsymbol{\aleph} / N) \sinh (\mathrm{i} \zeta-\xi+\boldsymbol{\aleph} / N)}\right\}^{N}\right\}_{\mid \xi=\lambda_{a}}=-\delta_{p, 0}, \quad p=0, \ldots, k_{\lambda_{a}}-1$

where $k_{\lambda_{a}}$ is the multiplicity of $\lambda_{a}$ and one adds the subsidiary condition that the derivative does not vanish for $p=k_{\lambda_{a}}$. Further, $\boldsymbol{\aleph}$ corresponds to a reparametrisation of the temperature:

$$
\aleph=-\mathrm{i} J \frac{\sin (\zeta)}{T}
$$


and $s=N-M$ is called the spin. Note that, if $\lambda_{1}, \ldots, \lambda_{M}$ are all pairwise distinct and admissible, the system of Bethe Ansatz equations reduces to the usually encountered form

$$
\mathrm{e}^{-\frac{h}{T}}(-1)^{s} \prod_{k=1}^{M}\left\{\frac{\sinh \left(\mathrm{i} \zeta-\lambda_{a}+\lambda_{k}\right)}{\sinh \left(\mathrm{i} \zeta+\lambda_{a}-\lambda_{k}\right)}\right\} \cdot\left\{\frac{\sinh \left(\lambda_{a}-\boldsymbol{\aleph} / N\right) \sinh \left(\mathrm{i} \zeta+\lambda_{a}+\boldsymbol{\aleph} / N\right)}{\sinh \left(\lambda_{a}+\boldsymbol{\aleph} / N\right) \sinh \left(\mathrm{i} \zeta-\lambda_{a}+\boldsymbol{\aleph} / N\right)}\right\}^{N}=-1, \quad a=1, \ldots, M .
$$

When all of the above conditions are fulfilled, the vector (4.2) is associated with the Eigenvalue

$$
\begin{aligned}
\tau\left(\xi \mid\left\{\lambda_{k}\right\}_{1}^{M}\right)=(-1)^{N} \mathrm{e}^{\frac{h}{2 T}} & \prod_{k=1}^{M}\left\{\frac{\sinh \left(\xi-\lambda_{k}+\mathrm{i} \zeta\right)}{\sinh \left(\xi-\lambda_{k}\right)}\right\} \cdot\left(\frac{\sinh (\xi+\boldsymbol{\aleph} / N) \sinh (\xi-\boldsymbol{\aleph} / N-\mathrm{i} \zeta)}{\sinh ^{2}(-\mathrm{i} \zeta)}\right)^{N} \\
& +(-1)^{N} \mathrm{e}^{-\frac{h}{2 T}} \prod_{k=1}^{M}\left\{\frac{\sinh \left(\xi-\lambda_{k}-\mathrm{i} \zeta\right)}{\sinh \left(\xi-\lambda_{k}\right)}\right\} \cdot\left(\frac{\sinh (\xi+\boldsymbol{\aleph} / N+\mathrm{i} \zeta) \sinh (\xi-\boldsymbol{\aleph} / N)}{\sinh ^{2}(-\mathrm{i} \zeta)}\right)^{N}
\end{aligned}
$$

of the trace of the quantum monodromy matrix $\operatorname{tr}_{\mathfrak{y}_{0}}\left[\mathrm{~T}_{\mathrm{q} ; 0}(\xi)\right]$. See, e.g. [21] , for more details.

The system of Bethe Ansatz equation is highly non-trivial to solve, with the exception of the case $\zeta=\pi / 2$, viz. $\Delta=0$, where the equations decouple and can thus be solved explicitly. Furthermore, it appears hopeless to take directly the infinite Trotter number limit on the level of the Bethe equations (4.3). An alternative has been proposed in the literature [5, 21] . The idea consists in putting the problem of finding solutions to (4.3) in correspondence with the one of solving certain non-linear integral equations. The non-linear integral equations appear easier to deal with, be it with respect to a numerical calculation of the spectrum or relatively to various formal manipulations thereof, for instance the calculation of the infinite Trotter number limit.

In the following, we will make use of the notations

$$
\mathcal{S}_{\alpha}=\{z \in \mathbb{C}:|\mathfrak{J}(z)|<\alpha\} \quad \text { and } \quad \zeta_{\mathrm{m}}=\min \{\zeta, \pi-\zeta\} .
$$

Also, in order to state the non-linear integral equation based characterisation, it is convenient to introduce

$$
\theta(\lambda)=\left\{\begin{array}{clc}
i \ln \left(\frac{\sinh (i \zeta+\lambda)}{\sinh (i \zeta-\lambda)}\right) & \text { for } & |\mathfrak{I}(\lambda)|<\zeta_{\mathrm{m}} \\
-\pi \operatorname{sgn}(\pi-2 \zeta)+\mathrm{i} \ln \left(\frac{\sinh (i \zeta+\lambda)}{\sinh (\lambda-\mathrm{i} \zeta)}\right) & \text { for } & \zeta_{\mathrm{m}}<|\mathfrak{J}(\lambda)|<\pi / 2
\end{array}\right.
$$

where "ln" corresponds to the principal branch of the logarithm. The above definition makes $\theta$ an i $\pi$-periodic holomorphic function on $\mathbb{C} \backslash\left\{\mathbb{R}^{+} \pm \mathrm{i} \zeta_{\mathrm{m}}+\mathrm{i} \pi \mathbb{Z}\right\}$ with cuts on $\mathbb{R}^{+} \pm \mathrm{i} \zeta_{\mathrm{m}}+\mathrm{i} \pi \mathbb{Z}$. In the following, $\theta_{+}(z)$ will stand for the + boundary value of $\theta$, viz. the limit $\theta_{+}(z)=\lim _{\epsilon \rightarrow 0^{+}} \theta(z+\mathrm{i} \epsilon)$. This regularisation is only needed if $z \in\left\{\mathbb{R}^{+} \pm \mathrm{i} \zeta_{\mathrm{m}}+\mathrm{i} \pi \mathbb{Z}\right\}$.

Further, we introduce

$$
K(\xi)=\frac{1}{2 \pi} \theta^{\prime}(\xi)=\frac{\operatorname{sgn}(\pi-2 \zeta)}{2 \mathrm{i} \pi}\left\{\operatorname{coth}\left(\xi-\mathrm{i} \zeta_{\mathrm{m}}\right)-\operatorname{coth}\left(\xi+\mathrm{i} \zeta_{\mathrm{m}}\right)\right\}
$$

Finally, a set $\mathcal{M}$ will be called admissible if, for any $x, x^{\prime} \in \mathcal{M}$ it holds $x \neq x^{\prime} \pm \mathrm{i} \zeta \bmod \mathrm{i} \pi \mathbb{Z}$ and if $\pm \boldsymbol{\kappa} / N$ and $\pm \boldsymbol{\aleph} / N \pm \mathrm{i} \zeta$ do not belong to $\mathcal{M}, \bmod i \pi \mathbb{Z}$.

Proposition 4.1. Let the set $\left\{\lambda_{a}\right\}_{a=1}^{M}$ be built up form an admissible solution to the Bethe Ansatz equations (4.3). Then, there exists a bounded domain $\mathcal{D} \subset \mathcal{S}_{\zeta_{\mathrm{m}} / 2}$ containing $-\boldsymbol{\aleph} / N$, such that $\left\{\lambda_{a}\right\}_{a=1}^{M}$ allows one to construct a solution $(\widehat{\mathfrak{A}}, \widehat{\mathfrak{X}}, \widehat{\mathcal{Y}})$ to the below non-linear problem.

Find 
- $\widehat{\mathfrak{A}}$ piecewise continuous on $\partial \mathcal{D}$;

- a set $\widehat{\mathfrak{X}}=\left\{\widehat{x}_{a}\right\}_{1}^{|\widehat{\mathcal{X}}|}$ with $\widehat{x}_{a} \in \mathcal{D} \backslash\left\{\frac{\mathbb{N}}{N},-\frac{\aleph}{N}\right\}$, for $a=1, \ldots,|\widehat{\mathfrak{X}}|$;

- a set $\widehat{\mathcal{Y}}=\left\{\widehat{y}_{a}\right\}_{1}^{|\widehat{y}|}$ with $\widehat{y}_{a} \in \mathcal{S}_{\pi / 2} \backslash \overline{\mathcal{D}}$ for $a=1, \ldots,|\widehat{\mathcal{Y}}|$, and $\left\{\widehat{y}_{1}, \ldots, \widehat{y}_{|\widehat{y}|}\right\}$ being an admissible set;

such that

- $\mathrm{e}^{\widehat{A}}$ extends to a meromorphic, $\mathrm{i} \pi$-periodic function on $\mathbb{C}$ whose only poles in $\mathcal{D}$ build up exactly the set

$$
\widehat{y}_{\mathrm{sg}}=\left\{\left(y-\mathrm{i} \operatorname{sgn}(\pi-2 \zeta) \cdot \zeta_{\mathrm{m}}, k_{y}\right):\left(y, k_{y}\right) \in \widehat{y} \text { and } y-\mathrm{i} \operatorname{sgn}(\pi-2 \zeta) \cdot \zeta_{\mathrm{m}} \in \mathcal{D}\right\},
$$

the order of the pole at $y$ being given by the multiplicity $k_{y}$ of $\left(y, k_{y}\right) \in \widehat{y}$;

- $1+\mathrm{e}^{\widehat{\mathfrak{A}}}$ does not vanish on $\partial \mathcal{D}$;

- for any $\left(x, k_{x}\right) \in \widehat{\mathfrak{X}}$, resp. $\left(y, p_{y}\right) \in \widehat{\mathcal{Y}}$ :

$$
\begin{array}{llll}
\partial_{\xi}^{r}\left\{\mathrm{e}^{\widehat{\mathscr{A}}(\xi)}\right\}_{\mid \xi=x}=-\delta_{r, 0} & \text { for any } & x \in \widehat{\mathfrak{X}}, & r=0, \ldots, k_{x}-1 \\
\partial_{\xi}^{r}\left\{\mathrm{e}^{\widehat{\mathscr{A}}(\xi)}\right\}_{\mid \xi=y}=-\delta_{r, 0} & \text { for any } & x \in \widehat{\mathcal{Y}}, & r=0, \ldots, p_{y}-1
\end{array}
$$

and the derivatives do not vanish for $r=k_{x}$, resp. $r=p_{y}$;

- $\widehat{\mathfrak{A}}$ is subject to the monodromy constraint

$$
\oint_{\partial \mathcal{D}} \frac{\widehat{\mathfrak{A}}^{\prime}(u)}{1+\mathrm{e}^{-\widehat{\mathscr{A}}(u)}} \cdot \frac{\mathrm{d} u}{2 \mathrm{i} \pi}=-s-|\widehat{\mathcal{Y}}|-\left|\widehat{\mathcal{Y}}_{\mathrm{sg}}\right|+|\widehat{\mathfrak{X}}|
$$

for some $s \in \mathbb{Z}$;

- $\widehat{\mathfrak{A}}$ solves the non-linear integral equation

$$
\widehat{\mathfrak{A}}(\xi)=-\frac{h}{T}+\mathfrak{w}_{N}(\xi)-\mathrm{i} \pi s+\mathrm{i} \sum_{y \in \widehat{\mathbb{Y}}_{k}} \theta_{+}(\xi-y)+\oint_{\partial \mathcal{D}} K(\xi-u) \cdot \mathcal{L} \mathrm{n}\left[1+\mathrm{e}^{\widehat{\mathfrak{A}}}\right](u) \cdot \mathrm{d} u
$$

with $\xi \in \mathcal{S}_{\zeta_{\mathrm{m}} / 2}$ and where, for $v \in \partial \mathcal{D}$, one has

$$
\mathcal{L}\left[1+\mathrm{e}^{\widehat{\mathfrak{I}}}\right](v)=\int_{\kappa}^{v} \frac{\widehat{\mathfrak{A}}^{\prime}(u)}{1+\mathrm{e}^{-\widehat{\mathfrak{A}}(u)}} \cdot \mathrm{d} u+\ln \left[1+\mathrm{e}^{\widehat{\mathfrak{I}}(\kappa)}\right] .
$$

Here $\kappa$ is some point on $\partial \mathcal{D}$ and the integral is taken, in the positive direction along $\partial \mathcal{D}$, from $\kappa$ to $v$. The function " In" appearing above corresponds to the principal branch of the logarithm extended to $\mathbb{R}^{-}$with the convention $\arg (z) \in[-\pi ; \pi[$.

Finally, we have set

$$
\mathfrak{w}_{N}(\xi)=N \ln \left(\frac{\sinh (\xi-\boldsymbol{\aleph} / N) \sinh (\xi+\boldsymbol{\aleph} / N-\mathrm{i} \zeta)}{\sinh (\xi+\boldsymbol{\aleph} / N) \sinh (\xi-\boldsymbol{\aleph} / N-\mathrm{i} \zeta)}\right) .
$$


The sets appearing in the non-linear integral equation (4.13) are defined as

$$
\widehat{\mathbb{Y}}_{\kappa}=\widehat{\mathbb{Y}} \ominus\{\kappa\}^{\oplus(s+|\widehat{Y}|)} \quad \text { with } \quad \widehat{\mathbb{Y}}=\widehat{\mathcal{Y}} \oplus \widehat{\mathcal{Y}}_{\mathrm{sg}} \ominus \widehat{\mathfrak{X}},
$$

where we employed the conventions introduced in (1.24) and above (1.26). Finally, (4.13) also builds on the summation convention introduced in (1.25)-(1.26).

Conversely, any solution to the above non-linear problem gives rise to an admissible solution of the Bethe Ansatz equations (4.3).

The above proposition was first formulated and argued in [5] and subsequently developed in many works. Since the construction plays an important role in our analysis, we reproduce the proof for the reader's convenience.

Note also that $\mathcal{L} n\left[1+\mathrm{e}^{\widehat{\mathscr{A}}}\right]$ introduced above is well-defined since $1+\mathrm{e}^{\widehat{\mathscr{A}}}$ does not vanish on $\partial \mathcal{D}$. Furthermore, the definition of $\mathcal{L} \mathrm{n}$ implies that

$$
\mathrm{e}^{\mathcal{L}\left[1+\mathrm{e}^{\widehat{\mathbb{I}}}\right](v)}=1+\mathrm{e}^{\widehat{\mathfrak{A}}(v)}
$$

meaning that it provides one with a determination for the logarithm of $1+\mathrm{e}^{\widehat{\mathscr{A}}}$.

Proof-

Given a distribution of roots $\left\{\lambda_{a}\right\}_{1}^{M}$ solving the Bethe equations (4.3), one introduces an auxiliary function $\widehat{a}$ by the formula

$$
\widehat{\mathfrak{a}}(\xi)=\mathrm{e}^{-\frac{h}{T}}(-1)^{s} \prod_{k=1}^{M}\left\{\frac{\sinh \left(\mathrm{i} \zeta-\xi+\lambda_{k}\right)}{\sinh \left(\mathrm{i} \zeta+\xi-\lambda_{k}\right)}\right\} \cdot\left\{\frac{\sinh (\xi-\boldsymbol{\aleph} / N) \sinh (\mathrm{i} \zeta+\xi+\boldsymbol{\aleph} / N)}{\sinh (\xi+\boldsymbol{\aleph} / N) \sinh (\mathrm{i} \zeta-\xi+\boldsymbol{\aleph} / N)}\right\}^{N} .
$$

Obviously, by definition of the Bethe roots, it holds that $\left(\partial_{\xi}^{p} \widehat{\mathfrak{a}}\right)\left(\lambda_{a}\right)=-\delta_{p, 0}$ for $a=1, \ldots, M$ and $p=0, \ldots, k_{\lambda_{a}}-1$ where $k_{\lambda_{a}}$ is the multiplicity of the root $\lambda_{a}$, while $\left(\partial_{\xi}^{k_{\lambda_{a}}} \widehat{\mathfrak{a}}\right)\left(\lambda_{a}\right) \neq 0$. Since

- the total order of $\widehat{a}$ 's poles in a fundamental strip of width $\pi$ is $M+2 N$,

- $\lim _{\mathfrak{R}(\lambda) \rightarrow \pm \infty} \mathfrak{a}(\lambda)=\mathrm{e}^{-\frac{h}{T}+2 \mathrm{i} \zeta(N-M)}$,

the function $1+\widehat{\mathfrak{a}}$ admits $2 N$ more zeroes, counted with multiplicities, in a strip of width $\pi$.

One then picks a domain $\mathcal{D} \subset \mathcal{S}_{\zeta_{\mathrm{m}} / 2}$ such that

- the $N$-fold pole at $-\mathbf{s} / N$ of $\widehat{a}$ is contained in $\mathcal{D}$;

- $1+\widehat{\mathfrak{a}}$ does not vanish on $\partial \mathcal{D}$ and also, has no poles on this boundary;

- $\overline{\mathcal{D}}$ is bounded.

A domain $\mathcal{D}$ being chosen, the auxiliary function $\widehat{\mathfrak{a}}$ associated with the Bethe roots $\left\{\lambda_{a}\right\}_{1}^{M}$ will be such that $1+\widehat{a}$ might have zeroes in $\mathcal{D}$. Some of these zeroes correspond to the subset of $\left\{\lambda_{a}\right\}_{1}^{M}$ contained in $\mathcal{D}$, but there may be other zeroes not corresponding to Bethe roots. These will form the set $\widehat{\mathfrak{X}}$. Namely, one denotes $\widehat{x}_{1}, \ldots, \widehat{x}_{|\mathfrak{x}|}$ the zeroes of $1+\widehat{\mathfrak{a}}$ in $\mathcal{D}$ which do not belong to the set $\left\{\lambda_{a}\right\}_{1}^{M}$ and which are repeated according to their multiplicities. One defines

$$
\widehat{\mathfrak{X}}=\left\{\widehat{x}_{a}\right\}_{1}^{\widehat{|x|}}=\left\{\left(z, k_{z}\right): z \in \mathcal{D} \text { with } z \neq \lambda_{1}, \ldots, \lambda_{M} \text { and } z \text { is a zero of } 1+\widehat{\mathfrak{a}} \text { of order } k_{z}\right\} .
$$


The roots $\lambda_{a}$ which are not contained in $\overline{\mathcal{D}}$ are denoted as $\widehat{y}_{1}, \ldots, \widehat{y}_{|\widehat{y}|}$ and repeated according to their multiplicity. They are gathered in the set

$$
\widehat{\mathcal{y}}=\left\{\widehat{y}_{a}\right\}_{1}^{|\widehat{y}|}=\left\{\left(z, k_{z}\right) \in\left\{\lambda_{a}\right\}_{1}^{M}: z \notin \overline{\mathcal{D}} \bmod \mathrm{i} \pi \mathbb{Z}\right\} .
$$

Since the Bethe roots are admissible, so is the set $\left\{\widehat{y}_{1}, \ldots, \widehat{y}_{\left|\widehat{y}_{\mid}\right|}\right\}$. One further defines the set $\widehat{y}_{\text {sg }}$ as in (4.10). Then the poles of $\widehat{a}$ in $\mathcal{D}$ are located at $y$, with $\left(y, k_{y}\right) \in \widehat{\mathcal{Y}}_{\text {sg }}$. The order of the pole if then given by the multiplicity $k_{y}$ of $y$.

The crucial observation of [5] is that one may express a logarithm $\widehat{\mathscr{A}}$ of $\widehat{\mathfrak{a}}, v i z$. a function $\widehat{\mathscr{A}}$ satisfying to the relation

$$
\widehat{\mathfrak{a}}=\exp \{\widehat{\mathfrak{A}}\},
$$

by means of a direct calculation of residues. Indeed, observe that for any $|\mathfrak{J}(\xi)| \leq \zeta_{\mathrm{m}} / 2$, the map $u \mapsto \theta(\xi-u)$ is holomorphic on $\mathcal{D}$ due to $\mathcal{D} \subset \mathcal{S}_{\zeta_{\mathrm{m}} / 2}$ what ensures that the function's cuts are located away from that domain. By definition of the sets $\widehat{\mathfrak{X}}, \widehat{\mathcal{Y}}$, and $\widehat{\mathcal{Y}}_{\mathrm{sg}}$, this then yields

$$
\widehat{\mathfrak{A}}(\xi)=-\frac{h}{T}+\mathfrak{w}_{N}(\xi)-\mathrm{i} \pi s+\mathrm{i} \sum_{y \in \widehat{\mathbb{Y}}} \theta_{+}(\xi-y)+\mathrm{i} \oint_{\partial \mathcal{D}} \theta(\xi-u) \frac{\widehat{\mathfrak{a}}^{\prime}(u)}{1+\widehat{\mathfrak{a}}(u)} \cdot \frac{\mathrm{d} u}{2 \mathrm{i} \pi} .
$$

Here, the summation set $\widehat{\mathbb{Y}}$ is as defined in (4.16). Finally, if for some $\left(y, k_{y}\right) \in \widehat{\mathbb{Y}}, \xi-y$ lies on a cut of $\theta$, then the formula should be understood in the sense of +boundary values ${ }^{\dagger}$.

Upon using that $\widehat{\mathfrak{A}}$ is a logarithm for $\widehat{\mathfrak{a}}$, one may recast $(4.22$ ) into a non-linear integral equation for $\widehat{\mathfrak{A}}$

$$
\widehat{\mathfrak{A}}(\xi)=-\frac{h}{T}+\mathfrak{w}_{N}(\xi)-\mathrm{i} \pi s+\mathrm{i} \sum_{y \in \widehat{\mathbb{Y}}} \theta(\xi-y)+\mathrm{i} \oint_{\partial \mathcal{D}} \theta(\xi-u) \frac{\widehat{\mathfrak{A}}^{\prime}(u)}{1+\mathrm{e}^{-\widehat{\mathfrak{A}}(u)}} \cdot \frac{\mathrm{d} u}{2 \mathrm{i} \pi} .
$$

It remains to recast (4.23) into its equivalent form given by (4.13). To achieve this, one should first establish the monodromy condition

$$
\oint_{\partial \mathcal{D}} \frac{\widehat{\mathfrak{A}}^{\prime}(u)}{1+\mathrm{e}^{-\widehat{\mathfrak{I}}(u)}} \cdot \frac{\mathrm{d} u}{2 \mathrm{i} \pi}=M-N-|\widehat{\mathcal{Y}}|-\left|\widehat{\mathfrak{Y}}_{\mathrm{sg}}\right|+|\widehat{\mathfrak{X}}|
$$

which is a simple consequence of a residue calculation. Then, an integration by parts yields

$$
\oint_{\partial \mathcal{D}} \theta(\xi-u) \cdot \frac{\widehat{\mathfrak{A}}^{\prime}(u)}{1+\mathrm{e}^{-\widehat{\mathfrak{A}}(u)}} \cdot \frac{\mathrm{d} u}{2 \pi}=\oint_{\partial \mathcal{D}} K(\xi-u) \mathcal{L} \mathrm{n}\left[1+\mathrm{e}^{\widehat{\mathfrak{A}}}\right](u) \cdot \mathrm{d} u-\mathrm{i}(s+|\widehat{\mathbb{Y}}|) \theta(\xi-\kappa) .
$$

This concludes the proof of the first part of the statement.

It remains to prove the second part of the statement, namely that a solution to the non-linear problem gives rise to an admissible solution to the Bethe Ansatz equations. Thus, assume that one is given a solution $(\widehat{\mathfrak{A}}, \overrightarrow{\mathfrak{X}}, \widehat{y})$ to the non-linear problem.

\footnotetext{
$\dagger$ While we make the choice of such a prescription, it is not so relevant in that the choice of any boundary value would still lead to the same definition of $\widehat{a}$.
} 
The very form of the non-linear integral equation satisfied by $\widehat{\mathfrak{A}}$, ensures that $\widehat{\mathfrak{a}}(\xi)=\mathrm{e}^{\widehat{\mathfrak{A}}(\xi)}$ is a meromorphic function on $\mathcal{D}$ that extends to a meromorphic, i $\pi$-periodic function on $\mathbb{C}$ by the formula

$$
\begin{aligned}
& \widehat{\mathfrak{a}}(\xi)=(-1)^{s} \cdot \prod_{\epsilon= \pm}\left[1+\mathrm{e}^{\widehat{\mathfrak{A}}\left(\xi-\epsilon \mathrm{i} \zeta_{\mathrm{m}}\right)}\right]^{\epsilon \operatorname{sgn}(\pi-2 \zeta) \mathbf{1}_{\mathcal{D}}\left(\xi-\epsilon \mathrm{i} \zeta_{\mathrm{m}}\right)} \cdot \prod_{y \in \widehat{\mathbb{Y}}_{\kappa}}\left\{\frac{\sinh (\mathrm{i} \zeta+y-\xi)}{\sinh (\mathrm{i} \zeta+\xi-y)}\right\} \\
&\left(\frac{\sinh (\xi-\boldsymbol{\aleph} / N) \sinh (\xi+\boldsymbol{\aleph} / N-\mathrm{i} \zeta)}{\sinh (\xi+\boldsymbol{\aleph} / N) \sinh (\xi-\boldsymbol{\aleph} / N-\mathrm{i} \zeta)}\right)^{N} \cdot \exp \left\{-\frac{h}{T}+\oint_{\partial \mathcal{D}} K(\xi-u) \cdot \mathcal{L} \mathrm{n}\left[1+\mathrm{e}^{\widehat{\mathfrak{A}}}\right](u) \cdot \mathrm{d} u\right\} .
\end{aligned}
$$

Here, $\mathbf{1}_{A}$ stands for the indicator function of the set $A$. In particular, $1+\widehat{\mathfrak{a}}$ has no poles or zeroes on $\partial \mathcal{D}$ and the only singularities of $\widehat{\mathfrak{a}}$ in $\mathcal{D}$ consist of an $N^{\text {th }}$ order pole at $-\boldsymbol{\kappa} / N$ and poles at $y$ modulo i $\pi \mathbb{Z}$, with $\left(y, k_{y}\right) \in \widehat{\mathcal{Y}}_{\text {sg }}$, whose order corresponds to the multiplicity $k_{y}$ of $y$. Being meromorphic on $\overline{\mathcal{D}}, 1+\widehat{\mathfrak{a}}$ admits a finite number $N_{\mathcal{Z}}$ of zeroes on $\mathcal{D}$, which are repeated according to their multiplicities $\mu_{1}, \ldots, \mu_{N_{Z}}$. Then, by construction, one has $\widehat{\mathfrak{X}} \subset\left\{\mu_{a}\right\}_{1}^{N \mathcal{Z}}$.

Integrating by parts the contour integral in (4.13) with the help of the monodromy condition and, then taking the resulting integral by residues, ensures that, for $\xi \in \mathcal{D}$, it holds

$$
\widehat{\mathfrak{A}}(\xi)=-\frac{h}{T}+\mathfrak{w}_{N}(\xi)-\mathrm{i} \pi s+\mathrm{i} \sum_{y \in \widehat{\mathbb{Y}}} \theta_{+}(\xi-y)-\mathrm{i} N \theta\left(\xi+\frac{\aleph}{N}\right)-\mathrm{i} \sum_{y \in \widehat{\mathscr{Y}}_{\mathrm{sg}}} \theta(\xi-y)+\mathrm{i} \sum_{a=1}^{N_{\mathcal{Z}}} \theta\left(\xi-\mu_{a}\right)
$$

The set $\left\{\mu_{a}\right\}_{1}^{N \mathcal{Z}} \oplus \widehat{\mathcal{Y}} \ominus \widehat{\mathfrak{X}}$ unambiguously defines the set $\left\{\lambda_{a}\right\}_{1}^{M}$ built up from $M$ complex numbers $\lambda_{1}, \ldots, \lambda_{M}$. The cardinality $M$ of this set can be related to the parameter $s$ by taking explicitly the monodromy condition, leading to the constraint $s=N-M$. Then, the above ensures that $\widehat{\mathfrak{a}}$ is expressed in terms of $\lambda_{1}, \ldots, \lambda_{M}$ exactly as given in (4.18). Furthermore, it holds that $\left(\partial_{\xi}^{p} \widehat{\mathfrak{a}}\right)\left(\lambda_{a}\right)=-\delta_{p, 0}$ for $a=1, \ldots, M$ and $p=0, \ldots, k_{\lambda_{a}}-1$, where $k_{\lambda_{a}}$ is the multiplicity of the root $\lambda_{a}$ and that $\left(\partial_{\xi}^{k_{d}} \widehat{\mathfrak{a}}\right)\left(\lambda_{a}\right) \neq 0$. Thus, the $\lambda_{a}$ 's satisfy the system of Bethe Ansatz equations.

It remains to establish that the roots $\lambda_{1}, \ldots, \lambda_{M}$ so constructed are admissible. Since $\left\{\mu_{a}\right\}_{1}^{N_{\mathcal{Z}}} \subset \mathcal{D} \subset \mathcal{S}_{\zeta_{\mathrm{m}} / 2}$, the roots $\mu_{a}$ are mutually admissible. Also, by the properties of solutions to the non-linear problem, so are the roots $\widehat{y}$. Thus, the only possibility for $\left\{\lambda_{a}\right\}_{1}^{M}$ to be a non-admissible solution set is that there exists a root $\mu_{a}$ such that $\mu_{a}=y \pm \mathrm{i} \zeta_{\mathrm{m}}$. Since the set $\widehat{\mathcal{Y}}_{\mathrm{sg}}$ gathers the poles of $\widehat{\mathfrak{a}}$ inside of $\mathcal{D}$, which obviously cannot give rise to zeroes of $1+\widehat{\mathfrak{a}}$, the only possible solution is that $\mu_{a}=\lambda_{b}+\operatorname{isgn}(\pi-2 \zeta) \zeta_{\mathrm{m}}$, for some $\left(\lambda_{b}, k_{\lambda_{b}}\right) \in \widehat{\mathcal{y}}$. However, one has that

$$
\begin{aligned}
\widehat{\mathfrak{a}}\left(\lambda_{b}+\operatorname{isgn}(\pi-2 \zeta) \zeta_{\mathrm{m}}+\epsilon\right)=\frac{(-1)^{s} \mathrm{e}^{-\frac{h}{T}}}{\sinh (-\epsilon)} \cdot \prod_{\substack{k=1 \\
\neq b h}}^{M}\left\{\frac{\sinh \left(\lambda_{k}-\lambda_{b}-\epsilon\right)}{\sinh \left(2 \mathrm{i} \zeta+\lambda_{b}-\lambda_{k}+\epsilon\right)}\right\} \\
\times\left(\frac{\sinh \left(\lambda_{b}-\boldsymbol{\aleph} / N+\mathrm{i} \zeta+\epsilon\right) \sinh \left(2 \mathrm{i} \zeta+\lambda_{b}+\boldsymbol{\aleph} / N+\epsilon\right)}{\sinh \left(\lambda_{b}+\boldsymbol{\aleph} / N+\mathrm{i} \zeta+\epsilon\right) \sinh \left(-\lambda_{b}+\boldsymbol{\aleph} / N-\epsilon\right)}\right)^{N}
\end{aligned}
$$

In order for the $\epsilon \rightarrow 0$ limit not to give zero, one needs the $\sinh (\epsilon)$ prefactor to be compensated by some $\epsilon^{-1}$ singularity which would stem from the remaining factors. By definition of $\widehat{y}$ being admissible, the last term cannot blow up in the $\epsilon \rightarrow 0$ limit. Hence, the only possibility is that $2 \mathrm{i} \zeta+\lambda_{b}-\lambda_{k}=0$ for some $k$. This however means that $\mu_{a}=\lambda_{k}-\operatorname{isgn}(\pi-2 \zeta) \zeta_{\mathrm{m}}$, what is a contradiction to the previous argument.

It turns out that the knowledge of the $\widehat{\mathfrak{A}}$ function allows one to compute all the quantities of interest in the model. For instance, one may recast the Eigenvalue of the transfer matrix associated with a distribution of roots 
$\left\{\lambda_{a}\right\}_{a=1}^{M}$ in terms of the associated function $\widehat{\mathfrak{A}}$ as

$$
\begin{aligned}
\tau\left(0 \mid\left\{\lambda_{a}\right\}_{a=1}^{M}\right)=\prod_{y \in \widehat{\mathbb{Y}}_{k}}\left\{\frac{\sinh (y-\mathrm{i} \zeta)}{\sinh (y)}\right\} \cdot\left(\frac{\sinh (\boldsymbol{\aleph} / N+\mathrm{i} \zeta)}{\sinh (\mathrm{i} \zeta)}\right)^{2 N} & \\
& \quad \times \exp \left\{\frac{h}{2 T}-\oint_{\partial \mathcal{D}} \frac{\sin (\zeta) \mathcal{L}\left[1+\mathrm{e}^{\widehat{\mathscr{A}}}\right](u)}{\sinh (u-\mathrm{i} \zeta) \sinh (u)} \cdot \frac{\mathrm{d} u}{2 \pi}\right\}
\end{aligned}
$$

See e.g. [5] for the details of the algebraic manipulations. Also, in (4.29), we employed the convention for products which was introduced in (1.25). Finally, by changing the integration point from $\kappa$ to $\kappa^{\prime}$ in the definition of $\ln \left[1+\mathrm{e}^{\widehat{\mathfrak{A}}}\right]$ one may readily check that (4.29) does not depend on the choice of $\kappa$, as it should be.

It is clear from the proof that for a given set of admissible Bethe roots $\left\{\lambda_{a}\right\}_{1}^{M}$, there is a very large choice of domains $\mathcal{D}$ and that different choices of domains $\mathcal{D}$ may lead to very different sets $\widehat{\mathfrak{X}}$ and $\widehat{\mathcal{Y}}$. Thus, apparently very different non-linear integral equations may lead to exactly the same solution to the Bethe Ansatz equations. Thus, in order to hope finding all distinct solutions to the original problem of solving Bethe equations, a reasonable strategy seems to fix, once and for all, the domain $\mathcal{D}$ and to look for different solutions giving rise to distinct sets $\widehat{\mathfrak{X}}$ and $\widehat{\mathcal{Y}}$. Such a construction is also dictated by the physical content of the non-linear integral equation.

As shown earlier on in Proposition 3.1, $t_{q}$ admits a non-degenerate dominant Eigenvalues $\widehat{\Lambda}_{\text {max }}$ : all the other Eigenvalues are smaller in modulus. It was argued in the literature [5,6,18,21] and this property will be established rigorously in the following, that this dominant Eigenvalue is described by a solution set $\left\{\lambda_{a}^{(\max )}\right\}_{1}^{N}$ to the Bethe equations containing exactly $N$ distinct admissible Bethe roots, $\lambda_{1}^{(\max )}, \ldots, \lambda_{N}^{(\max )}$.

Let $\widehat{\mathfrak{a}}_{\max }$ stand for the auxiliary function associated with this distribution of roots. One then considers a domain $\mathcal{D}$ along with its canonically oriented boundary $\partial \mathcal{D}$ such that

- $\left\{\lambda_{a}^{(\max )}\right\}_{1}^{M} \subset \mathcal{D}$;

- no other zero of $1+\widehat{\mathfrak{a}}_{\max }$, viz. one that would not be a Bethe root, is contained in $\overline{\mathcal{D}}$;

- the $N$-fold pole at $-\boldsymbol{\aleph} / N$ is contained in $\mathcal{D}$;

- no other pole of $\widehat{\mathfrak{a}}_{\text {max }}$, is contained in $\overline{\mathcal{D}}$.

Note that there is still a rather large freedom of choice of the domain $\mathcal{D}$, although for practical purposes, some may turn out to be more suited than others. Traditionally, owing to its important physical interpretation, this domain $\mathcal{D}$ is then used to study the non-linear problem involving non-empty sets $\widehat{\mathfrak{X}}$ and $\widehat{\mathcal{Y}}$ for which the associated solution, if existing, will give rise to some Eigenvalue of the quantum transfer matrix differing from the dominant one.

The domain $\mathcal{D}$ so chosen for the dominant state's Bethe roots could, in principle, be located outside of $\mathcal{S}_{\zeta_{\mathrm{m}} / 2}$. However, as will be shown in a later stage of this work, for temperature high enough, it is not so and, in fact, $\mathcal{D}$ can be taken as a small disc centred at the origin.

The domain $\mathcal{D}$ associated with the dominant Eigenvalue's Bethe roots being fixed, the sets $\widehat{\mathfrak{X}}$ and $\widehat{\mathscr{Y}}$ subordinate to it are usually called the hole set -for $\widehat{\mathfrak{X}}$ - and the particle set -for $\widehat{\mathcal{Y}}$ -

\subsection{The formal infinite Trotter number limit}

The main advantage of the non-linear integral equation based description, relies on the possibility to easily take, at least on formal grounds, the Trotter limit on the level of the integral representation for the Eigenvalues (4.29) and for the solution to the non-linear integral equations (4.13) driving these. Indeed, the parameter $N$ only appears 
in the driving term $\mathfrak{w}_{N}$ of (4.13). Thus, assuming that $\widehat{\mathfrak{A}} \underset{N \rightarrow+\infty}{\longrightarrow} \mathfrak{A}$ pointwise on $\partial \mathcal{D}$, and that all properties of the non-linear problem are preserved under this limit, one may readily characterise the limit of $\tau\left(0 \mid\left\{\lambda_{a}\right\}_{a=1}^{M}\right)$ as well as derive a non-linear integral equation satisfied by $\mathfrak{A}$. Indeed, in the infinite Trotter number limit, one has

$$
\mathfrak{w}_{N}(\xi) \underset{N \rightarrow+\infty}{\longrightarrow}-2 \boldsymbol{N}(\operatorname{coth}(\xi)-\operatorname{coth}(\xi-\mathrm{i} \zeta))=\frac{2 J \sin ^{2}(\zeta)}{T \sinh (\xi) \sinh (\xi-\mathrm{i} \zeta)}
$$

In addition one assumes the existence of the limit of the particle and hole roots

$$
\widehat{x}_{a} \rightarrow x_{a}, a=1, \ldots,|\mathfrak{x}| \quad \text { and } \quad \widehat{y}_{a} \rightarrow y_{a}, a=1, \ldots,|y|
$$

All these assumptions result in the non-linear integral equation satisfied by the limit function on $\mathcal{S}_{\zeta_{\mathrm{m}} / 2}$ :

$$
\mathfrak{U}(\xi)=-\frac{1}{T} e_{0}(\xi)-\mathrm{i} \pi s+\mathrm{i} \sum_{y \in \mathbb{Y}_{K}} \theta(\xi-y)+\oint_{\partial \mathcal{D}} K(\xi-u) \cdot \mathcal{L}\left[1+\mathrm{e}^{\mathfrak{A}}\right](u) \cdot \mathrm{d} u
$$

Here,

$$
e_{0}(\xi)=h-\frac{2 J \sin ^{2}(\zeta)}{\sinh (\xi) \sinh (\xi-\mathrm{i} \zeta)} .
$$

Furthermore,

$$
\mathbb{Y}_{\kappa}=\mathbb{Y} \ominus\{\kappa\}^{\oplus(s+|\mathbb{Y}|)} \text { with } \quad \mathbb{Y}=\mathcal{Y} \oplus \mathcal{Y}_{\text {sg }} \ominus \mathfrak{X},
$$

and where

$$
\mathfrak{X}=\left\{x_{a}\right\}_{a=1}^{|\mathfrak{F}|} \quad \text { and } \quad \boldsymbol{Y}=\left\{y_{a}\right\}_{a=1}^{|\mathcal{Y}|} .
$$

The non-linear integral equation at infinite Trotter number is to be supplemented with the constraints

$$
\oint_{\partial \mathcal{D}} \frac{\mathfrak{H}^{\prime}(u)}{1+\mathrm{e}^{-\mathfrak{H}(u)}} \cdot \frac{\mathrm{d} u}{2 \mathrm{i} \pi}=|\mathfrak{X}|-|\boldsymbol{Y}|-\left|\boldsymbol{Y}_{\mathrm{sg}}\right|-s,
$$

and

$$
\left\{\begin{array}{lll}
\left\{\partial_{\xi}^{r} \mathrm{e}^{\mathfrak{I}(\xi)}\right\}_{\mid \xi=x}=-1 & \text { for } \quad\left(x, k_{x}\right) \in \mathfrak{X}, & r=0, \ldots, k_{x}-1, \\
\left\{\partial_{\xi}^{r} \mathrm{e}^{\mathfrak{I}(\xi)}\right\}_{\mid \xi=y}=-1 & \text { for } \quad\left(y, p_{y}\right) \in \mathcal{Y}, & r=0, \ldots, p_{y}-1
\end{array}\right.
$$

where $k_{x}$, resp. $p_{y}$, is the multiplicity of $x$ in respect to the roots $x_{1}, \ldots, x_{|\mathfrak{x}|}$, resp. of $y$ in respect to the roots $y_{1}, \ldots, y_{|y|}$.

Similar handlings lead to

$$
\begin{aligned}
\lim _{N \rightarrow+\infty}\left\{\tau\left(0 \mid\left\{\lambda_{a}\right\}_{a=1}^{M}\right)\right\}= & \prod_{y \in \mathbb{Y}_{k}}\left\{\frac{\sinh (y-\mathrm{i} \zeta)}{\sinh (y)}\right\} \\
& \quad \times \exp \left\{\frac{h}{2 T}-\frac{2 J}{T} \cos (\zeta)-\oint_{\partial \mathcal{D}} \frac{\sin (\zeta) \mathcal{L} \mathrm{n}\left[1+\mathrm{e}^{\mathfrak{I}}\right](u)}{\sinh (u-\mathrm{i} \zeta) \sinh (u)} \cdot \frac{\mathrm{d} u}{2 \pi}\right\} .
\end{aligned}
$$




\section{Existence and uniqueness of solutions to the NLIE for large T}

Recall the notations $\mathcal{S}_{\alpha}$ for the strip of width $\alpha$ centred around $\mathbb{R}$ and $\mathcal{D}_{z_{0}, \alpha}$ for the disc of radius $\alpha$ centred at $z_{0} \in \mathbb{C}$, both introduced in (1.20). In this section, we develop a rigorous framework allowing one to lay a firm ground to the heuristics outlined in the previous section.

\subsection{An auxiliary problem grasping the leading high-temperature behaviour}

The first step of the proof consists in providing a characterisation of the properties of an auxiliary function which will subsequently be shown to grasp the leading large- $T$ asymptotic behaviour of $\mathfrak{A}$.

For further convenience it appears useful to single out a specific class of "particle"-"hole" sets.

Definition 5.1. The sets $\mathfrak{X}, \mathcal{Y}$ are said to belong to the class $C_{\alpha, \varrho}^{\epsilon}$ with cardinalities $n_{x}, n_{y}$ if

- $\mathfrak{X}=\left\{x_{1}, \ldots, x_{|\mathfrak{X}|}\right\} \subset \mathcal{D}_{0, \epsilon}$ with $|\mathfrak{X}|=n_{x}$ and all $x_{a}$ 's are pairwise distinct;

- $\mathcal{Y} \subset \mathcal{S}_{\frac{\pi}{2}} \backslash\left\{\mathcal{D}_{0, \epsilon} \cup_{v= \pm} \mathcal{D}_{v \mathrm{i} \zeta_{\mathrm{m}}, \alpha}\right\}$ and $\boldsymbol{Y}=\left\{y_{1}, \ldots, y_{\left|\mathcal{Y}_{\mid}\right|}\right\}$with $|\mathcal{Y}|=n_{y}$ and the $y_{a}$ 's being pairwise distinct;

- the elements of $y$ are subject to the constraint

$$
\left|(-1)^{s} \prod_{y \in \mathcal{Y}} \frac{\sinh (\mathrm{i} \zeta+y)}{\sinh (\mathrm{i} \zeta-y)}+1\right|>\varrho .
$$

Note that for $\epsilon<\alpha$, all sets $\mathfrak{X}$ and $\mathcal{Y}$ in the class $\mathcal{C}_{\alpha, \varrho}^{\epsilon}$ are such that there are no singular roots $\mathcal{Y}_{\text {sg }}$ relative to $\mathcal{D}_{0, \epsilon}$, viz. $y-\mathrm{i} \zeta_{\mathrm{m}} \notin \mathcal{D}_{0, \epsilon}$ modulo $\mathrm{i} \pi \mathbb{Z}$, for any $y \in \mathcal{Y}$.

Lemma 5.1. Fix integers $n_{x}, n_{y} \geq 0$ and $n_{\kappa} \in \mathbb{Z}$. Let $\alpha, \varrho>0$ be given. Then, there exists $\epsilon>0, \alpha / 2>\epsilon>0$ such that

- for any $\mathfrak{X}, \mathcal{Y}$ in the class $C_{\alpha, \varrho}^{\epsilon}$ with cardinalities $n_{x}, n_{y}$,

- for any $\kappa \in \overline{\mathcal{D}}_{0, \epsilon}$,

the function $1+f$ with

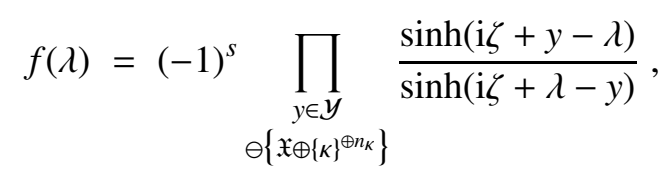

has no zeroes inside of $\overline{\mathcal{D}}_{0, \epsilon}$ and, uniformly in $\overline{\mathcal{D}}_{0, \epsilon}$, is subject to the lower bound

$$
|1+f(\lambda)| \geq \frac{\varrho}{2}
$$

Proof. On may recast

$$
1+f(\lambda)=\left((-1)^{s} \prod_{y \in \mathcal{Y}} \frac{\sinh (\mathrm{i} \zeta+y)}{\sinh (\mathrm{i} \zeta-y)}+1\right) \mathrm{e}^{u(\lambda)}+1-\mathrm{e}^{u(\lambda)}
$$

with

$$
u(\lambda)=\mathrm{i} \sum_{y \in \mathcal{Y}}[\widetilde{\theta}(\lambda-y)-\widetilde{\theta}(-y)]-\mathrm{i} \sum_{y \in \mathfrak{X} \oplus\{\kappa\}^{n_{\kappa}}} \widetilde{\theta}(\lambda-y) .
$$


Here, $\widetilde{\theta}$ corresponds to a choice of the branch of the logarithm defining $\theta$ in such a way that $\lambda \mapsto \widetilde{\theta}(\lambda-y)$ is holomorphic on $\mathcal{D}_{0, \epsilon}$. Note that the branch may differ for different choices of $y \in \mathcal{Y}$. We point out that such a choice is possible since singular roots do not exist due to $\mathcal{Y} \subset \mathscr{B}_{\alpha, \epsilon}$ with

$$
\mathscr{B}_{\alpha, \epsilon}=\mathcal{S}_{\frac{\pi}{2}} \backslash\left\{\mathcal{D}_{0, \epsilon} \cup_{v= \pm} \mathcal{D}_{v \mathrm{i} \zeta_{\mathrm{m}}, \alpha}\right\}
$$

for $\epsilon>0$ small enough. Observe that if $0<\epsilon<\alpha / 2$ and $\alpha$ is small enough, then for any $\lambda \in \mathcal{D}_{0, \epsilon}$,

$$
y \in \mathscr{B}_{\alpha, \epsilon} \Rightarrow \lambda-y \in \mathcal{S}_{\frac{\pi}{2}+\epsilon} \backslash \underset{v= \pm}{\cup} \mathcal{D}_{v \mathrm{i} \zeta_{\mathrm{m}}, \alpha-\epsilon} .
$$

Thus, by the mean value theorem and $\mathrm{i} \pi$ - periodicity of $K$,

$$
\begin{aligned}
|u(\lambda)| & \leq \epsilon|\mathcal{Y}| \sup _{s \in \mathcal{D}_{0, \epsilon}} \sup _{w \in \mathscr{B}_{\alpha, \epsilon}}|2 \pi K(s-w)|+2 \epsilon\left(|\mathfrak{X}|+\left|n_{\kappa}\right|\right) \sup _{s \in \mathcal{D}_{0,2 \epsilon}}|2 \pi K(s)| \\
& \leq 4 \pi \epsilon\left(|\mathcal{Y}|+|\mathfrak{X}|+\left|n_{\kappa}\right|\right) \sup _{w \in \mathscr{B} \frac{\alpha}{2}, 0}|K(w)| .
\end{aligned}
$$

The above bounds ensure the existence of an $\epsilon$-independent constant $C>0$ such that

$$
|1+f(\lambda)| \geq\left|\rho \mathrm{e}^{-|u(\lambda)|}-\right| u(\lambda)\left|\mathrm{e}^{|u(\lambda)|}\right| \geq \rho \mathrm{e}^{-\epsilon C}-\epsilon C \mathrm{e}^{\epsilon C} \geq \frac{\varrho}{2}
$$

provided that $\epsilon$ is small enough.

The above lemma has an immediate corollary.

Corollary 5.2. For given $n_{x}, n_{y} \in \mathbb{N}$ there exists $\epsilon>0$ such that for any $\mathfrak{X}$ and $\mathcal{Y}$ in the class $C_{\alpha, \varrho}^{\epsilon}$ with cardinalities $n_{x}, n_{y}$ and, for any $\kappa \in \partial \mathcal{D}_{0, \epsilon}$, upon denoting

$$
\mathbb{Y}_{\kappa}=\mathcal{Y} \ominus\left\{\mathfrak{X} \oplus\{\kappa\}^{\oplus s+|y|+\left|y_{\mathrm{sg}}\right|-|\mathfrak{X}|}\right\},
$$

the function

$$
\mathfrak{A}_{\infty}(\xi)=-\mathrm{i} \pi s+\mathrm{i} \sum_{y \in \mathbb{Y}_{\kappa}} \theta(\xi-y)
$$

has no zeroes in $\overline{\mathcal{D}}_{0, \epsilon}$, satisfies the lower bound

$$
\left|1+\mathrm{e}^{\mathfrak{H}_{\infty}(\lambda)}\right| \geq \frac{\varrho}{2} \quad \text { on } \quad \overline{\mathcal{D}}_{0, \epsilon}
$$

and solves the non-linear integral equation

$$
\mathfrak{A}_{\infty}(\xi)=-\mathrm{i} \pi s+\mathrm{i} \sum_{y \in \mathbb{Y}_{\kappa}} \theta(\xi-y)+\oint_{\partial \mathcal{D}_{0, \epsilon}} K(\xi-u) \mathcal{L} \mathrm{n}\left[1+\mathrm{e}^{\mathfrak{A}_{\infty}}\right](u) \cdot \mathrm{d} u,
$$

for $\xi \in \mathcal{S}_{\zeta_{\mathrm{m}} / 2}$, where the logarithm is defined as

$$
\mathcal{L}\left[1+\mathrm{e}^{\mathfrak{H}_{\infty}}\right](u)=\int_{\kappa}^{u} \frac{\mathfrak{H}_{\infty}^{\prime}(s)}{1+\mathrm{e}^{-\mathfrak{H}_{\infty}(s)}} \cdot \mathrm{d} s+\ln \left[1+\mathrm{e}^{\mathfrak{H}_{\infty}(\kappa)}\right]
$$

and the integral runs along $\partial \mathcal{D}_{0, \epsilon}$, from $\kappa$ to $u$.

Finally, $\mathfrak{H}_{\infty}$ satisfies the zero-monodromy condition

$$
\oint_{\partial \mathcal{D}_{0, \epsilon}} \frac{\mathfrak{H}_{\infty}^{\prime}(s)}{1+\mathrm{e}^{-\mathfrak{H}_{\infty}(s)}} \cdot \frac{\mathrm{d} s}{2 \mathrm{i} \pi}=0 .
$$


Note that, (5.14) is well-defined since the condition (5.13) ensures that $\mathcal{L} n\left[1+\mathrm{e}^{\mathfrak{H}_{\infty}}\right]$ can be extended to an analytic function on $\overline{\mathcal{D}}_{0, \epsilon}$; in particular, the result does not depend on the path used for the continuation. Also, we recall that due to $\mathcal{Y} \subset \mathcal{S}_{\frac{\pi}{2}} \backslash\left\{\mathcal{D}_{0, \epsilon} \cup_{v= \pm} \mathcal{D}_{v i \zeta_{\mathrm{m}}, \alpha}\right\}$, one has that $\mathcal{Y}_{\mathrm{sg}}=\emptyset$ and hence the definitions of $\mathbb{Y}_{\kappa}$ given in (5.11) and (4.34) are consistent.

\section{Proof-}

The zero-monodromy condition follows from the fact that $\mathfrak{A}_{\infty}$ is holomorphic on $\mathcal{D}_{0, \epsilon}$ and that one has the lower bound (5.13).

Using that $K(\xi-u)$ is meromorphic in $\mathcal{S}_{\frac{\pi}{2}}$ with simple poles at $u=\xi \pm \mathrm{i} \zeta_{\mathrm{m}} \bmod \mathrm{i} \pi \mathrm{n}$ we conclude by means of the residue theorem that, for $\xi \in \mathcal{S}_{\zeta_{\mathrm{m}} / 2}$

$$
\oint_{\partial \mathcal{D}_{0, \epsilon}} K(\xi-u) \mathcal{L} \mathrm{n}\left[1+\mathrm{e}^{\mathfrak{I}_{\infty}}\right](u) \cdot \mathrm{d} u=0
$$

\subsection{An auxiliary fixed point problem}

Recall the definition (5.12) of the function $\mathfrak{A}_{\infty}(\xi)$, and introduce

$$
\mathscr{L}(v, x)=\frac{1}{1+\mathrm{e}^{-\mathfrak{H}_{\infty}(v)-x}} .
$$

Further set

$$
\varpi_{N}(\lambda)=h-T \mathfrak{w}_{N}(\lambda)
$$

and, given $\epsilon>0$, define

$$
\chi_{N ; \epsilon}(\lambda)=-\oint_{\partial \mathcal{D}_{0, \epsilon}} \frac{K(\lambda-u)}{1+\mathrm{e}^{-\mathfrak{H}_{\infty}(u)}} \cdot \varpi_{N}(u) \cdot \mathrm{d} u .
$$

Next, given $\xi \in O\left(\mathcal{S}_{\zeta_{\mathrm{m}} / 2}\right)$, denote

$$
G[\gamma](v, t)=\gamma(v) \gamma^{\prime}(v) \partial_{2} \mathscr{L}\left(v, \frac{t \gamma(v)}{T}\right)+(1-t) \gamma^{2}(v) \mathfrak{H}_{\infty}^{\prime}(v) \partial_{2}^{2} \mathscr{L}\left(v, \frac{t \gamma(v)}{T}\right) .
$$

Finally, given $\gamma \in O\left(\mathcal{S}_{\zeta_{\mathrm{m}} / 2}\right)$, define the map $0_{T, N}$

$$
0_{T, N}[\xi](\lambda)=\chi_{N ; \epsilon}(\lambda)+\frac{1}{T} \oint_{\partial \mathcal{D}_{0, \epsilon}} \mathrm{d} u K(\lambda-u) \int_{\kappa}^{u} \mathrm{~d} v \int_{0}^{1} \mathrm{~d} t G\left[\xi-\varpi_{N}\right](v, t) .
$$

Above, the $v$ integral runs along $\partial \mathcal{D}_{0, \epsilon}$.

We are going to establish that the operator $0_{T, N}$

- is well-defined on an appropriate functional space, provided $T$ and $N$ are large enough;

- is closely related to the problem of interest, in that it provides one with a rewriting of the non linear integral equation (4.13). 
We first introduce the appropriate functional space. Given $r>0$, set

$$
\mathcal{B}_{r}=\left\{\xi \in O\left(\mathcal{S}_{\zeta_{\mathrm{m}} / 2}\right):\|\xi\|_{L^{\infty}\left(\mathcal{S}_{\zeta_{\mathrm{m}} / 2}\right)} \leq r\right\} \quad \text { with } \quad\|g\|_{L^{\infty}(U)}=\operatorname{supess}_{u \in U}|g(u)| .
$$

By virtue of Montel's theorem, for any $r>0, \mathcal{B}_{r}$ is a complete metric space with respect to the distance

$$
d(f, g)=\|f-g\|_{L^{\infty}\left(\mathcal{S}_{\zeta \mathrm{m} / 2}\right)} .
$$

In the following set

$$
\mathfrak{c}=2\left\|\chi_{\infty ; \epsilon}\right\|_{L^{\infty}\left(\mathcal{S}_{\zeta \mathrm{m} / 2}\right)} .
$$

Proposition 5.3. For given $n_{x}, n_{y} \in \mathbb{N}$ there exist $T_{0}>0, N_{0} \in \mathbb{N}$ and $\epsilon>0$ such that for any $\mathfrak{X}$ and $\mathcal{Y}$ in the class $C_{\alpha, \varrho}^{\epsilon}$ with cardinalities $n_{x}, n_{y}$ and for any $\kappa \in \partial \mathcal{D}_{0, \epsilon}$

(i) $O_{T, N}$ is well-defined on $\mathcal{B}_{\mathfrak{c}}$, with $\mathrm{c}$ as given in (5.25);

(ii) $O_{T, N}$ stabilises $\mathcal{B}_{\mathfrak{c}}$, viz. $O_{T, N}\left[\mathcal{B}_{\mathrm{c}}\right] \subset \mathcal{B}_{\mathrm{c}}$;

(iii) the solutions to the non-linear integral equation (4.13) associated with the sets $\mathcal{Y}$ and $\mathfrak{X}$ are in one-to-one correspondence with the fixed points of the operator $O_{T, N}$.

\section{Proof-}

We start by establishing (iii). Upon making the change of unknown function

$$
\widehat{\mathfrak{A}}(\lambda)=\frac{1}{T}\left(\xi(\lambda)-\varpi_{N}(\lambda)\right)+\mathfrak{A}_{\infty}(\lambda),
$$

where $\mathfrak{A}_{\infty}(\lambda)$ was introduced in $(5.12)$, one recasts the NLIE (4.13) in the form

$$
\xi(\lambda)=T \oint_{\partial \mathcal{D}_{0, \epsilon}} K(\lambda-u) \cdot\left\{\mathcal{L} \mathrm{n}\left[1+\mathrm{e}^{\mathfrak{A}_{\infty}+\frac{\xi-\omega_{N}}{T}}\right](u)-\mathcal{L} \mathrm{n}\left[1+\mathrm{e}^{\mathfrak{H}_{\infty}}\right](u)\right\}
$$

defining $\xi(\lambda)$ not only on $\partial \mathcal{D}_{0, \epsilon}$ but for all $\lambda \in \mathcal{S}_{\zeta_{\mathrm{m}} / 2}$. Then, the Taylor-integral expansion

$$
\mathscr{L}(v, x)=\mathscr{L}(v, 0)+x \partial_{2} \mathscr{L}(v, 0)+x^{2} \int_{0}^{1}(1-t) \partial_{2}^{2} \mathscr{L}(v, t x) \cdot \mathrm{d} t
$$

leads to the expression

$$
\begin{aligned}
& \mathcal{L}\left[1+\mathrm{e}^{\mathfrak{H}_{\infty}+\frac{\gamma}{T}}\right](u)=\mathcal{L} \mathrm{n}\left[1+\mathrm{e}^{\mathfrak{H}_{\infty}}\right](u)+\frac{1}{T}\left[\frac{\gamma(u)}{1+\mathrm{e}^{-\mathfrak{H}_{\infty}(u)}}-\frac{\gamma(\kappa)}{1+\mathrm{e}^{-\mathfrak{H}_{\infty}(\kappa)}}\right] \\
& +\frac{1}{T^{2}} \int_{\kappa}^{u} \mathrm{~d} v \int_{0}^{1} \mathrm{~d} t\left\{\gamma^{\prime}(v) \gamma(v) \partial_{2} \mathscr{L}\left(v, \frac{t \gamma(v)}{T}\right)+(1-t) \gamma^{2}(v) \mathfrak{A}_{\infty}^{\prime}(v) \partial_{2}^{2} \mathscr{L}\left(v, \frac{t \gamma(v)}{T}\right)\right\} .
\end{aligned}
$$

Altogether, this entails that $\xi$ is a fixed point of $0_{T, N}$ :

$$
\xi(\lambda)=0_{T, N}[\xi](\lambda) .
$$


By tracing the above steps backwards, one concludes that any fixed point $\xi$ of $0_{T, N}$ gives rise to a solution $\widehat{\mathfrak{A}}$ of (4.13).

The first claim of the proposition follows once a few preparatory bounds are set. As shown in Lemma 5.1, uniformly in the parameters forming the sets $\mathcal{Y}, \mathfrak{X}$, it holds that

$$
\left|1+\mathrm{e}^{\mathfrak{H}_{\infty}(v)}\right| \geq \frac{\varrho}{2} \quad \text { and } \quad\left|\mathrm{e}^{\mathfrak{H}_{\infty}(v)}\right| \leq C
$$

for any $v \in \mathcal{D}_{0, \epsilon}$, provided that $\epsilon>0$ is small enough. Then, there exists $x_{0}$ such that for any $|x| \leq 2 x_{0}$, one has $\left|1-\mathrm{e}^{-x}\right| \leq \varrho / 4$. Thence, for any $|x| \leq 2 x_{0}$ and $v \in \mathcal{D}_{0, \epsilon}$ one has the lower bound

$$
\left|1+\mathrm{e}^{-x-\mathscr{H}_{\infty}(v)}\right| \geq \frac{\varrho}{4}\left|\mathrm{e}^{-\mathscr{U}_{\infty}(v)}\right| \quad \text { leading to } \quad|\mathscr{L}(v, x)| \leq \frac{4 C}{\varrho} .
$$

Since $x \mapsto \mathscr{L}(v, x)$ is holomorphic on $\mathcal{D}_{0,2 x_{0}}$ it follows that

$$
\left\|\partial_{2}^{k} \mathscr{L}(v, \cdot)\right\|_{L^{\infty}\left(\mathcal{D}_{0, x_{0}}\right)} \leq C_{k}\|\mathscr{L}(v, \cdot)\|_{L^{\infty}\left(\partial \mathcal{D}_{0,2 x_{0}}\right)} \leq \widetilde{C}_{k}
$$

for some constants $C_{k}, \widetilde{C}_{k}$ uniformly in $v \in \mathcal{D}_{0, \epsilon}$.

Let $\xi \in \mathcal{B}_{\mathrm{c}}$ and define

$$
\gamma_{\xi}=\xi-\varpi_{N} .
$$

Then there exists $N_{0} \geq 0$ such that for all $N \geq N_{0}$ the function $\varpi_{N}$ is holomorphic in an annulus containing $\partial \mathcal{D}_{0, \epsilon}$ and $\partial \mathcal{D}_{0,2 \epsilon}$. Thus, for $N \geq N_{0}$, there are constants $C_{k}^{\prime}$ such that

$$
\left\|\gamma_{\xi}^{(k)}\right\|_{L^{\infty}\left(\partial \mathcal{D}_{0, \epsilon}\right)} \leq C_{k}^{\prime}\left\|\gamma_{\xi}\right\|_{L^{\infty}\left(\partial \mathcal{D}_{0,2 \epsilon}\right)} \leq C_{k}^{\prime}\left(\mathfrak{c}+\left\|\varpi_{N}\right\|_{L^{\infty}\left(\partial \mathcal{D}_{0,2 \epsilon}\right)}\right)
$$

where $\gamma_{\xi}^{(0)}=\gamma_{\xi}$ and $\gamma_{\xi}^{(k)}$ for $k>0$ stands for the $\mathrm{k}^{\text {th }}$ derivative of $\gamma_{\xi}$. Due to (5.35) there exists $T_{0}$ such that $T^{-1} \cdot\left\|\gamma_{\xi}\right\|_{L^{\infty}\left(\partial \mathcal{D}_{0, \epsilon}\right)} \leq x_{0}$ for all $T>T_{0}$. Using (5.21), (5.22), (5.33) and (5.35), one establishes the bound

$$
\begin{aligned}
& \left\|0_{T, N}[\xi]\right\|_{L^{\infty}\left(\mathcal{S}_{\zeta \mathrm{m} / 2)} \leq\right.} \leq\left\|\chi_{N ; \epsilon}\right\|_{L^{\infty}\left(\mathcal{S}_{\zeta \mathrm{m} / 2)}\right.} \\
& \quad+\frac{4 \pi^{2} \epsilon^{2}}{T} \cdot\|K\|_{L^{\infty}\left(\mathcal{S}_{\zeta \mathrm{m} / 2+\epsilon}\right)} \cdot C_{0}^{\prime}\left(\mathfrak{c}+\left\|\varpi_{N}\right\|_{L^{\infty}\left(\partial \mathcal{D}_{0,2 \epsilon}\right)}\right)^{2} \cdot\left\{C_{1}^{\prime} \widetilde{C}_{1}+\frac{C_{0}^{\prime} \widetilde{C}_{2}}{2} \cdot\left\|\mathfrak{H}_{\infty}^{\prime}\right\|_{L^{\infty}\left(\partial \mathcal{D}_{0, \epsilon)}\right.}\right\} .
\end{aligned}
$$

Then, since $\chi_{N, \mathcal{D}}=\chi_{\infty ; \epsilon}+\mathrm{O}\left(\frac{1}{N T}\right)$, it is enough to take $T$ and $N$ large enough so as to get

$$
\left\|0_{T, N}[\xi]\right\|_{L^{\infty}\left(\mathcal{S}_{\zeta \mathrm{m} / 2}\right)} \leq 2\left\|\chi_{\infty ; \epsilon}\right\|_{L^{\infty}\left(\mathcal{S}_{\zeta \mathrm{m} / 2}\right)} .
$$

This entails the claim.

Let $0_{T}$ be defined as

$$
0_{T}[\xi](\lambda)=\chi_{\infty ; \epsilon}(\lambda)+\frac{1}{T} \oint_{\partial \mathcal{D}_{0, \epsilon}} \mathrm{d} u K(\lambda-u) \int_{\kappa}^{u} \mathrm{~d} v \int_{0}^{1} \mathrm{~d} t G\left[\xi-e_{0}\right](v, t)
$$

with

$$
\chi_{\infty ; \epsilon}(\lambda)=-\oint_{\partial \mathcal{D}_{0, \epsilon}} \frac{K(\lambda-u)}{1+\mathrm{e}^{-\mathfrak{H}_{\infty}(u)}} \cdot e_{0}(u) \cdot \mathrm{d} u
$$

and the $v$ integral in (5.38) running along $\partial \mathcal{D}_{0, \epsilon}$. 
Theorem 5.4. There exist $N_{0}, T_{0}$ such that, for any $N>N_{0}$ and $T>T_{0}$ :

- the operator $O_{T, N}$ admits a unique fixed point in $\mathcal{B}_{i}$;

- the fixed point is continuous in $N \geq N_{0}$ and converges, when $N \rightarrow+\infty$, to the unique fixed point of $O_{T}$ in $\mathcal{B}_{\mathrm{c}}$.

The existence of a unique fixed point for $O_{T}$ is part of the conclusions.

Proof -

One starts by showing that $0_{T, N}$ is strictly contractive on $\mathcal{B}_{\mathfrak{c}}$, viz. for any $\xi_{1}, \xi_{2} \in \mathcal{B}_{\mathfrak{c}}$,

$$
\left\|0_{T, N}\left[\xi_{1}\right]-0_{T, N}\left[\xi_{2}\right]\right\|_{L^{\infty}\left(\mathcal{S}_{\zeta \mathrm{m} / 2}\right)} \leq \frac{C}{T} \cdot\left\|\xi_{1}-\xi_{2}\right\|_{L^{\infty}\left(\mathcal{S}_{\zeta \mathrm{m} / 2}\right)}
$$

for some constant $C>0$. Notice that

$$
\gamma_{\xi_{1}}^{2}-\gamma_{\xi_{2}}^{2}=\left(\xi_{1}-\xi_{2}\right)\left(\xi_{1}+\xi_{2}-2 \varpi_{N}\right) .
$$

Likewise, one has

$$
\begin{aligned}
& \gamma_{\xi_{1}}(v) \gamma_{\xi_{1}}^{\prime}(v) \cdot \partial_{2} \mathscr{L}\left(v, \frac{t \gamma_{\xi_{1}}(v)}{T}\right)-\gamma_{\xi_{2}}(v) \gamma_{\xi_{2}}^{\prime}(v) \cdot \partial_{2} \mathscr{L}\left(v, \frac{t \gamma_{\xi_{2}}(v)}{T}\right) \\
& =\frac{1}{2} \partial_{v}\left[\gamma_{\xi_{1}}^{2}(v)-\gamma_{\xi_{2}}^{2}(v)\right] \cdot \partial_{2} \mathscr{L}\left(v, \frac{t \gamma_{\xi_{1}}(v)}{T}\right)+\frac{1}{2} \partial_{v}\left[\gamma_{\xi_{2}}^{2}(v)\right] \cdot\left\{\partial_{2} \mathscr{L}\left(v, \frac{t \gamma_{\xi_{1}}(v)}{T}\right)-\partial_{2} \mathscr{L}\left(v, \frac{t \gamma_{\xi_{2}}(v)}{T}\right)\right\},
\end{aligned}
$$

which allows one to express the difference involving the $\mathscr{L}$ 's by a first order Taylor integral formula

$$
\mathscr{L}(v, x)=\mathscr{L}(v, 0)+x \int_{0}^{1} \partial_{2} \mathscr{L}(v, t x) \mathrm{d} t .
$$

By proceeding analogously relatively to the second term present in (5.21) one obtains

$$
\begin{aligned}
& G\left[\gamma_{\xi_{1}}\right](v, t)-G\left[\gamma_{\xi_{2}}\right](v, t)= \frac{1}{2} \partial_{v}\left\{\left(\xi_{1}-\xi_{2}\right)(v)\left(\xi_{1}+\xi_{2}-2 \varpi_{N}\right)(v)\right\} \cdot \partial_{2} \mathscr{L}\left(v, \frac{t \gamma_{\xi_{1}}(v)}{T}\right) \\
&+\frac{t}{2 T}\left(\xi_{1}-\xi_{2}\right)(v) \partial_{v}\left[\gamma_{\xi_{2}}^{2}(v)\right] \cdot \int_{0}^{1} \partial_{2}^{2} \mathscr{L}\left(v, \frac{t \gamma_{\xi_{2}}(v)}{T}+\frac{t x}{T}\left[\gamma_{\xi_{1}}(v)-\gamma_{\xi_{2}}(v)\right]\right) \mathrm{d} x \\
&+(1-t) \mathfrak{W}_{\infty}^{\prime}(v)\left\{\left(\xi_{1}-\xi_{2}\right)(v)\left(\xi_{1}+\xi_{2}-2 \varpi_{N}\right)(v) \cdot \partial_{2}^{2} \mathscr{L}\left(v, \frac{t \gamma_{\xi_{1}}(v)}{T}\right)\right. \\
&\left.+\frac{t}{T}\left(\xi_{1}-\xi_{2}\right)(v) \gamma_{\xi_{2}}^{2}(v) \cdot \int_{0}^{1} \partial_{2}^{3} \mathscr{L}\left(v, \frac{t \gamma_{\xi_{2}}(v)}{T}+\frac{t x}{T}\left[\gamma_{\xi_{1}}(v)-\gamma_{\xi_{2}}(v)\right]\right) \mathrm{d} x\right\} .
\end{aligned}
$$

This representation readily allows one to infer that there exists a constant $C>0$ such that

$$
\left\|0_{T, N}\left[\xi_{1}\right]-0_{T, N}\left[\xi_{2}\right]\right\|_{L^{\infty}\left(\mathcal{S}_{\zeta \mathrm{m} / 2}\right)} \leq \frac{C}{T}\left\|\xi_{1}-\xi_{2}\right\|_{L^{\infty}\left(\partial \mathcal{D}_{0, \epsilon}\right)}
$$

from where the strict contractivity of $\mathrm{O}_{T, N}$ follows provided that $T$ is large enough. Thus, by the Banach fixed point theorem, $\mathrm{O}_{T, N}$ admits a unique fixed point in $\mathcal{B}_{\mathrm{c}}$.

Furthermore, by dominated convergence and the previous bounds, one has that, for any $\xi \in \mathcal{B}_{\mathfrak{c}}$, the map $N \mapsto 0_{T, N}[\xi]$ is continuous with a uniform in $N$ strict contractivity constant. This ensures that the solution is continuous in $N$ as well. The claims relative to the operator $0_{T}$ follow from this continuity. 


\section{The free energy and correlation lengths at high temperatures}

\subsection{Identification of the dominant Eigenvalue}

Theorem 6.1. There exist $T_{0}>0, N_{0}>0$ such that, uniformly in $N \geq N_{0}$ and $T \geq T_{0}$, the quantum transfer matrix admits a non-degenerate dominant Eigenvalue $\widehat{\Lambda}_{\max }$. There exist $\epsilon>0$ such that $\widehat{\Lambda}_{\max }$ admits the integral representation

$$
\widehat{\Lambda}_{\max }=\left(\frac{\sinh (\boldsymbol{\aleph} / N+\mathrm{i} \zeta)}{\sinh (\mathrm{i} \zeta)}\right)^{2 N} \exp \left\{\frac{h}{2 T}-\oint_{\partial \mathcal{D}_{0, \epsilon}} \frac{\sin (\zeta) \cdot \mathcal{L} \mathrm{n}\left[1+\mathrm{e}^{\widehat{\mathfrak{I}}_{\max }}\right](u)}{\sinh (u-\mathrm{i} \zeta) \sinh (u)} \cdot \frac{\mathrm{d} u}{2 \pi}\right\}
$$

which involves the unique solution to the non-linear integral equation on $\mathcal{B}_{c}$ :

$$
\widehat{\mathfrak{A}}_{\max }(\xi)=-\frac{h}{T}+\mathfrak{w}_{N}(\xi)+\oint_{\partial \mathcal{D}_{0, \epsilon}} K(\xi-u) \cdot \mathcal{L} \mathrm{n}\left[1+\mathrm{e}^{\widehat{\mathfrak{A}}_{\max }}\right](u) \cdot \mathrm{d} u
$$

Proof - Let $\widehat{\xi}_{\text {max }}$ be the unique fixed point of the operator $0_{T, N}$ in (5.22) associated with the choices $s=0, y=\emptyset$ and $\mathfrak{X}=\emptyset$. Since $\mathfrak{A}_{\infty}=0$, and since $\varpi_{N}$ is bounded for $T$ large enough, uniformly in $N$ on $\partial \mathcal{D}_{0, \epsilon}$, it follows that the associated function $\widehat{\mathfrak{A}}_{\text {max }}(5.26)$ solving the non-linear integral equation (4.13) is such that

$$
\widehat{\mathfrak{A}}_{\max }=\mathrm{O}\left(T^{-1}\right) \quad \text { uniformly on } \quad \partial \mathcal{D}_{0, \epsilon} .
$$

Furthermore, it holds that

$$
\exp \left\{\widehat{\mathfrak{A}}_{\max }(\lambda)\right\}=\left(\frac{\lambda-\boldsymbol{\aleph} / N}{\lambda+\boldsymbol{\aleph} / N}\right)^{N} \cdot \mathrm{e}^{\frac{1}{T} \widehat{\mathfrak{A}}_{\mathrm{eff}}(\lambda)}
$$

where, upon defining $\sinh c(\lambda)=\sinh (\lambda) / \lambda$, we have set

$$
\widehat{\mathfrak{A}}_{\mathrm{eff}}(\lambda)=\widehat{\boldsymbol{\xi}}_{\max }(\lambda)+N T \ln \left(\frac{\sinh (\lambda+\boldsymbol{\kappa} / N-\mathrm{i} \zeta)}{\sinh (\lambda-\boldsymbol{\kappa} / N-\mathrm{i} \zeta)}\right)+N T \ln \left(\frac{\operatorname{sinhc}(\lambda-\boldsymbol{\kappa} / N)}{\sinh (\lambda+\boldsymbol{\kappa} / N)}\right)-h .
$$

It is easy to see that $\left\|\widehat{\mathscr{A}}_{\mathrm{eff}}\right\|_{L^{\infty}\left(\mathcal{D}_{0, \epsilon}\right)}<C$ uniformly in $N$ and $T$ large enough. This representation shows explicitly that $\mathrm{e}^{\widehat{\mathfrak{A}}_{\max }}$ admits an $N^{\text {th }}$-order pole at $-\boldsymbol{\aleph} / N$ and no other singularities on $\mathcal{D}_{0, \epsilon}$. Hence, the zero monodromy condition

$$
\oint_{\partial \mathcal{D}_{0, \epsilon}} \frac{\mathrm{d} \lambda}{2 \mathrm{i} \pi} \frac{\widehat{\mathfrak{A}}_{\max }^{\prime}(\lambda)}{1+\mathrm{e}^{-\widehat{\mathscr{I}}_{\max }(\lambda)}}=0
$$

entails that $1+\mathrm{e}^{\widehat{\mathfrak{I}}_{\max }}$ admits $N$ zeroes, counted with multiplicities, inside of $\mathcal{D}_{0, \epsilon}$. Let $z$ be any such zero. The explicit form for $\widehat{\mathfrak{A}}_{\text {eff }}$ given in $(6.5)$, leads, upon taking the $N^{\text {th }}$ root, to

$$
z-\frac{\boldsymbol{\aleph}}{N}=\left(z+\frac{\boldsymbol{\aleph}}{N}\right) \cdot \mathrm{e}^{\mathrm{i} \psi_{N}} \quad \text { with } \quad \psi_{N}=\frac{1}{N}\left\{(2 p-1) \pi+\frac{\mathrm{i}}{T} \widehat{\mathfrak{A}}_{\mathrm{eff}}(z)\right\}
$$

for some $p \in Z_{N}$, where $Z_{N}=\llbracket-N / 2+1 ; N / 2 \rrbracket$ if $N$ is even, $Z_{N}=\llbracket-(N-1) / 2 ;(N-1) / 2 \rrbracket$ if $N$ is odd. One then readily concludes that

$$
z-\frac{\boldsymbol{\aleph}}{N}=\frac{2 \boldsymbol{\aleph} \cdot \mathrm{e}^{\mathrm{i} \psi_{N}}}{N \cdot\left[1-\mathrm{e}^{\mathrm{i} \psi_{N}}\right]} \quad \text { and } \quad z+\frac{\boldsymbol{\aleph}}{N}=\frac{2 \boldsymbol{\aleph}}{N \cdot\left[1-\mathrm{e}^{\mathrm{i} \psi_{N}}\right]}
$$


The root $z$ has multiplicity higher or equal to 2 if and only if

$$
\left(1+\mathrm{e}^{\widehat{\mathfrak{A}}_{\max }}\right)^{\prime}(z)=-\widehat{\mathfrak{A}}_{\max }^{\prime}(z)=0 .
$$

The above allows one to compute $\widehat{\mathfrak{A}}_{\max }^{\prime}(z)$ explicitly in the form

$$
\begin{aligned}
\widehat{\mathfrak{H}}_{\max }^{\prime}(z) & =2 \frac{N^{2}}{\boldsymbol{\aleph}} \cdot \sin ^{2}\left(\frac{\psi_{N}}{2}\right)+\frac{1}{T} \widehat{\mathfrak{H}}_{\mathrm{eff}}^{\prime}(z) \\
& =\frac{\pi^{2}(2 p-1)^{2}}{2 \boldsymbol{N}}\left\{1+\mathrm{i} \frac{\widehat{\mathfrak{A}}_{\mathrm{eff}}(z)}{\pi T(2 p-1)}\right\}^{2} \cdot\left(\frac{\sin \left[\psi_{N} / 2\right]}{\psi_{N} / 2}\right)^{2}+\frac{1}{T} \widehat{\mathfrak{H}}_{\mathrm{eff}}^{\prime}(z) .
\end{aligned}
$$

Pick $1 / 2>c>0$ small enough, then uniformly in $N, T$ large enough and for any $p \in Z_{N}$

$$
\mathfrak{R}\left(\psi_{N}\right) \in[-\pi(1+c) ; \pi(1+c)] \quad \text { and } \quad \mathfrak{J}\left(\psi_{N}\right) \in\left[-\frac{c}{T} ; \frac{c}{T}\right] \quad \text { viz. }\left|\frac{\sin \left[\psi_{N} / 2\right]}{\psi_{N} / 2}\right|>C^{\prime}
$$

for some $N$ and $T$ independent constant $C^{\prime}>0$. This is enough to conclude that $\widehat{\mathfrak{A}}_{\max }^{\prime}(z) \neq 0$ and that all the zeroes of $1+\mathrm{e}^{\widehat{\mathfrak{I}}_{\max }}$ on $\mathcal{D}_{0, \epsilon}$ are necessarily simple. Let $\lambda_{1}^{(\max )}, \ldots, \lambda_{N}^{(\max )}$ denote these distinct zeroes.

By tracing backwards the steps that led to the construction of the non-linear integral equation, viz. taking the non-linear terms by means of residues, one obtains

$$
\mathrm{e}^{\widehat{\mathfrak{I}}_{\max }(\xi)}=\mathrm{e}^{-\frac{h}{T}} \prod_{k=1}^{N}\left\{\frac{\sinh \left(\mathrm{i} \zeta-\xi+\lambda_{k}^{(\max )}\right)}{\sinh \left(\mathrm{i} \zeta+\xi-\lambda_{k}^{(\max )}\right)}\right\} \cdot\left\{\frac{\sinh (\xi-\boldsymbol{\aleph} / N) \sinh (\mathrm{i} \zeta+\xi+\boldsymbol{\aleph} / N)}{\sinh (\xi+\boldsymbol{\kappa} / N) \sinh (\mathrm{i} \zeta-\xi+\boldsymbol{\aleph} / N)}\right\}^{N}
$$

Thus, it exactly coincides with the functional form of the auxiliary function introduced in (4.18). Since the zeroes $\lambda_{1}^{(\max )}, \ldots, \lambda_{N}^{(\max )}$ are pairwise distinct, the vector $\Psi\left(\left\{\lambda_{a}^{(\max )}\right\}_{a=1}^{N}\right)$ introduced in (4.2) does indeed produce an Eigenvector of $t_{\mathrm{q}}$. The associated Eigenvalue (4.6) then has the integral representation

$$
\tau\left(0 \mid\left\{\lambda_{a}\right\}_{a=1}^{M}\right)=\left(\frac{\sinh (\boldsymbol{\aleph} / N+\mathrm{i} \zeta)}{\sinh (\mathrm{i} \zeta)}\right)^{2 N} \exp \left\{\frac{h}{2 T}-\oint_{\partial \mathcal{D}_{0, \epsilon}} \frac{\sin (\zeta) \mathcal{L}\left[1+\mathrm{e}^{\widehat{\mathscr{A}}_{\max }}\right](u)}{\sinh (u-\mathrm{i} \zeta) \sinh (u)} \cdot \frac{\mathrm{d} u}{2 \pi}\right\} .
$$

Since the result does not depend on the choice of $\kappa$, one may as well set $\kappa=\epsilon$, which allows one to get rid of the last term. It is then readily seen that the integral term appearing in the Eigenvalue of $t_{q}$ associated with this solution exhibits the large- $T$ behaviour

$$
\oint_{\partial \mathcal{D}_{0, \epsilon}} \frac{\sin (\zeta) \mathcal{L} \mathrm{n}\left[1+\mathrm{e}^{\widehat{\mathfrak{A}}_{\max }}\right](u)}{\sinh (u-\mathrm{i} \zeta) \sinh (u)} \cdot \frac{\mathrm{d} u}{2 \pi}=-\ln 2+\mathrm{O}\left(T^{-1}\right)
$$

Thence, (6.14) entails that the associated Eigenvalue of the quantum transfer matrix takes the form

$$
\tau\left(0 \mid\left\{\lambda_{a}^{(\max )}\right\}_{a=1}^{N}\right)=2+\mathrm{O}\left(T^{-1}\right) .
$$

Thus, by virtue of Proposition 3.1, $\tau\left(0 \mid\left\{\lambda_{a}^{(\max )}\right\}_{a=1}^{N}\right)$ does coincide with the dominant Eigenvalue $\widehat{\Lambda}_{\max }$.

All is now set in place so as to allow for the proof of Theorem 1.1 stated in the introduction

Proof- 
It is a corollary of Proposition 3.1, Theorem 3.2, the classical result for the existence of the limit (1.4) and the one for the limit (3.15) that

$$
-\frac{f}{T}=\lim _{N \rightarrow+\infty} \ln \widehat{\Lambda}_{\max } .
$$

The existence of the Trotter limit of the solution $\widehat{\mathfrak{A}}_{\max }$ to the non-linear integral equation, and the convergence, uniformly on $\partial \mathcal{D}_{0, \epsilon}$ of $\widehat{\mathfrak{A}}_{\max }$ to the solution $\mathfrak{H}_{\max }$ adjoined to the integral representation for $\widehat{\Lambda}_{\text {max }}$ provided in Theorem 6.1 then allows one to conclude.

\subsection{Sub-dominant Eigenvalues at high temperatures}

In this section we study the solvability of the non-linear integral equation problem associated with the infinite Trotter number limit, namely the existence of solutions $\mathfrak{A}$ to the non-linear integral equation (4.32) subject to additional constraints (4.36) on the monodromy of the solution and on the parameters building up the particlehole sets. We focus only on the characterisation of a subset of solutions, namely those whose sets $\mathfrak{X}$ and $\mathcal{Y}$ belong to the class $C_{\alpha, \varrho}^{\epsilon}$ with fixed cardinalities $n_{x}, n_{y}$ and parameters $\epsilon, \alpha, \varrho$ small enough but finite. While it is not a problem to carry out the same analysis at finite Trotter number, such an analysis would be of limited use in that, from the point of view of physical applications, one is interested, in the end, in results at infinite Trotter number.

Recall that, by virtue of Proposition 5.3 and Theorem 5.4 given $\alpha, \varrho>0, n_{x}, n_{y} \in \mathbb{N}$ fixed, there exist $T_{0}>0$ and $\epsilon>0$ such that any solution to the non-linear integral equation (4.32) with $\mathfrak{X}, \mathcal{Y}$ belonging to the class $C_{\alpha, \varrho}^{\epsilon}$ with cardinalities $n_{x}, n_{y}$ takes the form

$$
\mathfrak{A}=\mathfrak{A}_{\infty}+\frac{1}{T} \mathfrak{b} \quad \text { with } \quad \mathfrak{b}=\xi-e_{0} .
$$

Here $\xi$ is the unique fixed point of the operator $0_{T}(5.38)$ associated with the roots $\mathfrak{X}, \mathcal{Y}$ and the function $\mathfrak{b}$ is bounded on $\partial \mathcal{D}_{0, \epsilon}$. Below we study the solvability, in the large- $T$ regime, of the additional constraints (4.36) assuming that $\mathfrak{X}, \mathcal{Y}$ belong to the class $C_{\alpha, \varrho}^{\epsilon}$ with cardinalities $n_{x}, n_{y}$.

Let $\mathcal{Y}=\left\{y_{1}, \ldots, y_{n_{y}}\right\}$ and $\mathcal{Y}^{\prime}=\left\{y_{1}^{\prime}, \ldots, y_{n_{y}}^{\prime}\right\}$ be two sets of equal cardinality. One defines the distance between the sets $\mathcal{Y}$ and $\mathcal{Y}^{\prime}$ as

$$
\mathrm{d}\left(\boldsymbol{Y}, \boldsymbol{y}^{\prime}\right)=\min _{\sigma \in \subseteq_{n_{y}}}\left\{\sum_{a=1}^{n_{y}}\left|y_{a}-y_{\sigma(a)}^{\prime}\right|\right\} .
$$

Further, define the collection of solution sets to the Bethe Ansatz equations connected with the ordinary transfer matrix of the spin-1 XXZ chain:

$\sigma_{\infty}=\left\{\boldsymbol{y}_{\infty}=\left\{y_{\infty ; 1}, \ldots, y_{\infty ; n_{y}}\right\}: \forall a \in \llbracket 1 ; n_{y} \rrbracket, \quad(-1)^{s+1} \lim _{u \rightarrow y_{\infty ; a}} \prod_{b=1}^{n_{y}}\left\{\frac{\sinh \left(\mathrm{i} \zeta+y_{\infty ; b}-u\right)}{\sinh \left(\mathrm{i} \zeta+u-y_{\infty ; b}\right)}\right\} \cdot\left(\frac{\sinh (\mathrm{i} \zeta+u)}{\sinh (\mathrm{i} \zeta-u)}\right)^{n_{x}}=1\right\}$

Note that this way of defining the solution set allows, in principle, for roots located at $\infty$. Indeed, the limit of some roots going to $\infty$ is well-defined within the prescription used for defining the full solution set $\sigma_{\infty}$.

One defines the distance of a set $\mathcal{Y}$ of cardinality $n_{y}$ to $\sigma_{\infty}$ as

$$
\mathrm{d}\left(\sigma_{\infty}, \boldsymbol{Y}\right)=\min _{\boldsymbol{y}_{\infty} \in \sigma_{\infty}}\left\{\mathrm{d}\left(\boldsymbol{Y}_{\infty}, \boldsymbol{Y}\right)\right\}
$$


Proposition 6.2. Let $\alpha, \varrho>0, n_{x}, n_{y} \in \mathbb{N}$ be fixed. Let $\mathfrak{x}$ and $\mathcal{Y}$ belong to the class $\mathcal{C}_{\alpha, \varrho}^{\epsilon}$ with cardinalities $n_{x}, n_{y}$ and let $T_{0}>0$ and $\epsilon>0$ be such that the non-linear integral equation (4.32) admits a unique solution.

If $\mathfrak{X}=\left\{x_{1}, \ldots, x_{n_{x}}\right\}$ and $\mathcal{Y}=\left\{y_{1}, \ldots, y_{n_{y}}\right\}$ are $T$-dependent sets satisfying the subsidiary conditions (4.36) uniformly in $T$ large enough, then necessarily

- $|\mathfrak{X}|=|\mathcal{Y}|+s$;

- there exist integers $k_{a} \in \mathbb{Z}$ such that the "hole roots" take the form

$$
x_{a}=\frac{-2 J \sin (\zeta)}{T\left[\left(2 k_{a}+1+s\right) \pi-\sum_{y \in \mathcal{Y}} \theta(-y)\right]}+\mathrm{O}\left(T^{-2}\right) \quad a=1, \ldots, n_{x} ;
$$

- $\mathrm{d}\left(\sigma_{\infty}, \mathcal{Y}\right)=\mathrm{o}(1)$, where the control on the remainder only depends on $\epsilon, \varrho, \alpha, n_{x}$ and $n_{y}$.

Note that the integers $k_{a}$ appearing in the statement of the proposition may, in principle, dependent on $T$.

Proof-

The expansion (6.18) ensures that

$$
\frac{\mathfrak{H}^{\prime}(\lambda)}{1+\mathrm{e}^{-\mathfrak{H}(\lambda)}}=\frac{\mathfrak{A}_{\infty}^{\prime}(\lambda)}{1+\mathrm{e}^{-\mathfrak{H}_{\infty}(\lambda)}}+\frac{1}{T} \partial_{\lambda}\left\{\frac{\mathrm{b}(\lambda)}{1+\mathrm{e}^{-\mathfrak{H}_{\infty}(\lambda)}}\right\}+\mathrm{O}\left(\frac{1}{T^{2}}\right),
$$

with the $\mathrm{O}\left(T^{-2}\right)$ remainder being uniform on $\partial \mathcal{D}_{0, \epsilon}$.

Upon inserting the above expansion into the monodromy constraint (4.36), one obtains that

$$
\oint_{\partial \mathcal{D}_{0, \epsilon}} \frac{\mathfrak{H}_{\infty}^{\prime}(\xi)}{1+\mathrm{e}^{-\mathfrak{H}_{\infty}(\xi)}} \cdot \frac{\mathrm{d} u}{2 \mathrm{i} \pi}+\mathrm{O}\left(\frac{1}{T}\right)=|\mathfrak{X}|-|\boldsymbol{y}|-s,
$$

since the sets $\mathfrak{X}, \mathcal{Y}$, belonging to the class $C_{\alpha, \varrho}^{\epsilon}$ with $\epsilon>0$ small enough, do not give rise to singular roots. However, due to (5.13), the first integral vanishes. Since the lhs is integer valued, and since the sole dependence on $\mathfrak{X}, \mathcal{Y}$ of the remainder $\mathrm{O}\left(T^{-1}\right)$ is bounded by $|\mathfrak{X}|+|\mathcal{Y}|$, one concludes that, for any $T>T_{0}^{\prime}$ for some $T_{0}^{\prime}$ large enough, in case of sets $\mathfrak{X}, \mathcal{Y}$ belonging to the class $C_{\alpha, \varrho}^{\epsilon}$, one may produce solutions to the joint problem (4.36) if and only if the sets' cardinalities are constrained as

$$
|\mathfrak{X}|=|\mathcal{Y}|+s .
$$
(4.36).

We now analyse more precisely the structure of the sets $\mathfrak{X}$ and $\mathcal{Y}$ satisfying to the subsidiary constraints in

\section{- The hole set $\mathfrak{X}$}

One starts by determining elements forming the hole set $\mathfrak{X}$. By virtue of 6.18$), b$ is meromorphic on $\mathcal{D}_{0, \epsilon}$ with a single pole at $\xi=0$ and such that

$$
\operatorname{Res}(\mathfrak{b}(\xi) \mathrm{d} \xi, \xi=0)=-2 \mathrm{i} J \sin (\zeta) .
$$

As a consequence, there exits a holomorphic function $\mathfrak{b}^{(r)}$ on $\mathcal{D}_{0, \epsilon}$ that is bounded uniformly in $T$ large enough and such that

$$
\mathfrak{b}(\xi)=-\frac{2 \mathrm{i} J}{\xi} \sin (\zeta)+\mathfrak{b}^{(r)}(\xi) .
$$


Any hole root $x$ will satisfy the equation

$$
\mathfrak{A}_{\infty}(x)+\frac{\mathfrak{b}(x)}{T}=\left(2 k_{x}+1\right) \mathrm{i} \pi \quad \text { for some } k_{x} \in \mathbb{Z} .
$$

By virtue of (5.13), it holds that $\mathrm{d}\left(\mathfrak{H}_{\infty}(x), \mathrm{i} \pi+2 \mathrm{i} \pi \mathbb{Z}\right)>C_{\varrho}$ for some $C_{\varrho}>0$ depending on the parameter $\varrho>0$. Hence, any solution to (6.28) has to be such that $|\mathfrak{b}(x) / T|>C_{\varrho}$. The form of the behaviour around the origin (6.27) then entails that $T x$ has to be uniformly bounded. Thus, one reparametrises $x=u_{x} / T$, with $u_{x}$ bounded in $T$, which leads to the equation

$$
\left(2 k_{x}+1\right) \mathrm{i} \pi=\mathfrak{A}_{\infty}(0)-\frac{2 \mathrm{i} J}{u_{x}} \sin (\zeta)+\mathrm{O}\left(T^{-1}\right) .
$$

Thus

$$
u_{x}=\frac{-2 \mathrm{i} J \sin (\zeta)}{\left(2 k_{x}+1\right) \mathrm{i} \pi-\mathfrak{A}_{\infty}(0)}+\mathrm{O}\left(T^{-1}\right)
$$

This entails that all hole roots converge to 0 with speed at least $\mathrm{O}\left(T^{-1}\right)$. Note that this convergence may be faster than $T^{-1}$ in case of integers $k_{x}$ also going to infinity with $T$.

\section{- The particle set $y$}

The very definition of the class $C_{\alpha, \varrho}^{\epsilon}$ entails that particle roots $y \in \mathcal{Y}$ are such that $y \pm \mathrm{i} \zeta \notin \mathcal{D}_{0, \epsilon}$. Then, one has

$$
\mathrm{e}^{\mathfrak{H}(\lambda)}=\mathrm{e}^{\mathfrak{H}_{\infty}(\lambda)} \cdot \mathrm{e}^{\frac{\mathrm{b}(\lambda)}{T}} \quad \text { for } \quad \lambda \in \mathcal{S}_{\frac{\pi}{2}} \backslash\left\{\mathcal{D}_{0, \epsilon} \cup_{v= \pm} \mathcal{D}_{v i \zeta_{\mathrm{m}}, \alpha}\right\}
$$

and thus any root $y \in \mathcal{Y}$ satisfies

$$
(-1)^{s+1} \cdot \lim _{u \rightarrow y}\left\{\prod_{y^{\prime} \in \mathcal{Y} \ominus \mathfrak{X}} \frac{\sinh \left(\mathrm{i} \zeta+y^{\prime}-u\right)}{\sinh \left(\mathrm{i} \zeta+u-y^{\prime}\right)}\right\} \cdot \mathrm{e}^{\mathrm{O}\left(T^{-1}\right)}=1 .
$$

Note that the limit procedure provides one with a prescription for treating roots at $\infty$ and also for regularising potential zeroes and poles which cancel eventually out between the numerator and the denominator. It follows from the above that $x=\mathrm{O}\left(T^{-1}\right)$ for any $x \in \mathfrak{X}$. Thus, the properties of the class $C_{\alpha, \varrho}^{\epsilon}$ allow one to recast the above equation in the form

$$
(-1)^{s+1} \cdot \lim _{u \rightarrow y}\left\{\prod_{y^{\prime} \in \mathcal{Y}} \frac{\sinh \left(\mathrm{i} \zeta+y^{\prime}-u\right)}{\sinh \left(\mathrm{i} \zeta+u-y^{\prime}\right)}\right\} \cdot\left\{\frac{\sinh (\mathrm{i} \zeta+y)}{\sinh (\mathrm{i} \zeta-y)}\right\}^{n_{x}} \cdot \mathrm{e}^{\mathrm{O}\left(T^{-1}\right)}=1 .
$$

Now assume that $\mathcal{Y}$ is a one parameter $T$ family of particle sets solving the constraints (4.36) and such that $\mathrm{d}\left(\mathcal{Y}, \sigma_{\infty}\right) \nrightarrow 0$ as $T \rightarrow \infty$. Thus, one may extract a sequence of sets $\mathcal{Y}_{n}$ associated with a sequence of temperatures $T_{n} \rightarrow+\infty$ such that $\mathrm{d}\left(\mathcal{Y}_{n}, \sigma_{\infty}\right)>\gamma$ for some $\gamma>0$. By taking the $n \rightarrow+\infty$ on the level of the associated equation (6.33), one gets that the limiting set $\mathcal{Y}_{\infty} \in \sigma_{\infty}$, which is a contradiction to $\mathrm{d}\left(\mathcal{Y}_{n}, \sigma_{\infty}\right)>\gamma$. This entails the claim.

Basically, Proposition 6.2 states that, for temperatures large enough, for the solutions to the non-linear integral equations describing the Eigenvalues of the quantum transfer matrix with the hole and particle sets $\mathfrak{X}, \mathcal{Y}$ belonging to a class $C_{\alpha, \varrho}^{\epsilon}$ with cardinalities $n_{x}, n_{y}$, any element of the hole set collapses to 0 with speed $T^{-1}$, and the leading 
behaviour of this collapse is parametrised by a collection of integers. In their turn, the elements of the particle set $\mathcal{Y}$ essentially collapse onto the zero set, on $\mathcal{S}_{\frac{\pi}{2}} \backslash\left\{\mathcal{D}_{0, \epsilon} \cup_{v= \pm} \mathcal{D}_{\mathrm{i} v \zeta_{\mathrm{m}}, \alpha}\right\}$, of the map

$$
f:\left\{\begin{array}{ccc}
\mathbb{C}^{|\mathcal{Y}|} & \rightarrow \mathbb{C}^{|\mathcal{Y}|} \\
y & \mapsto f(y)
\end{array} \quad \boldsymbol{f}(\boldsymbol{y})=\left(f_{1}(y), \cdots, f_{|y|}(y)\right),\right.
$$

with

$$
\boldsymbol{f}_{b}(\boldsymbol{y})=1+(-1)^{s} \cdot \prod_{a=1}^{|\underline{y}|} \frac{\sinh \left(\mathrm{i} \zeta+y_{a}-y_{b}\right)}{\sinh \left(\mathrm{i} \zeta+y_{b}-y_{a}\right)} \cdot\left(\frac{\sinh \left(\mathrm{i} \zeta+y_{b}\right)}{\sinh \left(\mathrm{i} \zeta-y_{b}\right)}\right)^{|\mathcal{Y}|+s} .
$$

So as to be more precise relatively to the speed of the convergence of the set $\mathcal{Y}$, one would need to have more information on the behaviour of $\boldsymbol{f}$ in the vicinity of its zeroes. In particular, if $\boldsymbol{y}_{\infty}=\left(y_{\infty ; 1}, \cdots, y_{\infty ;|\boldsymbol{y}|}\right)$ is a zero of $\boldsymbol{f}$ and the differential of $\boldsymbol{f}$ at $\boldsymbol{y}_{\infty}$ is invertible, then if $\boldsymbol{y}=\left(y_{1}, \ldots, y_{|y|}\right)$ is the vector built up from the elements of the particle set $\boldsymbol{Y}$, and if it holds that $\boldsymbol{y}=\boldsymbol{y}_{\infty}+\mathrm{o}(1)$, where the remainder is to be understood coordinate-wise when $T \rightarrow+\infty$, then it is easy to show using the implicit function theorem, that, in fact,

$$
y_{a}=y_{\infty ;|y|}+\mathrm{O}\left(T^{-1}\right) \text {. }
$$

Thus, our analysis shows that, in the high-temperature regime, at least part of the subdominant Eigenvalues of the quantum transfer matrix, and hence the associated correlation lengths, are parameterised by solutions to the Bethe Ansatz equations of the spin-1 XXZ spin chain.

\section{Numerical illustration}

In this section, we illustrate the conclusions of the analysis carried out in the previous Sections 5, 6 by providing a numerical analysis of the solutions to the Bethe Ansatz equations for finite Trotter numbers. The fact that the results are obtained at finite and relatively small Trotter numbers is not a problem when $T$ is high enough, in that the convergence of the infinite Trotter number limit results is controlled as $\mathrm{O}\left([T N]^{-1}\right)$. Our analysis deals with the Trotter numbers $N=4,5,6$, and we employed an algorithm proposed in [1]. The latter combines a numerical diagonalisation of the quantum transfer matrix and the use of an expansion of Baxter's TQ relation. The algorithm enables us, for generic $\zeta$, to locate the Bethe roots associated with any Eigenstate and then to find the zeros of the Eigenvalues of the quantum transfer matrix associated with the given Eigenstate easily. This information provides the data for $\widehat{\mathfrak{X}}$ and $\widehat{\mathcal{y}}$.

\subsection{Examples of of Bethe and hole roots}

We first present a few examples of the Bethe roots and hole roots $\widehat{\mathfrak{X}}$.

For the first example, we have determined the solutions to (4.3) with the choice of parameters $N=5, M=$ $5, T=100, \zeta=\frac{\pi}{7}$. Here, for the sake of brevity, we only list three solutions. The Eigenvalues $\widehat{\Lambda}_{a}$, associated Bethe roots and set $\widehat{\mathfrak{X}}$ for the 2nd, the 12-th and 83-th subdominant Eigenvalues of $t_{\mathfrak{q}}(0)$ are gathered in Table 1 , Note that the Eigenvalues are labelled in respect to the non-increasing ordetf of $\left|\widehat{\Lambda}_{a}\right|$.

Among these three states, the 12-th state gives rise to sets $\widehat{\mathfrak{x}}$ and $\widehat{y}$ belonging to a class $C_{\alpha, \varrho}^{\epsilon}$ : the condition (5.1) is violated for any $\varrho>0$ for the 2 nd excited state, while the 83-th excited state contains singular roots 6 , viz. $\widehat{\mathcal{Y}}_{\mathrm{sg}} \neq \emptyset$.

\footnotetext{
${ }^{\dagger}$ In principle, degeneracies of $\left|\widehat{\Lambda}_{a}\right|$ may occur and then the choice of the ordering for the various Eigenvalues with fixed modulus is taken in the direction of increasing arguments, with $\arg \in[-\pi ; \pi[$.

${ }^{5}$ The class $C_{\alpha, \varrho}^{\epsilon}$ is defined in Definition 5.1

${ }^{6}$ To be precise, they are slightly away from $\pm \mathrm{i}$; they are at $\pm 1.00000000629 \cdots \mathrm{i}$
} 


\begin{tabular}{|c|c|c|c|}
\hline & $\widehat{\Lambda}_{a}$ & $\left\{\frac{\lambda_{a}}{\zeta}\right\}$ & $\left\{\frac{\widehat{x}_{a}}{\zeta}\right\}$ \\
\hline 2nd & $-9.19523 \times 10^{-3}$ & $\frac{\left\{\frac{\pi}{2 \zeta} \mathrm{i}, \pm 1.329782 \times 10^{-3},\right.}{\left. \pm 3.14044 \times 10^{-4}\right\}}$ & $\left\{ \pm 6.4790 \times 10^{-2}\right\}$ \\
\hline 12-th & $\begin{array}{c}-4.07262 \times 10^{-4}+ \\
9.0811 \times 10^{-5} \mathrm{i}\end{array}$ & $\begin{array}{c}\left\{2.18463 \times 10^{-7}, 7.02268 \times 10^{-4},\right. \\
2.98366 \times 10^{-3},\end{array}$ & $\begin{array}{c}\left\{-2.97906 \times 10^{-3},\right. \\
\left.7.02935 \times 10^{-4}\right\}\end{array}$ \\
\hline 83-th & $2.01835 \times 10^{-6}$ & $\underline{\left.-1.14080 \times 10^{-3} \pm 0.575835 \mathrm{i}\right\}}$ & $\left.\pm 3.140243 \times 10^{-4}, \pm \mathrm{i}\right\}$ \\
\hline
\end{tabular}

Table 1: Eigenvalues $\widehat{\Lambda}_{a}$, associated Bethe roots and sets $\widehat{\mathfrak{X}}$ and $\widehat{\mathcal{Y}}$ for $N=5, M=5, T=100, \zeta=\frac{\pi}{7}$. The numbers are given with a 6 digit accuracy. The underlined roots are the members of the set $\widehat{y}$.

The examples for the same parameters but with $M=4$ are given in Table 2 .

\begin{tabular}{|l|c|c|c|}
\hline & $\widehat{\Lambda}_{a}$ & $\left\{\frac{\lambda_{a}}{\zeta}\right\}$ & $\left\{\frac{\widehat{x}_{a}}{\zeta}\right\}$ \\
\hline 10 -th & $2.10835 \times 10^{-4}$ & $\left\{\frac{\pi}{2 \zeta} \mathrm{i}, 0, \pm 7.019646 \times 10^{-4}\right\}$ & $\left\{ \pm 2.96294 \times 10^{-3}\right\}$ \\
\hline 17 -th & $1.99318 \times 10^{-4}$ & $\left\{\underline{ \pm 7.15137 \times 10^{-2}}, \pm 3.14057 \times 10^{-4}\right\}$ & $\left\{ \pm 1.32993 \times 10^{-3}\right\}$ \\
\hline 123 -th & $-4.54205 \times 10^{-8}$ & $\left.\underline{\underline{ \pm i}}, \pm 1.88769 \times 10^{-8}\right\}$ & $\begin{array}{l} \pm 2.96082 \times 10^{-3} \\
\left. \pm 7.01891 \times 10^{-4}\right\}\end{array}$ \\
\hline
\end{tabular}

Table 2: Examples of eigenstates: $N=5, M=4, T=100, \zeta=\frac{\pi}{7}$.

In this sector, the 10-th excited state belongs to $C_{\alpha, \varrho}^{\epsilon}, \varrho=0$ for the 17-th excited state and the 123-th excited state contains $\widehat{\mathcal{y}}_{\text {sg }}$.

\subsection{Optimal choice of parameters $\epsilon, \alpha$ and $\varrho$}

The $C_{\alpha, \varrho}^{\epsilon}$ classes provide a convenient subset in the parameter space of particle roots which allows one to easily describe any particle parameters, subject to the constraints (4.11). Ideally, the parameters $\epsilon, \alpha$ and $\varrho$ should be taken as small as possible. However, the analysis developed in Section 6 is not refined enough so as to be able to handle rigorously the presumably most optimal case when these parameters go to zero with some power of $\mathrm{T}$. Also, it is clear that there might be solution sets $\widehat{\mathfrak{X}}, \widehat{y}$ to the constraints 4.11) such that the parameters $\widehat{y}$ do not belong to any class $C_{\alpha, \varrho}^{\epsilon}$. We have investigated these cases numerically.

We set

$$
\delta_{a}:=(-1)^{n_{x}-n_{y}+1} \prod_{b=1}^{n_{y}}\left\{\frac{\sinh \left(\mathrm{i} \zeta+y_{b}-y_{a}\right)}{\sinh \left(\mathrm{i} \zeta+y_{a}-y_{b}\right)}\right\} \cdot\left(\frac{\sinh \left(\mathrm{i} \zeta+y_{a}\right)}{\sinh \left(\mathrm{i} \zeta-y_{a}\right)}\right)^{n_{x}}-1
$$

and regard $\left\{y_{a}\right\}$ as a set of solutions if $\max _{a}\left|\delta_{a}\right|<\delta$ for a fixed small $\delta$.

With this convention we classify all Eigenstates into five cases: 
1. The states with $\mathcal{Y}=\emptyset$.

2. The states containing singular roots $\widehat{\mathcal{y}}_{\mathrm{sg}}$.

3. The states which satisfy

$$
\left|(-1)^{s} \prod_{y \in \widehat{Y}} \frac{\sinh (\mathrm{i} \zeta+y)}{\sinh (\mathrm{i} \zeta-y)}+1\right|<\varrho .
$$

4. The states which belong to $C_{\alpha, \varrho}^{\epsilon}$ and satisfy (7.1) with a fixed $\delta$.

5. The states which belong to $\mathcal{C}_{\alpha, \varrho}^{\epsilon}$ but do not satisfy (7.1) with a fixed $\delta$.

The first three cases do not belong to $C_{\alpha, \varrho}^{\epsilon}$.

The classification depends on the actual choice of parameters $\epsilon, \alpha, \varrho$ and $\delta$. We performed the numerical estimation for various $N, M$ and $T$ and found that a consistent choice is

$$
\epsilon \propto \frac{1}{\sqrt{T}}, \quad \varrho \propto \frac{1}{\sqrt{T}}, \quad \delta \propto \frac{1}{T} .
$$

They are thus expected to be infinitesimally small in the high-temperature limit. On the other hand, the choice of $\alpha$ seems almost independent of $T$ : it does not change the classification for $10^{-4} \leq \alpha \leq 10^{-2}$ for $100 \leq T \leq 1000$.

A rigorous justification of these observations is left as a future problem.

\subsection{Conclusion from numerics}

Taken the above choice for granted, we have verified that each state is classified into either (A) not a member of $C_{\alpha, \varrho}^{\epsilon}$ or (B) a member of $C_{\alpha, \varrho}^{\epsilon}$ and satisfying (7.1) with $\delta$. We tabulate the number of states in each case for $N=6$, $M=5,6$ and $\zeta=\pi / 7$ in Table 3 .

\begin{tabular}{|l|r|r|r|r|}
\hline & case 1 & case 2 & case 3 & case 4 \\
\hline$N=6, M=5, T=100$ & 11 & 26 & 178 & 577 \\
$N=6, M=5, T=1000$ & 11 & 26 & 178 & 577 \\
$N=6, M=6, T=100$ & 1 & 53 & 211 & 659 \\
$N=6, M=6, T=1000$ & 1 & 75 & 189 & 659 \\
\hline
\end{tabular}

Table 3: The distribution of numbers of states into different cases. Here we set $\alpha=0.01, \epsilon=\varrho=0.6 / \sqrt{T}, \delta=$ $10 / T$.

One notices that the majority of states belongs to case 4, namely to class $C_{\alpha, \varrho}^{\epsilon}$, which satisfies the higher-level Bethe ansatz. The distribution of numbers of states in each case seems stable against a change in temperature although it depends on the sector $M$. It also depends on the Trotter number $N$. Although we have tried it only for $N=4,5,6$, we find that the relative number of members in class $C_{\alpha, \varrho}^{\epsilon}$ increases with $N$. The percentage of $C_{\alpha, \varrho}^{\epsilon}$ in the total number of eigenstates for each $N, M$ is given in Table 4, It suggests that most of the states will belong to class $C_{\alpha, \varrho}^{\epsilon}$ in the Trotter limit $N \rightarrow+\infty$. 


\begin{tabular}{|l|c|c|c|}
\hline & $N=4$ & $N=5$ & $N=6$ \\
\hline$M=N$ & 0.586 & 0.655 & 0.713 \\
$M=N-1$ & 0.589 & 0.671 & 0.729 \\
\hline
\end{tabular}

Table 4: The percentage of $C_{\alpha, \varrho}^{\epsilon}$ for several $N, M$.

\subsection{Higher level Bethe Ansatz equation}

Alternatively we can solve the higher level Bethe Ansatz equation directly for the members in $C_{\alpha, \varrho}^{\epsilon}$.

Recall that the higher level Bethe Ansatz equations governing the positions of the particle roots take the form

$$
(-1)^{n_{x}-n_{y}+1} \prod_{b=1}^{n_{y}}\left\{\frac{\sinh \left(i \zeta+y_{b}-y_{a}\right)}{\sinh \left(i \zeta+y_{a}-y_{b}\right)}\right\} \cdot\left(\frac{\sinh \left(i \zeta+y_{a}\right)}{\sinh \left(i \zeta-y_{a}\right)}\right)^{n_{x}}=1 .
$$

However, these are obtained upon simplifying the original form of the equations in the case where the hole roots are small. In the case where the $x_{a}$ s are not sufficiently small in magnitude, one should rather consider

$$
(-1)^{n_{x}-n_{y}+1} \prod_{b=1}^{n_{y}}\left\{\frac{\sinh \left(i \zeta+y_{b}-y_{a}\right)}{\sinh \left(i \zeta+y_{a}-y_{b}\right)}\right\} \cdot \prod_{\ell=1}^{n_{x}} \frac{\sinh \left(i \zeta+y_{a}-x_{\ell}\right)}{\sinh \left(i \zeta-y_{a}+x_{\ell}\right)}=1 .
$$

While (7.2) can be solved directly for the $y_{a}$ s, In order to solve (7.3) w.r.t. $\left\{y_{a}\right\}$, one needs the input data $\left\{x_{\ell}\right\}$. The hole roots are substituted from the values obtained by the algorithm proposed in [1].

First we choose the same parameters with Table 1 and present some examples including the 12-th excited state

\begin{tabular}{|c|c|c|c|c|}
\hline & $\left\{y_{a} / \zeta\right\}$ & $\left\{\widehat{x}_{a} / \zeta\right\}$ & $\left\{y_{a} / \zeta\right\}$ from $(7.2)$ & $\left\{y_{a} / \zeta\right\}$ from $(7.3)$ \\
\hline 12-th & $\begin{array}{c}\left\{-1.140806 \times 10^{-3}\right. \\
\pm 0.575835 \mathrm{i}\}\end{array}$ & $\begin{array}{c}\left\{-2.979061 \times 10^{-3},\right. \\
\left.7.029353 \times 10^{-4}\right\}\end{array}$ & $\{ \pm 0.577223 \mathrm{i}\}$ & $\begin{array}{c}\left\{-1.138063 \times 10^{-3}\right. \\
\pm 0.577224 \mathrm{i}\}\end{array}$ \\
\hline 27-th & $\left\{ \pm 1.379263+\frac{\pi}{2 \zeta} \mathrm{i}\right\}$ & $\left\{ \pm 2.962552 \times 10^{-3}\right\}$ & $\left\{ \pm 1.378826+\frac{\pi}{2 \zeta} \mathrm{i}\right\}$ & $\left\{ \pm 1.378828+\frac{\pi}{2 \zeta} \mathrm{i}\right\}$ \\
\hline 41-th & $\begin{array}{c}-0.381523 \pm \\
0.547636 \mathrm{i} \\
\left.0.764017+\frac{\pi}{2 \tau} \mathrm{i}\right\}\end{array}$ & $\begin{array}{c}\left\{-4.518492 \times 10^{-3}\right. \\
5.582956 \times 10^{-4} \\
\left.2.169682 \times 10^{-3}\right\}\end{array}$ & $\begin{array}{c}\{-0.381245 \pm \\
0.548153 \mathrm{i} \\
\left.0.762489+\frac{\pi}{2 \tau} \mathrm{i}\right\}\end{array}$ & $\begin{array}{c}-0.381847 \pm \\
0.548154 \mathrm{i} \\
\left.0.761903+\frac{\pi}{2 \zeta} \mathrm{i}\right\}\end{array}$ \\
\hline 120 -th & $\begin{array}{c}-0.295790 \pm \\
0.550527 \mathrm{i} \\
0.2961415 \pm \\
0.550527 \mathrm{i}\}\end{array}$ & $\begin{array}{c}\left\{-2.977582 \times 10^{-3},\right. \\
1.183573 \times 10^{-8}, \\
7.024496 \times 10^{-4}, \\
\left.2.977334 \times 10^{-3}\right\}\end{array}$ & $\begin{array}{c}\{ \pm 0.295564 \pm \\
0.551187 \mathrm{i}\}\end{array}$ & $\begin{array}{c}-0.295393 \pm \\
0.551188 \mathrm{i} \\
0.295745 \pm \\
0.551188 \mathrm{i}\}\end{array}$ \\
\hline
\end{tabular}
in Table 5

Table 5: The comparison of the result by the algorithm of [1], and the solutions to higher level Bethe ansatz equations (7.2) and (7.3) : $N=5, M=5, T=100, \zeta=\frac{\pi}{7}$. States are selected "randomly".

Second we choose the same parameters with Table 2 and present examples including the 10-th excited state in Table 6 


\begin{tabular}{|c|c|c|c|c|}
\hline & $\left\{y_{a} / \zeta\right\}$ & $\left\{\widehat{x}_{a} / \zeta\right\}$ & $\left\{y_{a} / \zeta\right\}$ from $(7.2)$ & $\left\{y_{a} / \zeta\right\}$ from $(7.3)$ \\
\hline 10-th & $\left\{\frac{\pi}{2 \zeta} \mathrm{i}\right\}$ & $\left\{ \pm 2.962940 \times 10^{-3}\right\}$ & $\left\{\frac{\pi}{2 \zeta} \mathrm{i}\right\}$ & $\left\{\frac{\pi}{2 \zeta} \mathrm{i}\right\}$ \\
\hline \multirow{2}{*}{24 -th } & $\left\{3.496022 \times 10^{-4}+\right.$ & $\left\{7.021791 \times 10^{-4}\right.$, & $\left\{\frac{\pi}{2 \zeta} \mathrm{i}\right\}$ & $\left\{3.511598 \times 10^{-4}\right.$ \\
& $\left.\frac{\pi}{2 \zeta} \mathrm{i}\right\}$ & $\left.1.404799 \times 10^{-7}\right\}$ & $\left.+\frac{\pi}{2 \zeta} \mathrm{i}\right\}$ \\
\hline \multirow{3}{*}{32 -th } & $\{-0.466727 \pm$ & $\left\{-4.520602 \times 10^{-3}\right.$, & $\{-0.465800 \pm$ & $\{-0.466880 \pm$ \\
& $0.566923 \mathrm{i}\}$ & $-8.699320 \times 10^{-4}$, & $0.567469 \mathrm{i}\}$ & $0.567469 \mathrm{i}\}$ \\
\hline \multirow{3}{*}{ 200-th } & $\left\{-1.537113+\frac{\pi}{2 \zeta} \mathrm{i}\right.$, & $\left\{-3.715887 \times 10^{-3}\right.$, & $\left\{-1.536740+\frac{\pi}{2 \zeta} \mathrm{i}\right.$, & $\left\{-1.537050+\frac{\pi}{2 \zeta} \mathrm{i}\right.$, \\
& $0.240380+\frac{\pi}{2 \zeta} \mathrm{i}$, & $-7.987464 \times 10^{-4}$, & $0.240603+\frac{\pi}{2 \zeta} \mathrm{i}$, & $0.240295+\frac{\pi}{2 \zeta} \mathrm{i}$, \\
& $1.084164 \pm$ & $6.083815 \times 10^{-5}$, & $1.083662 \pm$ & $1.083357 \pm$ \\
& $0.582417 \mathrm{i}\}$ & $\left.2.426153 \times 10^{-3}\right\}$ & $0.582535 \mathrm{i}\}$ & $0.582535 \mathrm{i}\}$ \\
\hline
\end{tabular}

Table 6: The comparison of the result by the algorithm of [1], and the solutions to higher level Bethe Ansatz equations (7.2) and (7.3) $: N=5, M=4, T=100, \zeta=\frac{\pi}{7}$. States are selected "randomly".

\section{Conclusion}

This work sets the quantum transfer matrix approach to the thermodynamics of spin chains into a rigorous framework which is applicable for sufficiently high temperatures. For temperatures high enough we have proven all those conjectures raised in the literature, whose validity is necessary for a rigorous use of the approach: the exchangeability of the infinite volume and infinite Trotter number limits, the existence of a maximal in modulus Eigenvalue of the quantum transfer matrix which, furthermore, is real and non-degenerate, the well-definiteness of the class of non-linear integral equations describing the Eigenvalues of the quantum transfer matrix along with the existence and uniqueness of their solutions and, finally, the rigorous identification of the non-linear integral equation describing the dominant Eigenvalue of the quantum transfer matrix. In this way, we have rigorously established the integral representation for the per-site free energy of the spin-1/2 XXZ chain which was argued earlier on on the basis of heuristic arguments [5, 21].

We stress that the uniqueness of solutions to the non-linear integral equation established in this work is a rather non-trivial property. While desirable and potentially expected on the basis of physical arguments, examples of non-linear integral equations, seemingly similar to those considered above, are known, for which, on the basis of numerical investigations, uniqueness has been observed to fail [8]. Moreover, another type of nonlinear integral equations directly for the eigenvalues of the quantum transfer matrix [37] is known to have many inequivalent solutions.

While all the exposition of this paper was focused on the spin- $1 / 2 \mathrm{XXZ}$ chain, the reasoning employed in sections 2, 3] is quite general and needs only very weak assumptions. In particular, upon minor modifications, our proof of Theorem 3.2 is applicable to all quantum integrable models associated with a fundamental $R$-matrix.

In this work we have also obtained a characterisation of a subset of subdominant Eigenvalues of the quantum transfer matrix in the high-temperature regime, this after taking the infinite Trotter number limit. As we have shown, the calculation of these Eigenvalues reduces, to leading order in $T^{-1}$, to finding solutions to the Bethe Ansatz equations associated with the ordinary transfer matrix of the spin-1 XXZ chain. The main tool used in this description are the classes $C_{\alpha, \varrho}^{\epsilon}$. The solutions to the Bethe Ansatz equations have been thoroughly studied numerically, and we have found that, for Trotter numbers up to $N=6$, the classes $C_{\alpha, \varrho}^{\epsilon}$ are non-empty. In fact, it appears that, in this range of $N$, they capture around $70 \%$ of all subdominant Eigenvalues for large $T$. For the time being we do not have any further mathematical interpretation of our numerical data. It will be interesting to 
attempt to interpret them on rigorous grounds.

Acknowledgements. The authors would like to thank Patrick Dorey, Andreas Klümper and Eric Vernier for stimulating discussions. The work of FG was supported by the Deutsche Forschungsgemeinschaft within the framework of the research unit FOR 2316. The work of SG and KKK was supported by the CNRS, Projet international de coopération scientifique No. PICS07877: Fonctions de corrélations dynamiques dans la chaîne XXZ à température finie, Allemagne, 2018-2020. JS was supported by JSPS KAKENHI Grants, numbers 15K05208, 18K03452 and $18 \mathrm{H} 01141$.

\section{References}

[1] G. Albertini, S. Dasmahapatra, and B.M. McCoy, "Spectrum and completeness of the integrable 3-state Potts model: A finite size study.", Int. J. Mod. Phys. A7 (1992), 1-53.

[2] H. Bethe, "Zur Theorie der Metalle: Eigenwerte und Eigenfunktionen der linearen Atomkette.", Z. Phys. 71 (1931), 205-226.

[3] F.A. Brézin, G.P. Pohil, and V.M. Finkelberg, "The Schrödinger equation for a system of one-dimensional particles with point interactions.", Viestnik Moskovskogo Universitata 1 (1964), 21-28.

[4] J.-S. Caux and R. Hagemans, "Deformed strings in the Heisenberg model.", J. Phys. A: Math. Theor. 40 (2007), 14605-14647.

[5] C. Destri and H.J. de Vega, "New thermodynamic Bethe Ansatz equations without strings.", Phys. Rev. Lett. 69 (1992), 2313-2317.

[6] _ _ "Unified approach to Thermodynamic Bethe Ansatz and finite size corrections for lattice models and field theories.", Nucl. Phys. B 438 (1995), 413-454.

[7] C. Destri and J.H. Lowenstein, "Analysis of the Bethe Ansatz equations of the chiral invariant Gross-Neveu model.", Nucl. Phys. B 205 (1982), 369-385.

[8] P. Dorey and R. Tateo, "Excited states by analytic continuation of TBA equations.", Nucl. Phys. B 482 (1996), 639-659.

[9] T.C. Dorlas, "Orthogonality and completeness of the Bethe Ansatz eigenstates of the nonlinear Schrödinger model.", Comm. Math. Phys. 154, 2 (1993), 347-376.

[10] T.C. Dorlas, J.T. Lewis, and J.V. Pulé, "The Yang-Yang thermodynamic formalism and large deviations.", Comm. Math. Phys. 124, 3 (1989), 365-402.

[11] F.H.L. Essler, V.E. Korepin and K. Schoutens, "Fine structure of the Bethe Ansatz for the spin- $\frac{1}{2}$ Heisenberg XXX model.", J. Phys. A: Math. Gen. 25, (1992), 4115-4126.

[12] M. Dugave, F. Göhmann, and K.K. Kozlowski, "Thermal form factors of the XXZ chain and the largedistance asymptotics of its temperature dependent correlation functions.", J. Stat. Mech. 1307 (2013), $\mathrm{P} 07010$.

[13] _ , "Low-temperature large-distance asymptotics of the transversal two-point functions of the XXZ chain.", J. Stat. Mech. 1404 (2014), P04012. 
[14] M. Dugave, F. Göhmann, K.K. Kozlowski, and J. Suzuki, "Thermal form factor approach to the ground-state correlation functions of the XXZ chain in the antiferromagnetic massive regime.", J. Phys. A: Math. Theor. P. Kulish memorial special issue 49 (2016), 394001.

[15] L.D. Faddeev, E.K. Sklyanin, and L.A. Takhtadzhan, "The quantum inverse scattering method.", Teor. Math. Phys. 40 (1979), 194.

[16] M. Gaudin, "Thermodynamics of a Heisenberg-Ising ring for $\Delta \geq 1$.", Phys. Rev. Lett. 26 (1971), 13011304.

[17] F. Göhmann, M. Karbach, A. Klümper, K.K. Kozlowski, and J. Suzuki, "Thermal form-factor approach to dynamical correlation functions of integrable lattice models.", J. Stat. Mech. (2017), 113106, cond-mat.statmech: 1708.04062 .

[18] F. Göhmann, A. Klümper, and A. Seel, "Integral representations for correlation functions of the XXZ chain at finite temperature.", J. Phys. A: Math. Gen. 37 (2004), 7625-7652.

[19] __ , "Integral representations for the density matrix of the XXZ chain at finite temperatures.", J. Phys. A: Math. Gen. 38 (2005), 1833-1842.

[20] A. Klümper, "Free energy and correlation lengths of quantum chains related to restricted solid-on-solid lattice models.", Ann. der Physik 1 (1992), 540-553.

[21] _ _ "Thermodynamics of the anisotropic spin-1/2 Heisenberg chain and related quantum chains. ", Z. Phys. B: Cond. Mat. 91 (1993), 507-519.

[22] T. Koma, "Thermal Bethe-Ansatz method for the one-dimensional Heisenberg model.", Prog. Theor. Phys. 78 (1987), 1213-1218.

[23] _ , "Thermal Bethe-Ansatz method for the spin-1/2 XXZ Heisenberg model.", Prog. Theor. Phys. 81 (1989), 783-809.

[24] J.T. Lewis, J. V. Pulé, and V.A. Zagrebnov, "The large deviation principle for the Kac distribution.", Helv. Phys. Acta 61 (1988), 1063-1078.

[25] E.H. Lieb and W. Liniger, "Exact analysis of an interacting Bose gas. I. The general solution and the ground state.", Phys. Rev. 130 (1963), 1605-1616.

[26] E. Mukhin, V. Tarasov, and A. Varchenko, "Bethe algebra of homogeneous XXX Heisenberg model has simple spectrum.", Comm. Math. Phys. 288, 1 (2009), 1-42.

[27] O. Rojas, S.M. de Souza, and M.T. Thomasz, "High temperature expansion for a chain model.", J. Math. Phys. 43 (2002), 1390-1407.

[28] D. Ruelle, "Statistical mechanics: rigorous results", W.A. Benjamin, Inc., 1969.

[29] M. Shiroishi and M. Takahashi, "Integral equation generates high temperature expansion of the Heisenberg chain.", Phys. Rev. Lett. 89 (2002), 117201.

[30] K. Sogo, "Ground state and low-lying excitations in the Heisenberg XXZ chain of arbitrary spin S.", Phys. Lett. 104 A (1984), 51-54. 
[31] K. Sogo and M. Wadati, "Quantum inverse scattering method and Yang-Baxter relation for integrable spin systems.", Prog. Theor. Phys. 68 (1982), 85-97.

[32] J. Suzuki, Y. Akutsu, and M. Wadati, "A new approach to quantum spin chains at finite temperature.", J. Phys. Soc. Japn. 59 (1990), 2667-2680.

[33] M. Suzuki, "Transfer-matrix methods and Monte Carlo simulation in quantum spin systems.", Phys. Rev. B 31 (1985), 2957-2965.

[34] M. Suzuki and M. Inoue, "The ST-Transformation Approach to Analytic Solutions of Quantum Systems. I: General Formulations and Basic Limit Theorems.", Prog. Theor. Phys. 78 (1987).

[35] M. Takahashi, "One-dimensional Heisenberg model at finite temperature.", Prog. Theor. Phys. 42 (1971), 1289.

[36] _ "Correlation length and free energy of the $S=1 / 2$ XXZ chain in a magnetic field.", Phys. Rev. B 44 (1991), 12382-12394.

[37] M. Takahashi, Simplification of thermodynamic Bethe Ansatz equations, Physics and Combinatorics, Edts. A.K. Kirillov and N. Liskova, World Scientific, Singapore, (2001), 299-304.

[38] C.N. Yang and C.P. Yang, "Thermodynamics of a one-dimensional system of bosons with repulsive deltainteractions.", J. Math. Phys. 10 (1969), 1115-1122. 